\title{
Violence in South African adolescents
}

Citation for published version (APA):

Sui, X. (2020). Violence in South African adolescents: a road to recovery. [Doctoral Thesis, Maastricht University]. Maastricht University. https://doi.org/10.26481/dis.20201124xs

Document status and date:

Published: 01/01/2020

DOI:

10.26481/dis.20201124xs

Document Version:

Publisher's PDF, also known as Version of record

\section{Please check the document version of this publication:}

- A submitted manuscript is the version of the article upon submission and before peer-review. There can be important differences between the submitted version and the official published version of record.

People interested in the research are advised to contact the author for the final version of the publication, or visit the DOI to the publisher's website.

- The final author version and the galley proof are versions of the publication after peer review.

- The final published version features the final layout of the paper including the volume, issue and page numbers.

Link to publication

\footnotetext{
General rights rights.

- You may freely distribute the URL identifying the publication in the public portal. please follow below link for the End User Agreement:

www.umlib.nl/taverne-license

Take down policy

If you believe that this document breaches copyright please contact us at:

repository@maastrichtuniversity.nl

providing details and we will investigate your claim.
}

Copyright and moral rights for the publications made accessible in the public portal are retained by the authors and/or other copyright owners and it is a condition of accessing publications that users recognise and abide by the legal requirements associated with these

- Users may download and print one copy of any publication from the public portal for the purpose of private study or research.

- You may not further distribute the material or use it for any profit-making activity or commercial gain

If the publication is distributed under the terms of Article $25 \mathrm{fa}$ of the Dutch Copyright Act, indicated by the "Taverne" license above, 


\section{Violence in South African Adolescents:}

A Road to Recovery

Xincheng (Alicia) Sui 


\title{
Violence in South African Adolescents:
}

A Road to Recovery

\author{
DISSERTATION \\ to obtain the degree of Doctor at Maastricht University, \\ on the authority of the Rector Magnificus, Prof.dr. Rianne M. Letschert, \\ in accordance with the decision of the Board of Deans, \\ to be defended in public \\ on Tuesday 24 November 2020, at 13:00 hours
}

by

Xincheng Sui

(C) Copyright Xincheng Sui, Maastricht University, 2020

ISBN/EAN: 978-90-830912-7-3

Cover design by: Xincheng Sui

Printed by: Proefschriftenprinten.nl

All rights are reserved. No part of this book may be reproduced, distributed, or transmitted

in any form or by any means, without prior written permission of the author. 


\section{Supervisors:}

Prof. dr. Robert A. C. Ruiter (Maastricht University, the Netherlands)

Prof. dr. Priscilla Reddy (Human Sciences Research Council, South Africa)

\section{Co-supervisor:}

Dr. Karlijn Massar (Maastricht University, the Netherlands)

\section{Assessment committee}

Prof. dr. Gerjo Kok (Chair)

Prof. dr. Catrin Finkenauer (Utrecht University, the Netherlands)

Prof. dr. Relebohile Moletsane (University of Kwazulu-Natal, South Africa)

Prof. dr. Rik Crutzen

Prof. dr. Kai Jonas

\section{Contents}

Chapter 1. General introduction

Chapter 2. Violence typologies and sociodemographic correlates in South African adolescents: a three-wave cross-sectional study

Chapter 3. The associations of internal and external protective factors with violence victimisation and violence perpetration in South African adolescents

Chapter 4. Violence exposure in South African adolescents: differential and cumulative effects on psychological functioning

Chapter 5. Exposure to violence across multiple contexts and health risk behaviours in South African adolescents: the moderating role of emotion dysregulation

Chapter 6. Developmental assets in South African adolescents exposed to violence: a qualitative study on resilience

Chapter 7. Factors that enhance resilience in South African adolescents living in high violence communities: a qualitative study on stakeholders' perspectives and their challenges

Chapter 8. General discussion

\section{Reference}

Summary/Samenvatting

Impact

Acknowledgements

Curriculum Vitae 


\section{Chapter 1}

General Introduction 
'South Africa, a country not at war, faces an unprecedented burden of morbidity and mortality arising from violence and injury.' - Seedat et al., 2009.

South Africa is ranked as the third most violent country in the world (Numbeo, 2020). In South Africa, violence is a significant threat to public health (Bowman et al., 2015; Krug \& Dahlberg, 2002; Ward et al., 2012). Violence is defined by the World Health Organisation as "the intentional use of physical force or power, threatened or actual, against oneself, another person, or a group or community, that either results in or has a high likelihood of resulting in injury, death, psychological harm, maldevelopment, or deprivation" (Krug \& Dahlberg, 2002 , p. 5). Nearly half of South Africa's injury-related deaths are due to violence, and the mortality rate from interpersonal violence $(64.8$ per 100,000$)$ is seven times higher than the global rate (Norman, Matzopoulos, Groenewald, \& Bradshaw, 2007). The epidemic of violence in South Africa costs the country over R28 billion per annum, amounting to $1 \%$ of the GDP (KPMG, 2014).

The high prevalence of violence is entwined with the persistent rates of interpersonal crimes in the country, particularly murder, sexual offences, robbery, and assault with the intention to inflict grievous bodily harm (Urban Safety Reference Group, 2019). It is established that most types of violence stem from the extreme inequality in South Africa. The country has a Gini coefficient of 0.63 , indicating a high degree of income inequality (The World Bank, 2019). Indeed, violence is fueled by social and structural determinants, including radicalised poverty, persistent unemployment, extreme income inequality, patriarchal notions of masculinity that promotes male dominance, extensive exposure to abuse in childhood, access to firearms, excessive substance misuse, and weaknesses in law enforcement in response to violence (Seedat et al., 2014). Since South Africa is a fledgling democracy following the end of Apartheid in 1994, and is currently undergoing political and socio-economic transition, it is important to further the empirical work to address the critical problem of violence and to improve the quality of life and subjective well-being of South Africans. Therefore, this dissertation seeks to enhance the understanding of violence, its negative impact on people, as well as the opportunities to sustain well-being living in the violence-prone country.

\section{Context and Core Concepts}

\section{Adolescents' Risk for Maladjustment}

Adolescents in South Africa disproportionately suffer the consequences of violence (Centers for Disease Control and Prevention [CDC], 2009), which is in part due to their developmental phase. More specifically, adolescents are in a developmental stage characterised by multiple and rapid biological, emotional, and social changes and are particularly prone to maladjustment (Eisman, Stoddard, Heinze, Caldwell, \& Zimmerman, 2015). During this stage, they have greater sensitivity to environmental stressors and are at higher risk for the development of psychological problems (Botticello, 2009; Casey et al., 2010). In addition, adolescents are sensation- and novelty-seeking, and thus have a propensity for aggression and violence, as well as behaviours that put them at risk for negative health outcomes, such as smoking, substance use, and risky sexual behaviours (Reyna \& Farley, 2006).

The transition from youth to healthy adulthood is largely dependent on the environment in which young people live, and the community and social structure that they are exposed to (Reddy et al., 2011). Due to the already stressful developmental transition of adolescents, violence exposure can exacerbate maladjustment, putting them at higher risk for internalising symptoms (e.g., depression, anxiety, posttraumatic stress) and externalising symptoms (e.g., aggression, substance use) (Finkelhor, Ormrod, \& Turner, 2007; Mrug \& Windle, 2010). Moreover, violence exposure can foster health risk behaviours in adolescents, and these behaviours tend to cluster and the majority of adolescents engage in multiple risk behaviours that hamper their physical health (e.g., both alcohol and drug use; Bobrowski, Czabala, \& Brykczynska, 2007; Coleman, Wileyto, Lenhart, \& Patterson, 2014; MacArthur et al., 2012). The psychological and behavioural maladjustments experienced in adolescents, if not prevented or treated, can have a significant impact on their academic and economic outcomes, and psycho-social functioning and (physical) health in adulthood (Botticello, 2009; Das-Munshi et al., 2016). Moreover, violence exposure often increases from childhood into adolescence. A longitudinal study of a cohort of South Africans from birth to age 20 showed that only $1 \%$ of the sample had not been exposed to or experienced violence in their home, school and/or community in these two decades (Richter, Mathews, Kagura, \& Nonterah, 2018). On average, the adolescent group makes up $18.5 \%$ of the total population in South Africa (Statistics South Africa, 2016). Given the sheer size of this population, the high prevalence of violence victimisation in adolescents, and the short- and long-term implications of violence on their psychological and behavioural functioning, it is crucial to enhance the understanding of violence in this vulnerable group to inform the development of targeted and tailored interventions. 


\section{Violence Victimisation in Adolescents}

Violence can be experienced directly, through personal experience of intentional acts perpetuated by another person to cause harm, or indirectly, through witnessing or hearing violent episodes in one's social surroundings (Sui et al., 2018). Due to the high prevalence of violent crimes in South Africa, it is not surprising that adolescents are commonly exposed to violence in their communities, such as being threatened or attacked by someone with a knife and hearing gun shots (Kaminer, du Plessis, Hardy, \& Benjamin, 2013). Adolescents may also be exposed to violence in more proximal environments, such as at home and in school. For example, research has shown that witnessing parents physically fight is prevalent (Richter et al., 2018), and is reflected in the high rates of violence against women in South Africa. It was documented that $15.2 \%$ of women living with a partner were physically abused in the 12 months preceding the South African Demographic and Health Survey in 2016 (Statistics South Africa, 2016). Furthermore, the results of the 2012 National Schools Violence Study in South Africa showed that one in five adolescents had been a victim of violence in school. More specifically, $12.2 \%$ of learners had been threatened with violence by someone at school, $6.3 \%$ had been physically assaulted, $4.7 \%$ had been sexually assaulted, and $4.5 \%$ had been robbed at school (Burton \& Lezanne, 2013). In addition, it is 'normal' for South African adolescents to be exposed to multiple types of violence across different developmental contexts (Richter et al., 2018), i.e., poly-victimisation (Finkelhor et al., 2007). A recent study indicates that the majority of South African adolescents had experienced two or more types of victimisation and over a third had experienced five or more types of victimisation (Leoschut \& Kafaar, 2017). This means that many South African adolescents have few safe areas at home, at school, and in the community.

\section{Violence Perpetration in Adolescents}

Not only are adolescents at risk for violence victimisation, they may also be the perpetrators of violence. Youth involvement in violence disproportionately affects low- and middleincome countries (MacDonald et al., 2009). Furthermore, the rate of violence perpetration increases throughout the developmental period, and $89 \%$ of South African adolescents were reported to be aggressive by fighting with and bullying peers (Richter et al., 2018). Experiencing one or more risk factors, such as exposure to forms of physical and sexual abuse, marihuana use, and alcohol abuse during adolescence, increases the likelihood of the perpetration of violence, aggression and crime as adults, irrespective of an underlying mental illness (Mitjans et al., 2019).

In a developing country like South Africa, adolescents are particularly at risk for violent behaviours as they are living in a challenging environment that perpetuates the use of violence. Studies have shown that experiencing both indirect and direct victimisation, across adolescents' developmental contexts, is associated with aggressive behaviour and delinquency in later life, presumably because these young people are repeating the violence they experienced (Jeong, Davis, Han, 2015; Liu, Mumford, \& Taylor, 2018; Voisin \& Hong, 2012). Compounding this issue are the single-parent households that are common in South Africa and are characterised by limited care, lack of nurturing parenting and adequate supervision, which have been associated with violence perpetration and incarceration in adolescents (Dekel, Abrahams, \& Andipatin, 2018; Harper and McLanahan, 2004). In addition, involvement in gang activities and the easy access to weapons and intoxicating substances in many South African communities are also risk factors that promote violence perpetration at a young age (Reddy et al., 2013).

\section{Adolescents and Resilience}

Exposure to violence can hamper positive developmental outcomes in adolescents, putting them at risk for psychological problems, health risk behaviours, and violence perpetration (Finkelhor, Ormrod, \& Turner, 2007; Mrug \& Windle, 2010). However, not all youth who are exposed to violence experience these negative developmental outcomes (Ward, Martin, Theron, Distiller, 2007; Ostaszewski \& Zimmerman, 2006), which suggests a variation in adolescents' resilience to the negative impact of violence. Resilience is the successful coping with, or the overcoming of, risk and adversity, and the development of competence in the face of severe stress and hardship (McKnight \& Loper, 2002). A key requirement of resilience is the presence of both risk exposure and protective factors that either help bring about a positive outcome, or reduce or avoid a negative outcome (Fergus \& Zimmerman, 2005). Therefore, it is particularly relevant to understand adolescent resilience in the presence of a South African context where violence and crime are highly prevalent.

In this dissertation, resilience is not operationalised as a static trait of an adolescent, but rather as a process that involves an interplay of protective factors in multiple domains of young people's lives that enhance positive development, in spite of the presence of violence. The individual and environmental protective factors that a young person needs to attain positive developmental outcomes are known as developmental assets (Scales, Benson, Leffert, \& Blyth, 2000; Scales, Benson, Roehlkepartain, Semsa, \& Van Dulmen, 2006; Jain, Buka, Subramanian, \& Molnar, 2012). Building skills and enhancing the external resources of adolescents, such as social support, may activate the process in which they are able to utilise strengths from one area to recover in other affected domains in the face of adversity (Harvey, 2007). It is crucial to note that South African adolescents are exposed to violence across multiple developmental contexts, putting them at risk for relatively few safe places, and a possible compromised social support system. Therefore, more so than the young people living in more predictable and less harsh circumstances, South African adolescents are particularly in need to discover and enhance their internal and external developmental assets. Thus, understanding the protective factors that positively contribute to resilient 
developmental outcomes may aid in the development of effective and lasting solutions for adolescents to flourish in life and help inform strengths-based interventions. Focusing on resilience - and thereby on intrinsic strengths rather than weaknesses - may also prevent the negative labelling of victims of violence and further alienating the vulnerable group of adolescents.

\section{Gaps in the Current Literature}

Although several national surveys in South Africa have established the magnitude of violence victimisation and violence perpetration among adolescents (e.g. Burton \& Leoschut, 2013; Reddy et al., 2011), more empirical studies are needed to examine the determinants that place young people at risk for becoming either victims or perpetrators of violence. In the literature, violence is often examined as a composite measure, encompassing both indirect and direct victimisation (Chen, 2010; Dubow et al., 2012; Menard, 2002; Moreira, 2008). Some studies only examine a single type of violence (e.g. sexual victimisation, domestic violence), which has the danger to exaggerate the contribution of the particular type of violence to adolescent development, and neglect the differential impact of various types of violence (Finkelhor, Ormrod, Turner, 2007; Turner et al., 2012).

As such, there is a need to a) distinguish the types of violence in research studies, and b) examine them simultaneously, to compare the associations of correlates with different violence variables. This may enhance the understanding of violence and generate tailored and refined recommendations for intervention development. Understanding the complete victim profile of young people, and how the different forms of victimisation they experience intersect, is critical to ensuring that the most vulnerable South Africans are provided with the extensive and targeted interventions required to break free from their heightened vulnerability to victimisation.

Moreover, it is important to examine the associations of both risk and protective factors with violence typologies, as they complement each other and allow for a comprehensive understanding. To date, studies have established the associations of a range of psycho-social risk factors with violence victimisation and violence perpetration, including family and household composition, frequent exposure to violence in the home, harsh and inconsistent parenting, poor parental supervision and monitoring, parental absence due to prolonged illness or hospitalisation, parental substance misuse, unsupportive teachers, living in a disorganised community (Artz et al., 2016), being a male (Begle et al., 2011; Moreira et al., 2008), substance use (Mrug \& Windle, 2009), dysfunctional family (Schiff, Pat-Horenczyk, Ziv, \& Brom, 2017), unsupportive teachers (Marachi, Avi Astor, \& Benbenishty, 2007), and access to firearms and intoxicating substances in communities (Seedat et al., 2009). These studies are important and consist of the majority of research in youth violence literature. However, only focusing on risk factors may prevent shedding light on the strengths of adolescents that could become the focus of preventive interventions, and these studies also neglect the fact that many who are exposed to violence do overcome adversities and cope successfully, and grow up to be adjusted adults (Ward et al., 2007). Furthermore, research has only recently focused on the understanding of resilience in the context of violence exposure (Jain \& Cohen, 2013; Ward, Martin, Theron, Distiller, 2007). Resilience research is also somewhat limited because it typically includes a single protective factor in the studies (Fergus \& Zimmerman, 2005). To fill part of these gaps in the literature, part of the objectives in this current dissertation include the understanding of the protective factors that are associated with different violence typologies, as well as the protective factors that can enhance resilience in adolescents at risk for violence exposure. This will allow us to gain an in-depth understanding of the nuances of the processes that lead to resilient developmental outcomes, which can in turn be used to design intervention efforts.

\section{Project Overview}

At its core, this dissertation forms a needs assessment to understand the problem of violence in South African adolescents. The objectives of the dissertation are three-fold:

1) To examine the associations of sociodemographic variables and internal and external protective factors with different types of violence victimisation and violence perpetration;

2) To examine the negative impact of different types of violence victimisation on adolescents psychological functioning and health risk behaviours; and

3) To explore the protective factors that enhance resilient developmental outcomes in adolescents at risk for violence victimisation.

Both quantitative and qualitative methods were employed to ensure that ultimately the results in this dissertation can inform targeted and tailored interventions, to reduce the risk for violence victimisation and violence perpetration in adolescents, and promote the strengthening of developmental assets to overcome the negative impact of violence on adolescents' development. 
Two theoretical frameworks were used to lay the foundation of this dissertation to obtain a holistic understanding of research objectives. First, the Ecological Systems Theory (Bronfenbrenner, 1979) suggests that a developing individual is situated within layers of ecological systems, namely, micro-, meso-, exo-, macro-, and chronosystems. An individual's microsystem consists of the immediate environments of home, school, peers, and community. The mesosystem comprises interactions of these immediate environments. The exosystem is the set of indirect environments that may still have an impact on adolescents' lives, such as extended family, parents' workplaces, and social services in the communities. One's macrosystem represents the external influences on individuals, such as cultural, social, historical, and political influences. Lastly, the chronosystem refers to the changes over time in these ecosystems. The interactions across the ecological systems as well as the interactions between different systems and the adolescent shape their development and adaptation.

Second, the Developmental Assets Framework considers that assets that reflect one's core developmental processes are operated at multiple ecological levels (Scales \& Leffert, 1999). A distinction is made between internal and external assets. Commitment to learning, positive values, social competencies, and positive identity are internal assets that youth develop. Support, empowerment, boundaries and expectations, and constructive use of time are the external assets that are provided to youth by parents, school, peers, and community (Scales, Benson, Leffert, \& Blyth, 2000). In light of these two guiding theories, a socialecological approach was used for the needs assessment in this dissertation to ensure that the results are comprehensive and contextually relevant to adolescents living in high-violence communities.

\section{Outline of the Chapters}

Chapter 2 examined the prevalence of different types of violence victimisation and violence perpetration, as well as their sociodemographic correlates in three nationally representative samples of South African adolescents $(n=30,007)$. This study painted an overview of the problem of violence among adolescents in the country between 2002 and 2011.

Chapter 3 examined the associations of internal and external protective factors with different types of violence victimisation and violence perpetration. Specifically, a survey was administered to a sample of adolescents $(n=435)$ living in high-violence communities in Cape Town, South Africa. This study aimed to provide a comprehensive understanding of the protective factors associated with victimisation and perpetration experiences in adolescents from an ecological perspective.
Chapter 4 examined the psychological consequences of violence victimisation in a representative sample of South African adolescents in the Western Cape Province $(n=1574)$. Both differential and cumulative effects of indirect and direct violence exposure across multiple contexts on psychological functioning - e.g. hopelessness, anxiety, depression, perceived stress, and suicidal ideation - were investigated.

Chapter 5 examined the effects of different types of violence victimisation on a composite measure of health risk behaviours in a representative sample of South African adolescents $(n=925)$ in the Western Cape Province and the moderating role of emotion dysregulation. In addition, the role of gender in these associations was investigated.

Chapter 6 explored the internal and external factors that positively enhance resilience in a sample of Cape Town adolescents who had exposure to violence $(n=30)$. Utilising individual semi-structured interviews, this study obtained an in-depth understanding of the protective factors that promote positive psychological, behavioural, and social functioning from the perspectives of adolescents.

Chapter 7 used individual semi-structured interviews to explore parents/caregivers' and school staff's perceptions on the factors that enhance resilience in adolescents living in highviolence communities $(n=36)$. In addition, challenges of these stakeholders associated with responding to the needs of these adolescents were explored. This study complemented the findings in Chapter 6, to enhance the understanding of the protective factors that promote resilience in South African adolescents to the negative impact of violence.

Chapter 8 provided a general discussion of the empirical findings presented in Chapters 2 to 7 of this thesis. The chapter also reflected on the methodology used as well as practical implications. 


\section{Chapter 2}

\section{Violence Typologies and Sociodemographic Correlates in South African Adolescents: A Three-Wave Cross-Sectional Study}




\section{Abstract}

Violence victimisation and violence perpetration may co-occur in adolescents. Understanding the sociodemographic correlates of the independent and joint profiles of victimisation and perpetration may inform preventive interventions. This study examined the associations of sociodemographic factors with four violence typologies, namely, 1) non-involvement in both victimisation and perpetration, 2) victims only, 3) perpetrators only, and 4) victim-perpetrators. Trends in the prevalence of the four violence typologies over the three survey years were also examined. We used data from the three nationally representative South African Youth Risk Behaviour Surveys conducted in 2002, 2008, and 2011 and included a multi-ethnic sample of adolescents $(n=30007$; boy: $46.9 \%$, girls: $53.1 \% ; \mathrm{M}$ age $=16$ years, $\mathrm{SD}=.06)$. The sample consisted of $8030(30.8 \%)$ adolescents who had non-involvement in both victimisation and perpetration, 8217 were victims only (29.8\%), 2504 were perpetrators only (9.0\%), and 7776 were victim-perpetrators $(24.6 \%)$. Logistic regression analyses showed that being a girl increased the odds of non-involvement (OR: 1.47, 99 \% CI: 1.36-1.58) and being victims only (OR: 1.90, $99 \%$ CI: 1.76-2.05). Being a boy increased the odds of being perpetrators only (OR: 0.42, $99 \%$ CI: $0.37-0.47$ ) and victim-perpetrators (OR: 0.51, $99 \%$ CI: 0.47-0.55). Adolescents who did not have an absent mother had higher odds of non-involvement (OR: 0.78, $99 \%$ CI: 0.62-0.97). Lower monthly allowance increased the odds of victimisation only (OR: 0.99, $99 \%$ CI: 0.97-1.00), whereas higher monthly allowance increased the odds of perpetration only (OR: 1.05, 99\% CI: 1.03-1.08). Trend analysis showed that between 2002 to 2011, there was an increase in the prevalence of non-involvement in adolescents $(p<.001)$, a decrease in the prevalence of victims only $(p<.05)$ and victim-perpetrators $(p<.001)$, and no changes in the prevalence of perpetrators only $(p>.05)$. Sociodemographic factors are uniquely associated with different violence typologies, suggesting the need for tailored interventions to target adolescents with differed risks to violence victimisation and perpetration. Strengthening family relations, particularly between mother and child, may protect adolescents from the experiences of victimisation and perpetration.
Globally, violence is a significant public health concern and it is one of the major causes of death among adolescents (Organization, 2019). Violence can be encountered in various ways, such as directly - through personal experiences of victimisation - or indirectly, through witnessing or hearing about violence (Zimmerman \& Posick, 2016). On the other hand, violence can also be perpetrated by intentional uses of physical force to threaten or harm others (Krug, Dahlberg, Mercy, Zwi, \& Lozano, 2002). In South Africa, violence victimisation and perpetration continue to affect the daily lives of adolescents. They are exposed to violence in many or all of the major developmental contexts including home, school, and community (Kaminer, Hardy, Heath, Mosdell, \& Bawa, 2013; Shields, Nadasen, $\&$ Pierce, 2009). Moreover, many adolescents experience poly-victimisation (i.e. exposure to multiple and different types of violence) across these contexts (Kaminer et al., 2013; Leoschut \& Kafaar, 2017; Sui et al., 2018). With respect to perpetration, violent behaviours such as bullying peers, engaging in physical fights, and carrying weapons have persisted in South African adolescents over the past decade (Reddy et al., 2013). Both violence victimisation and perpetration have adverse implications for adolescent developmental outcomes, such as psychological problems and risk behaviours (Shields et al., 2009; Mrug, Loosier, \& Windle, 2008), and importantly, these problems may persist into adulthood, affecting their psychosocial functioning and health (Botticello, 2009; Das-Munshi et al., 2016). Therefore, the current research aims to investigate trends among adolescent violence victimisation and perpetration, and their risk factor correlates to better inform targeted youth interventions in South Africa.

\section{Risk Factors for Victimisation and Perpetration}

Not all adolescents are at risk for violence victimisation and perpetration - one's developmental outcome is an interplay of both individual characteristics and environmental risk factors (Bronfenbrenner, 1979). As such, the understanding of the unique patterns of risk factors that are associated with violence victimisation and perpetration among adolescents may be useful for preventive efforts, as it may help predict the likelihood of the negative outcomes occurring and offer opportunities for interventions to combat such negative consequences. Several sociodemographic factors have been found to put adolescents at risk for violence victimisation and perpetration. For example, older adolescents and ethnic minorities are at higher risk for violence exposure (Finkelhor, Ormrod, \& Turner, 2007; Finkelhor, 2009; Leiner et al., 2014; Sharma \& Marimuthu, 2014). Research showed that in a group of South African adolescents from communities of lower socioeconomic status, the majority have encountered violence at home, in school, and in community (Kaminer et al., 2013). Male adolescents are more likely than their female counterparts to encounter violence victimisation in school and community (Sui et al., 2018), or engage in aggressive behaviours and violence perpetration (Hong, Kim, \& Hunter, 2017; Reingle \& MaldonadoMolina, 2012; Reyes, Foshee, Chen, \& Ennett, 2017). 
Furthermore, adolescents' social context may influence their violence victimisation and perpetration experiences. Specifically, school is one of the major developmental domains where adolescents gather on a regular basis, and this environment can encourage or hamper a range of social and emotional developments (Gevers \& Flisher, 2012). Academic performance in particular reflects an adolescent's ability to learn and their connectedness with the school. Research found that lower academic performance, for example lack of commitment to school work, poor study skills, and lower grades are consistently associated with a higher risk for involvement in violence (Bernat, Oakes, Pettingell, \& Resnick, 2012; Henry, Tolan, Gorman-Smith, \& Schoeny, 2012; Herrenkohl, Huang, Tajima, \& Whitney, 2003; van der Merwe, Dawes, \& Ward, 2012) as well as peer victimisation (Hammig \& Jozkowski, 2013).

Moreover, one's family structure, particularly residing in a single-parent household is significantly associated with both violence victimisation and perpetration in youth (Finkelhor et al., 2007; Hong et al., 2017). In South Africa, partly due to the HIV/AIDS epidemic, approximately 3.5 million children are orphaned (Meintjes, Hall, Marera, \& Boulle, 2010). One review showed that orphans in sub-Saharan Africa living in extended families are particularly vulnerable to psychological, sexual, and physical abuse (Morantz et al., 2013). Children from families in which one or more parents are absent typically experience stigma, discrimination, and a general lack of social support (Morantz et al., 2013; Raymond \& Zolnikov, 2018). Moreover they have an increased risk for experiencing multiple life adversities and poly-victimisations (Finkelhor et al., 2007; Ellonen \& Salmi, 2011; Turner, Finkelhor, Hamby, \& Henly, 2017, as well as incarceration later on in life (Dekel, Abrahams, \& Andipatin, 2018; Harper \& McLanahan, 2004).

\section{Overlap between Victimisation and Perpetration}

Although some studies have examined violence from the perspectives of victimisation and perpetration separately, and provided insight into the risk factors of these types of violence (Kaminer et al., 2013; Shields et al., 2009; Mrug et al., 2008), it is important to recognise the extent to which adolescents have been both victims and perpetrators of violence. Research has shown that violence victimisation and perpetration are often not distinct experiences and can occur in the same individuals (Reingle \& Maldonado-Molina, 2012; Whiteside et al., 2013). For example, in a sample of South African children, over half of them had experienced all three types of violence, namely, witnessing violence, violence victimisation, and violence perpetration, in both school and community (Shields et al., 2009). Witnessing violence and personal victimisation have also been found to contribute directly to violent behaviours in South African adolescents (Choe, Zimmerman, \& Devnarain, 2012). Moreover, Jeong et al. (2015) found that youth who were victims of school bullying were significantly more likely to have committed a violent offence. Further, there is evidence that violence exposure in childhood (e.g., witnessing parental intimate partner violence, physical abuse) may increase one's risk for bullying perpetration and peer victimisation (Voisin \& Hong, 2012), as well as perpetration of violence towards partners in intimate relationships as adolescents or adults (Liu, Mumford, \& Taylor, 2018; Kaukinen, Buchanan, \& Gover, 2015; Maas, Herrenkohl, \& Sousa, 2008). Therefore, the understanding of violence profiles may be limited if victimisation and perpetration are examined separately, since the victimisation group is likely to contain individuals who are also perpetrators, and vice versa (Kaukinen et al., 2015). This also means that the sociodemographic correlates of victimisation and perpetration among adolescents may not be clearly unravelled and understood without distinguishing the subgroup of victim-perpetrators from the sample, as victims only, perpetrators only, and victim-perpetrators may each manifest in a unique interplay of individual and environmental factors. For example, a study that examined the independent and joint relationships of victimisation and perpetration in adolescents found that all three violence-affected subgroups were associated with elevated levels of risk factors than the adolescents who had neither victimisation nor perpetration experiences. Furthermore, there are variations in the risk profiles across the violence subgroups as victim-perpetrators demonstrated the greatest psychosocial impairment, such as lower family finances, single parent family, higher number of life stressors, and higher levels of risk behaviours (Russell, Nurius, Herting, Walsh, \& Thompson, 2010).

In addition, studies found that adolescents who are both victims and perpetrators of violence are at higher risk for adverse developmental outcomes, such as socially deviant behaviours, peer rejection (Georgiou \& Stavrinides, 2008), depression, and alcohol and drug use (Haynie et al., 2013). These findings suggest that victim-perpetrators are especially vulnerable to maladjustment, pointing to the importance to include this subgroup of adolescents in research to discern commonalities and distinctions in the associated correlates in comparison with other violence typologies.

\section{This Study}

Although violence is recognised as a significant public health concern in South Africa, the prevalence of youth violence victimisation, violence perpetration, and the overlap of the two have received little attention. One aim of the current research is to examine the psychosocial correlates associated with different subgroups of adolescents affected by violence, by considering both the distinctive and joint nature of victimisation and perpetration. Specifically, we consider the four violence typologies that have been consistently documented in the literature, namely, 1) neither victimisation nor perpetration histories, 2) victimisation histories only, 3) perpetration histories only, and 4) both victimisation and perpetration histories (Leiner et al., 2014; Hong et al., 2017; Reingle \& Maldonado-Molina, 2012). Differentiating violence as such can offer a more comprehensive understanding of 
the unique correlates of each violence typology (Hong et al., 2017; Russell et al., 2010], which in turn enables tailored intervention development. In the current study, we perform secondary data analysis on three cross-sectional surveys that were conducted in a nationally representative sample of South African adolescents in 2002, 2008, and 2011, with the following aims: 1) to examine the prevalence of the four different violence typologies and their sociodemographic correlates at each survey time point; 2) to examine the associations of demographics, academic performance, and parental absence with each violence typology; 3 ) to establish trend changes in the prevalence of the four violence typologies from 2002 to 2011 .

\section{Methods}

\section{Participants}

This study used secondary data from three cross-sectional South African Youth Risk Behaviour Surveys (YRBS) conducted in 2002, 2008, and 2011 (Reddy et al., 2013; Reddy et al., 2003; Reddy et al., 2010). Each survey involved a nationally representative and multiethnic (black, white, coloured, and Indian) sample of adolescents from grade 8 to 11 in public secondary schools. Only the participants between the ages of 11 and 19 years were included in the analysis as this age range indicates adolescence.

\section{Procedure}

A two-stage cluster sampling was used to recruit participants for the surveys. In stage one, schools were stratified based on the country's nine provinces. In each province, twenty-three public schools were selected with a probability proportional to student size, i.e. larger schools have a greater probability to be selected (schools with an enrolment of more than 25 learners per grade were considered large, those with less than 25 learners per grade were small). In stage two, classes from grades 8 to grade 11 were selected using systematic equal probability sampling of classes from each selected school. All learners in the selected classes were eligible to participate and complete the survey in their classrooms. The YRBS measured sociodemographics characters and different types of risk behaviours in adolescents, including behaviours related to infectious disease (e.g. sexual activity), chronic disease (e.g. physical activity), injury and trauma (e.g. violence), and mental health (e.g. substance use). For the purpose of this study, we extracted data on participants' sociodemographic characteristics and behaviours related to injury and trauma, specifically, exposure to violence victimisation and involvement in violence perpetration.

\section{Measures \\ Sociodemographics}

Data were collected on participants' gender $(1=$ girl, $0=$ boy), age, grade (grade 8 to 11$)$, race (black, coloured ${ }^{1}$, Indian, white, other), monthly allowance ${ }^{2}$ ('don't get any spending money' to 'more than R50'), academic performance ('mostly F's' to 'mostly A's'), and parental absence ('I don't have a father (mother)/my father (mother) is dead'). Parental absence was further coded into three binary variables $(1=$ father absence, mother absence, or both parents absence, $0=$ no absence). The higher scores on the scale variables indicate higher levels of the construct, except for the binary variables of gender and parental absence. Dummy variables were created for race. The majority of adolescents indicated themselves as black and were treated as the reference group in the analyses.

\section{Violence Victimisation}

Participants reported their experiences on six types of indirect and direct victimisation:

Feeling unsafe in and around school. Participants were asked to indicate how often they missed school in the past 30 days because they felt they would be unsafe in school, and because they felt they would be unsafe on their way to and from school. The responses for these two items were 1 ( 0 days) to 5 ( 6 or more days).

Been threatened by a weapon at school. Participants reported the frequency of being threatened by someone with a gun, knife, panga, or kierrie at school in the past six months. The responses were 1 (Never) to 5 (Very often).

Been bullied. Participants indicated the one way in which they were bullied the most in the past 30 days from the following categories - name calling, physical assault, false rumours, discrimination due to race, discrimination due to religion, sexual jokes/remarks, and others.

Dating violence victimisation. Participants reported whether they have ever been hit, smacked, or physically hurt by boy/girlfriend in the past six months. The responses were 1 (Yes) and 0 (No).

Sexual violence victimisation: participants reported whether they have ever been physically forced to have sex. The responses were 1 (Yes) and 0 (No). 


\section{Violence Perpetration}

Participants reported their experiences on eight types of involvement in violence:

Carry a weapon. Participants reported the frequency of them carrying a weapon, a gun, a knife in the past 30 days. In addition, they were asked to indicate the frequency of carrying a weapon in school in the past 30 days. The responses for these four items were 1 (Never) to 5 (Very often).

Threaten others with a weapon. Participants reported the frequency of them threatening someone with a weapon in school in the past six months. The responses were 1 (Never) to 5 (Very often).

Gang membership. Participants indicated whether they have been a member of a gang in the past six months. The responses were 1 (Yes) and 0 (No)

Dating violence perpetration. Participants reported whether they have ever hit, smacked, or physically hurt their boy/girlfriend in the past six months. The responses were 1 (Yes) and 0 (No).

Sexual violence perpetration. Participants reported whether they have ever physically forced someone to have sex. The responses were 1 (Yes) and 0 (No)

\section{Data Analysis}

Data were analysed using SPSS version 23. To obtain insight into the sample characteristics, we first conducted descriptive analyses to investigate the prevalence of each violence typology differentiated by sociodemographic characters for the overall sample and for each survey year. The frequency data were weighted to account for province size and nonresponse. Weights were post-stratified by grade and gender, so that the weighted counts of participants in each grade and gender combination match the provincial population proportions. Participants in highly populated provinces had higher weights than that from less populated provinces to ensure each province was represented equally in the sample. Moreover, intercorrelations were established between all the variables using point-biserial correlation tests at $\alpha=.01$.

Next, binary logistic regression analyses were conducted to examine the associations of sociodemographic factors (gender, age, race, and monthly allowance, academic performance, and parental absence) with each violence typology. Since the regression analyses were done separately for the four violence typologies, we applied Bonferroni correction by using a lower criterion for significance $(\alpha=.01)$ to minimise the potential for Type I error [45].
Lastly, trend analysis of complex survey data was performed to investigate the trends in the four violence typologies across the three different survey years $(2002,2008,2011)$, following the protocol on Conducting Trend Analysis of YRBS data published by the US Centre for Disease Control and Prevention (Centre for Disease Control and Prevention, 2014).

We assigned participants into four distinct groups, namely, non-involvement (NI) in victimisation and perpetration, victim only $(\mathrm{V})$, perpetrator only $(\mathrm{P})$, and victim-perpetrator (VP). To do so, the items for violence victimisation and perpetration were dichotomised, so that 0 reflects the participants who did not experience or involve in the type of victimisation/ perpetration, whereas 1 reflects the ones who did, regardless the frequency of such experience. This resulted in a possible range of $0-6$ for the number of victimisations; and a range of $0-8$ for the number of perpetrations. Participants in the $\mathrm{V}$ group were the ones who had exposure to one or more victimisations, and the ones who perpetrated one or more violence-related behaviours belong to the P group. Participants in NI did not have any victimisation and perpetration histories (i.e., they scored zero for both victimisation and perpetration). Participants who were in the VP group reported at least one exposure to victimisation and at least one violence-related behaviour.

\section{Results}

\section{Violence Typologies: Descriptives}

Table 1 shows the proportions of the adolescents in each violence typology by sociodemographics. The total sample consisted of $n=30007$ adolescents (boy: $46.9 \%$, girls: $53.1 \% ; M$ age $=16$ years, $S D=.06$ ). The resulting violence subgroups for the total sample consisted of adolescents who had non-involvement in both victimisation and perpetration (NI, $n=8030,30.8 \%$ ), victims only (V, $n=8217,29.8 \%$ ), perpetrators only ( $\mathrm{P}, n=2504$, 9.0\%), and victim-perpetrators (VP, $n=7776,24.6 \%$ )

As shown in Table 1, girls disproportionately represented NI (59.0\%) and V groups (65.0\%), whereas boys disproportionately represented P $(67.0 \%)$ and VP groups $(58.3 \%)$. The majority of adolescents in each violence subgroup were black Africans (78.5\% - 84.7\%). The majority of adolescents with absent father were in V group (12.4\%). The majority with absent mother were in VP group (4.1\%), so were the majority with both parents absent $(2.6 \%)$. Adolescents in NI and V groups had the highest academic performance ( $M=4.0$, $S D=0.0)$. Adolescents in the P group had the highest amount of pocket money $(M=3.98$, $S D=0.1)$. 


\section{Associations between Sociodemographics and Violence Typologies}

Table 2 shows the intercorrelations between all variables. Being a girl was correlated with NI $(r=.084, p<.01)$ and $\mathrm{V}(r=.146, p<.01)$, whereas being a boy was correlated with $\mathrm{P}(r$ $=-.118, p<.01)$ and $\mathrm{VP}(r=-.150, p<.01)$. Older age was associated with VP $(r=.079, p$ $<.01)$. Black adolescents were associated with VP $(r=.030, p<.01)$. Adolescents of mixed heritage were associated with $\mathrm{V}(r=.029, p<.01)$. Indian $(r=.043, p<.01)$ and white adolescents $(r=.021, p<.01)$ were associated with NI; they were also associated with $\mathrm{P}$ $(r=.051, p<.01 ; r=.024, p<.01$, respectively). Father absence was associated with $\mathrm{V}$ $(r=.021, p<.01)$. Not having absent mother $(r=-.016, p<.01)$ was associated with NI, whereas mother absence was associated with VP $(r=.020, p<.01)$. Both parents absence was associated with VP $(r=.017, p<.01)$. Higher academic performance was associated with NI $(r=.017, p<.01)$. Higher monthly allowance was associated with NI $(r=.015, p$ $<.01)$ and $\mathrm{P}(r=.044, p<.01)$, whereas lower monthly allowance was associated with VP $(r=-.018, p<.01)$.

\section{Binary Logistic Regression Analyses}

Significant associations of sociodemographic variables with the four violence typologies are reported here. A full overview of the results is presented in Table 3.

Non-involvement. Being a girl (OR: 1.47, $99 \%$ CI: 1.36-1.58) and having a younger age (OR: 0.94, 99 \% CI: 0.91-0.96) were associated with higher odds of NI. Black adolescents had higher odds of NI than adolescents of mixed heritage (OR: 0.82, $99 \% \mathrm{CI}: 0.73-0.92$ ). Further, Indian (OR: 1.49, $99 \%$ CI: 1.27-1.75) and white adolescents (OR: 1.22, $99 \%$ CI: 1.05-1.42) had higher odds of NI than black adolescents. In addition, adolescents who did not have an absent mother had higher odds of NI (OR: 0.78, $99 \%$ CI: 0.62-0.97).

Victims only. Being a girl (OR: 1.90, $99 \%$ CI: 1.76-2.05) was associated with higher odds of only being a victim. Adolescents of mixed heritage had higher odds of being victimised only than black adolescents (OR: 1.22, $99 \%$ CI: 1.09-1.37). Black adolescents had higher odds of being victimised only than Indian adolescents (OR: 0.80, $99 \%$ CI: 0.67-0.96). Adolescents who had lower amount of monthly allowance had higher odds of being victimised only (OR: 0.99, 99 \% CI: 0.97-1.00).

Perpetrators only. Being a boy (OR: 0.42, $99 \% \mathrm{CI}$ : 0.37-0.47) was associated with higher odds of only being a perpetrator of violence. Indian (OR: 1.70, $99 \%$ CI: 1.35-2.14) and white adolescents (OR: 1.24, 99 \% CI: 0.99-1.55) had higher odds of only perpetrating violence than black adolescents. Higher monthly allowance was associated with higher odds of only perpetrating violence (OR: $1.05,99 \%$ CI: 1.03-1.08).
Victim-perpetrators. Being a boy (OR: 0.51, $99 \%$ CI: $0.47-0.55)$ and having an older age (OR: 1.10, 99 \% CI: 1.07-1.13) were associated with higher odds of being a VP. Black adolescents had higher odds of being VP than Indian adolescents (OR: 0.61, $99 \%$ CI: 0.500.75 ) and white adolescents (OR: 0.67, 99 \% CI: 0.57-0.80). 


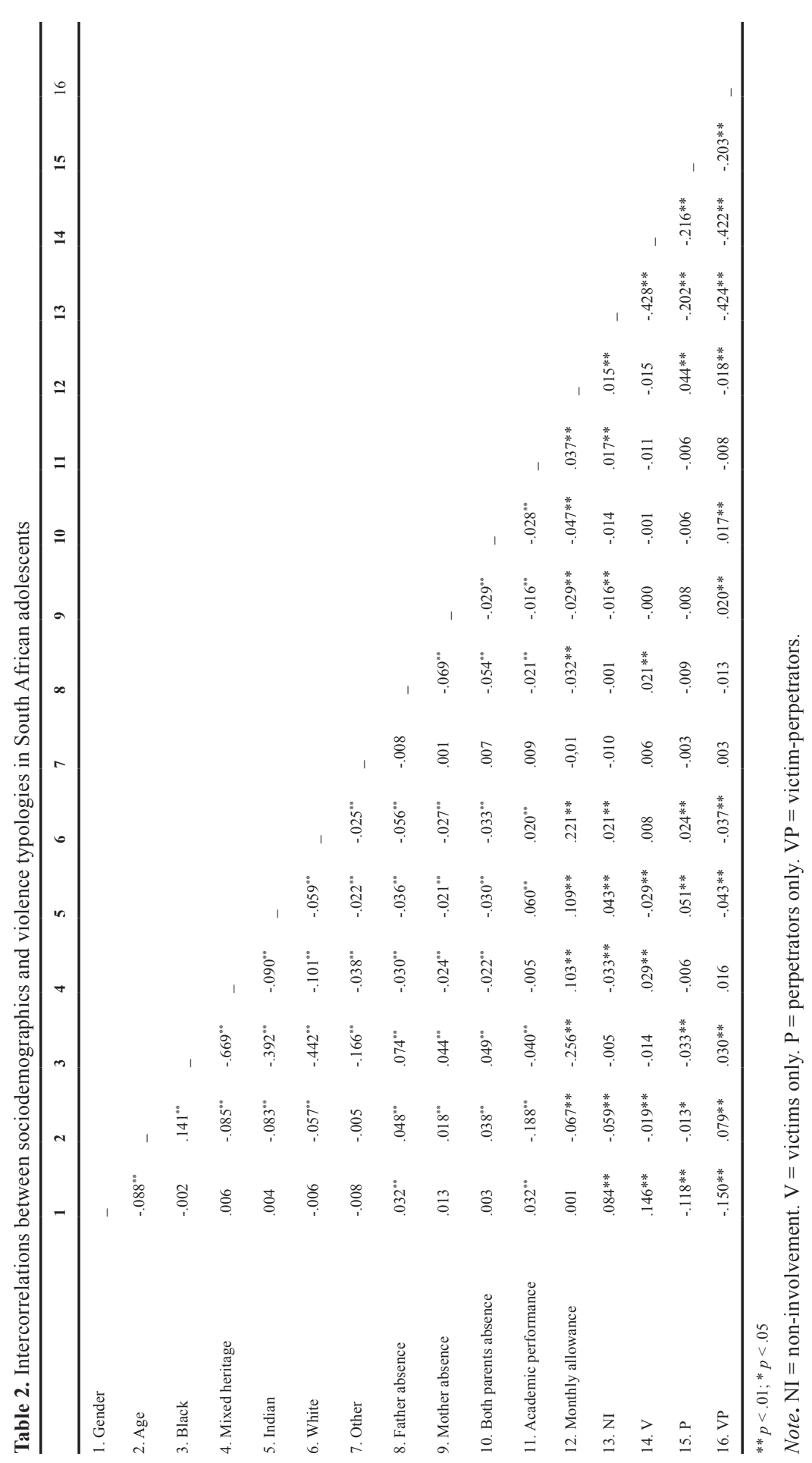


Table 3. Results of binary logistic regression analyses on violence typologies with sociodemographics in South African adolescent

\begin{tabular}{|c|c|c|c|c|c|c|c|c|}
\hline \multirow[b]{2}{*}{ Variables } & \multicolumn{2}{|r|}{ NI } & \multicolumn{2}{|r|}{ v } & \multicolumn{2}{|r|}{$\mathbf{P}$} & \multicolumn{2}{|r|}{ VP } \\
\hline & OR & $99 \%$ CL & OR & $99 \%$ CL & OR & $99 \% \mathrm{CL}$ & OR & $99 \%$ CL \\
\hline Genderabcd & 1,47 & $1.36-1.58$ & 1,90 & $1.76-2.05$ & 0,42 & $0.37-0.47$ & 0,51 & $0.47-0.55$ \\
\hline $\mathrm{Age}^{\mathrm{ad}}$ & 0,94 & $0.91-0.96$ & 0,98 & $0.96-1.01$ & 0,96 & $0.92-1.00$ & 1,10 & $1.07-1.13$ \\
\hline \multicolumn{9}{|l|}{ Black (reference) } \\
\hline Mixed heritage ${ }^{a b}$ & 0,82 & $0.73-0.92$ & 1,22 & $1.09-1.37$ & 0,91 & $0.76-1.10$ & 1,06 & $0.95-1.19$ \\
\hline Indian abced & 1,49 & $1.27-1.75$ & 0,80 & $0.67-0.96$ & 1,70 & $1.35-2.14$ & 0,61 & $0.50-0.75$ \\
\hline White acd & 1,22 & $1.05-1.42$ & 1,13 & $0.97-1.31$ & 1,24 & $0.99-1.55$ & 0,67 & $0.57-0.80$ \\
\hline Other & 0,80 & $0.51-1.25$ & 1,32 & $0.87-2.01$ & 0,87 & $0.42-1.79$ & 0,94 & $0.60-1.47$ \\
\hline Monthly allowance ${ }^{\text {bc }}$ & 1,00 & $0.99-1.02$ & 0,99 & $0.97-1.00$ & 1,05 & $1.03-1.08$ & 1,00 & $0.98-1.01$ \\
\hline Academic performance & 1,01 & $0.98-1.04$ & 0,97 & $0.95-1.00$ & 0,98 & $0.93-1.02$ & 1,02 & $0.99-1.05$ \\
\hline Father absence & 1,01 & $0.89-1.14$ & 1,10 & $0.98-1.24$ & 1,00 & $0.82-1.21$ & 0,90 & $0.79-1.02$ \\
\hline Mother absence ${ }^{a}$ & 0,78 & $0.62-0.97$ & 1,07 & $0.87-1.32$ & 0,91 & $0.64-1.30$ & 1,20 & $0.97-1.47$ \\
\hline Both parents absence & 0,89 & $0.68-1.17$ & 0,88 & $0.67-1.15$ & 1,06 & $0.69-1.62$ & 1,22 & $0.94-1.57$ \\
\hline
\end{tabular}

\section{significant variables for $\mathrm{N}$}

${ }^{\mathrm{b}}$ significant variables for $\mathrm{V}$

${ }^{\circ}$ significant variables for $\mathrm{P}$

¿ significant variables for VP

Note. Significance is at the 0.01 level. $\mathrm{NI}=$ non-involvement. $\mathrm{V}=$ victims only. $\mathrm{P}=$ perpetrators only. $\mathrm{VP}=$ victimperpetrators.

\section{Trend Analysis}

Non-involvement. The overall prevalence of adolescents who had no victimisation and perpetration experiences was $30.8 \%$, (95\% CI: $0.29-0.32)$. The prevalence of these adolescents increased from $24.6 \%$, (95\% CI: $0.23-0.27)$ in 2002 , to $27.5 \%$, (95\% CI: 0.25 $0.30)$ in 2008 , and to $33.1 \%$, (95\% CI: $0.31-0.35)$ in 2011, indicating a significant increasing trend from 2002 to $2011(p<.001)$. Thus, involvement in violence (either victimisation or perpetration) declined over these years.

Victims only. The overall prevalence of adolescents who were victims only and had at least one victimisation experience was $29.8 \%$, $(95 \%$ CI: $0.28-0.31)$. The prevalence of these adolescents decreased from $33.6 \%$ (95\% CI: $0.31-0.36$ ) in 2002, to $28.7 \%$ (95\% CI: $0.27-0.30)$ in 2008 , and to $28.6 \%$ (95\% CI: $0.27-0.30)$ in 2011 , which showed a significant decreasing trend from 2002 to $2011(p<.01)$.

Perpetrators only. The overall prevalence of adolescents who were perpetrators only and perpetrated at least one violence-related act was $9.0 \%$, (95\% CI: $0.08-0.10)$. There were no significant changes in the prevalence of these adolescents across $2002(8.0 \%, 95 \%$ CI: $0.07-0.09), 2008$ (8.9\%, 95\% CI: CI: 0.08-0.10), and 2011 (9.3\%, 95\% CI: 0.09-0.10) $(p>.05)$. Thus, rates of violence perpetration among adolescents aged 11-19 remained relatively stable over these years.

Victim-perpetrators. The overall prevalence of adolescents who had at least one victimisation and perpetration experience was $24.6 \%$, (95\% CI: $0.23-0.26)$. The prevalence of these adolescents decreased from $30.0 \%$, (95\% CI: $0.27-0.34)$ in 2002 and $31.5 \%$, (95\% CI: 0.29 $0.34)$ in 2008 , to $22.5 \%$, (95\% CI: $0.21-0.24)$ in 2011 , which showed a significant decreasing trend from 2002 to $2011(p<.001)$.

\section{Discussion}

This study provided an overview of the national prevalence of the school adolescents in South Africa who were non-involved in both violence victimisation and perpetration, victims only, perpetrators only, and victim-perpetrators in 2002, 2008, and 2011, as well as the trend changes in the prevalence of these four violence typologies across the survey years. Moreover, the associations of demographics, academic performance, pocket money, and parental absence with the violence typologies were examined to understand the unique correlates of each violence subgroup.

Approximately a third of the adolescents in the whole sample were classified as victims only, and another third had non-involvement in both victimisation and perpetration. When the prevalences were examined by each survey year, the proportion of adolescents who were victims only significantly decreased from 2002 to 2011, whereas the adolescents who hadnone-involvement significantly increased from 2002 to 2011. These trend changes reflect a positive social transition in the post-Apartheid era in South Africa, where political violence of discrimination against non-whites (such as forced removal and brutal physical assault) has subsided since the abolishment of Apartheid in 1994. In the years following this event, new legislation and policies have been enforced to prevent crime and improve criminal justice functioning in democratic South Africa (Du Plessis \& Louw, 2005). These changes are likely to have contributed to the overall reduction of violence victimisation among young people.

Moreover, the lowest proportion of adolescents in the whole sample were perpetrators only, with less than 10 percent were found in each survey year and the prevalence remained stable across 2002 to 2011. Importantly, we found that a substantial proportion of adolescents (almost a quarter) were both a victim and a perpetrator of violence. This result is in line 
with other studies (Shields et al., 2009; Whiteside et al., 2013; Choe et al., 2012; Swahn et al., 2012) that demonstrated that victimisation and perpetration experiences can overlap. In South Africa, where high levels of interpersonal violence still exist, such violence is marked by contextual features, such as poverty, unemployment, gender inequality, exposure to abuse in childhood, and access to firearms (Seedat, Van Niekerk, Suffla, \& Ratele, 2014). Both victims and perpetrators may share similar contextual features that put them at risk for both forms of violence. Furthermore, a review has found that exposure to different forms of violence such as sexual, emotional, and physical abuse, and even less severe forms of physical punishment and harsh parenting may have adverse long-term impact, and increase the likelihood of later violence perpetration among youth (Maas et al., 2008). One possible mechanism of such effects may be due to the social learning effects of violence through direct observation and socialisation processes (Bandura, 1977), and that adolescents acquired violent behaviours through learning and internalising it as the "norm" to solve problems, particularly in South Africa where there was a history of violence (van der Merwe et al., 2012). In addition, the risk for violent behaviours can be compounded by the propensity for risk taking behaviours and vulnerability to emotion dysregulation during the developmental phase of adolescence (Bell \& McBride, 2010).

Further, our results showed that each violence typology was associated with different sociodemographic factors. We found that a lower amount of monthly pocket money put adolescents at risk for victimisation. This result corroborates with studies that showed that compared to adolescents from higher socio-economic environments, violence exposure among adolescents from low socioeconomic settings is normative, and they experience violence either directly or indirectly on a daily basis (Kaminer et al., 2013). Conversely, we found that higher amount of pocket money put adolescents at risk for perpetration of violence towards others. This association might be related to the purchase of the highly available alcohol, drugs, and weapons in many South African communities (Burton \& Leoschut, 2013; Matzopoulos, Thompson, \& Myers, 2014), which may in turn promote violent behaviours.

Moreover, we found that boys and girls are at risk for different violence experiences. Being male was a significant predictor for having only perpetrated violence. Adolescents are in a developmental period where they are inclined to be sensation seeking and risk taking (Steinberg, 2007), and boys in particular, are at risk for externalising problems such as aggression, aggressive fantasies, conduct problems, and higher levels of delinquency (Duke, Smith, Oberleitner, Westphal, \& McKee, 2018; Reingle \& Maldonado-Molina, 2012). Our results also indicated that boys were more likely to be victim-perpetrators than girls, which is in line with other studies that showed correlations between violence victimisation and violence perpetration in adolescents (Choe et al., 2012; Jeong et al., 2015). This may be due to the socialisation differences between boys and girls in South Africa, in which boys are more likely to spend time in their neighbourhoods and to be approached by or be involved in gangs (Kaminer et al., 2013; Cooper \& Ward, 2012), putting them at risk for both the exposure to and the conduct of violent activities. Furthermore, boys are more likely than girls to engage in high-risk behaviours such as alcohol and drug use (Croisant, Haque Laz, Rahman, \& Berenson, 2013; Mennis \& Mason, 2012), which can expose them to additional high risk situations, including greater exposure to potential offenders and an elevated risk for both violence victimisation and perpetration (Mrug \& Windle, 2009; Parker \& Bradshaw, 2015).

On the other hand, being a girl was a significant predictor for non-involvement in both victimisation and perpetration. This could be explained in light of findings that girls are more prone to internalising symptoms such as anxiety, depression, and perceived stress during adolescence (Hilt, Cha, \& Nolen-Hoeksema, 2008; Slone \& Mayer, 2015), and may thus experience less tendencies to engage in externalising behaviours such as violence perpetration. Further, girls in our study were also associated with having only victimisation experiences Although some studies have established that boys are at higher risk than girls for victimisation in different contexts such as school and community (Sui et al., 2018; Kaminer et al., 2013) as well as poly-victimisation (Finkelhor, Ormrod, \& Turner, 2007), the current finding indicates the opposite and may highlight the vulnerability of girls to victimisation. According to a national survey in South Africa, females - both girls and adult women - are subjected to high levels of physical violence (Statistical South Africa, 2016) and are more likely to be victimised by certain types of violence than boys, such as sexual assault, rape, and dating violence (Finkelhor, 2009; Duque, Montoya, \& Restrepo, 2011).

Another important finding in our study is that adolescents who did not have an absent mother in the household (absent due to divorce or death) was associated with non-involvement in victimisation and perpetration, suggesting that the presence of maternal support and supervision may have positive implications on an adolescent's life experiences and adjustment. Indeed, functioning parent-child relationship such as family support has been found to be a protective factor that may lower the risk for violence perpetration in boys as well as in adolescents who had exposure to community violence (Massarwi, 2017). Similarly, high mother-child relationship quality such as adequate involvement and supervision, and parenting that satisfies the child's needs for security, exploration, and autonomy, can protect against internalising (e.g. anxiety, depression) and externalising (e.g. aggressive behaviours) adjustment difficulties in youth (Al-Yagon, 2015; Kliewer et al., 2004; McWey, Claridge, Wojciak, \& Lettenberger, 2015; Profe \& Wild, 2017; Villodas, Bagner, \& Thompson, 2018). Conversely, higher levels of maternal rejection are associated with greater behaviour problems such as aggression (Howard, Martin, Berlin, \& Brooks-Gunn, 2011; Schiff, Pat-Horenczyk, Ziv, \& Brom, 2017). Moreover, there is evidence that single fathers have weaker interpersonal and affective bonds with their children, and exhibit less supervision and monitoring (Coles, 2015), 
compounding the possible effects of mother absence. However, the current results prohibit us from drawing firm conclusions, and these interpretations thus remain speculative, and future research is needed to understand the exact underlying processes of family influence on adolescents' experiences of violence victimisation and perpetration.

\section{Limitations and Recommendations}

Although this study provided an overview of the violence trend across three survey years $(2002,2008$, and 2011) among a nationally representative sample of South African adolescents, few limitations should be noted. First, the results of the study are limited to the time period between 2002 and 2011 as a new YRBS has not been conducted in South Africa since 2011. The data consisted of three cross-sectional samples and thus the causal direction of the associations between violence typologies and their sociodemographic correlates cannot be established. Longitudinal studies are necessary to investigate the causality of these associations, as well as the possible moderating variables. Moreover, the intercorrelations between variables are weak in this study. Although they are statistically significant, it may be due to the nature of a large sample and thus may limit the practical implications of these associations. In addition, the reasons for parental absence in life were not explicitly accounted for in this study and require further exploration. For example, a parent may be absent due to work, divorce, or death, and each may have differential impact and underlying mechanisms on adolescents' experiences of violence. Lastly, data were collected in schools and may not be fully representative for adolescents who do not attend school. In South Africa, approximately eight percent of adolescents are secondary school drop-outs (Department of Basic Education, 2011). Due to inadequately developed life and social skills, these adolescents are more likely to engage in high risk activities, including violence (Vayachuta, Ratana-Ubol, \& Soopanyo, 2016). Future research may draw attention on this unique population to obtain a more comprehensive understanding of the violence profiles among different subgroups of adolescents.

\section{Conclusion}

This current study showed a significant reduction in the prevalence of adolescent victims of violence as well as victim-perpetrators between 2002 and 2011 in South Africa, suggesting that the crime and violence reduction strategies implemented in the post-Apartheid era had a positive effect on adolescents' violence victimisation experiences. Our study sheds light on the sociodemographic determinants of violence that are beyond the scope of the police and courts, such as income inequality, ethnicity, gender inequality, and compromised parenting (Seedat et al., 2014). In addition, our results offer insights that presence of mother in an adolescent's life may bring positive influences on the child's development. Interventions may consider strengthening family relations, particularly between mother and child, to protect adolescents from the experiences of violence victimisation and violence perpetration. Since studies have consistently established that family connectedness is associated with positive youth developmental outcomes such as lower levels of violence victimisation and aggression (Al-Yagon, 2015; Kliewer et al., 2004; McWey et al., 2015; Massarwi, 2017), there is an opportunity for families with mother absence to involve extended family members to extend the support and supervision that may be needed to promote safety and positive behavioural adjustment among adolescents. In conclusion, regular monitoring of the prevalence of violence victimisation and violence perpetration as well as the associated correlates may help develop targeted youth interventions in South Africa. Given the heterogeneity of violence profiles and the unique correlates of each violence subgroup as highlighted in our study, one-size-fits-all violence interventions may be ineffective and tailored interventions are needed for adolescents with differed risks to violence exposure and violence perpetration.

\section{List of Abbreviations}

NI: non-involvement (in victimisation and perpetration)

V: victims only

P: perpetrators only

VP: victim-perpetrators

YRBS: Youth Risk Behaviour Survey

\section{Footnotes}

${ }^{1}$ A South African term referred to people of mixed heritage. This term is officially used in statistical publications and census data in South Africa.

${ }^{2}$ The amount of monthly allowance indicated in the three surveys $(2002,2008,2011)$ increased around $30 \%$ over the survey years due to inflation. 


\section{Chapter 3}

The Associations of Internal and External Protective Factors with Violence Victimisation and Violence Perpetration in South African Adolescents 


\section{Abstract}

This study examined the associations of protective factors with different types of violence victimisation and violence perpetration in South African adolescents to inform strengthsbased interventions. Adolescents $(n=435$; boy: $46 \%$, girl: $54 \%$; $M$ age $=16$ years, $S D=$ 1.31) completed a survey that measured individual, peer, family, school, and community protective factors, and their experiences of victimisation (indirect victimisation, crimerelated victimisation, peer victimisation), and perpetration (peer violence perpetration, delinquent behaviours). Results showed that self-confidence, social skills, anger management, emotional insight, and family-, school-, peer-, and community connectedness were associated with less victimisation experiences. Anger management, emotional insight, instrumental coping of emotions of sadness and anger, empathy, and school connectedness were associated with less perpetration experiences. Multi-setting and multi-faceted programmes that include personal skills building and positive involvement of family, peers, teachers, and community stakeholders may be helpful to prevent victimisation and perpetration experiences in adolescents.
Violence exposure is a public health concern and disproportionately affects youth aged 10 to 24 years (World Health Organisation, 2015). In South Africa, violent crime in the forms of murder, rape, robbery are prevalent, contributing to the nation's high rates of injury, morbidity, and mortality (Seedat, Van Niekert, Jewkes, Suffla, \& Ratele, 2009). Within such a violence-prone environment, violence exposure among South African youth has shown to be a norm and a life condition of ongoing threat and danger across multiple developmental contexts (Kaminer, du Plessis, Hardy, \& Benjamin, 2013; Shields, Nadasen, $\&$ Pierce, 2013). A longitudinal study of a cohort of South African youth from birth to age 22 showed that over the past two decades, only less than $1 \%$ of the sample had not been exposed to violence (Richter, Mathews, Kagura, \& Nonterah, 2018). Similarly, other studies have found that the majority of South African adolescents had been exposed to violence directly or indirectly (e.g., witness or hearing about violence) in different contexts, such as witnessing domestic violence at home, having been beaten up in school, and having been threatened to be shot/stabbed in the neighbourhood (Kaminer et al., 2013; Sui et al., 2018). Furthermore, adolescents who are exposed to one form of violence are at increased risk to be exposed to violence in other forms and experience poly-victimisation (Finkelhor, Ormrod, \& Turner, 2007). Poly-victimisation across different developmental contexts is prevalent among South African adolescents and has been found in vast majority of the samples resulting in perceived stress, hopelessness, and suicide ideation (Kaminer et al., 2013; Richter et al., 2018; Sui et al., 2017).

Moreover, not only young people are vulnerable to violence exposure, South Africa also faces a crisis of involvement in violence by young people. A longitudinal study showed trends of increase in violence perpetration over the youth developmental period - in the preschool years, close to half of the sample were reported to be aggressive, starting fights with and bullying other children. In the primary school years, violent behaviour was reported in $65 \%$ of children and increased to $89 \%$ in adolescence (Richter et al., 2018). The South African National Youth Victimisation Study found that almost one in five (18.3\%) of the youth between the age of 12-22 years had considered committing what they knew would be a criminal offence and over half of these young people, not only considering, but had already committed such (Leoschut \& Burton, 2006). In a nationally representative sample in South Africa, behaviours such as bullying peers, engaging in physical fights, and carrying weapons were persistent trends in school learners over the past decade (Reddy et al., 2013).

Violence victimisation and violence perpetration are associated with a range of personal and environmental risk factors, for example, being young males, low socioeconomic status, familial crime, dysfunctional home and school environments, emotion dysregulation, and alcohol abuse and gang membership of young people (Garner \& Hinton, 2010; Hinsberger et al., 2016; Leoschut \& Burton, 2006; Souverein, Ward, Visser, Burton, 2016). In addition, the 
experiences of violence victimisation and perpetration may overlap and occur in the same individuals (Reingle \& Maldonado-Molina, 2012). Youth exposed to violence - whether as victims or as witnesses - are more likely to exhibit aggressive behaviour, such as bullying and fighting in school, and they are at higher risk for delinquency later in life by repeating the violence they experienced (Jeong, Davis, Han, 2015; Liu, Mumford, \& Taylor, 2018). Both exposure to violence and perpetration of violence are associated with youth mental health symptoms (Finkelhor et al., 2007; Mrug \& Windle, 2010; Sui et al., 2018) and health risk behaviours such as smoking, substance use, and risky sexual behaviours (Begle et al., 2011; Sui et al., 2020). These internalising and externalising maladjustment can negatively impact youth education and economic outcomes, psychosocial functioning and health, and criminality in adulthood, leading to a vicious cycle of violence and poor functionality (Richter et al., 2018; Souverein et al., 2016).

While an extensive literature has highlighted the factors that put youth at risk for violence exposure and violence perpetration, more research is needed to enhance the understanding protective factors that help reduce these experiences. Since violence victimisation and perpetration have been identified as serious risk factors for maladjustment, the understanding of the conditions, characteristics, and influences that combat the victimisation and perpetration experiences may complement the existing literature and inform interventions that prevent these adverse experiences and ultimately promote positive youth development and functioning in a context of prevalent crime and violence.

The literature on the factors that positively influence youth development and functioning has placed an emphasis on individual attributes and the presence of ecological resources (Jain, Buka, Subramanian, \& Molnar, 2012; Scales, Benson, Leffert, \& Blyth, 2000). Adolescence is a developmental stage characterised by rapid physiological, cognitive, social, and emotional changes (Crone \& Dahl, 2012). These psycho-social attributes of adolescents play a vital role in the rates of victimisation and perpetration experiences (Pellegrini \& Long, 2002) and are key internal factors that allow them to adapt and overcome the adversities that put them at risk for psychological and behavioural maladjustment. For example, anger management was associated with lower frequencies of physical aggression in a group of African-American adolescents from lower socioeconomic setting (Sullivan, Helms, Kliewer, \& Goodman, 2010). The capacity to control negative emotional arousal may enhance the management of impulsivity and activate adaptive coping processes that refrain adolescents from engaging in risk behaviours (Sui et al., 2020). Another facet of the emotional competency is emotional insight, which is the introspective ability to take time and space to think things through, to work out what was happening and why, and to behave in a manner that is socially appropriate and helpful for oneself in an adverse situation (e.g., self-care) (Gartland, Bond, Olsson, Buzwell, \& Sawyer, 2011). For example, having the knowledge of display rules for sadness has been associated with lower physical victimisation in children. This competency reflects one's understanding of how emotional displays are perceived by others and thus allow young people to adapt and regulate responses in social situations that help them avoid victimisation (Garner \& Hinton, 2010). Moreover, social skills such as cooperation, assertion, responsibility, and self-control were associated with lower likelihood of violent behaviours in physically maltreated children, as these skills allow them to have realistic expectations of social situations and establish effective interpersonal relationships (Holmes, Yoon, Voith, Kobulsky, \& Steigerwald, 2015).

In addition, support from the major developmental contexts such as home, school and community are protective for victimisation and perpetration experiences. For example, close relationship to at least one parent and intensive parental supervision reduced the likelihood of aggression and delinquent behaviours (Lösel \& Farrington, 2012; Kliewer et al., 2004). Perceived parental care was protective for future victimisation experiences in adulthood following sexual abuse in childhood (Scoglio, Kraus, Saczynski, Jooma, \& Molnar, 2019). Teachers' support and attentiveness to incidents of school violence was associated with lower rates of victimisation among learners (Marachi, Astor, \& Benbenishty, 2007). Strong school connectedness and positive school/class climate were associated with less aggression and violent behaviours in adolescents (Lösel \& Farrington, 2012). Peer support buffered the impact of violence exposure on peer-reported aggressive behaviour in adolescents (Benhorin \& McMahon, 2008). Living in a non-deprived and non-violent neighbourhood may prevent youth violence perpetration (Lösel \& Farrington, 2012). These studies suggest that the positive influences from major developmental contexts are crucial to reduce the experiences of victimisation and perpetration.

Furthermore, Life Orientation is a mandatory lesson in South African school education curriculum, where life skills and prosocial behaviours are taught to promote healthy adolescent functioning and adjustment and help learners use their talents to achieve their full physical, intellectual, personal, emotional and social potential (Department of Education, 2011). One of the topics covered in Life Orientation is to build necessary skills and knowledge to refrain from engaging in violent behaviours. Evidence on the effectiveness of this important national education curriculum on youth development is unclear. Yet given the aim of this national education curriculum, it may be helpful to evaluate whether having received Life Orientation lessons is associated with adolescents' lower reports of victimisation and perpetration experiences to inform policy and intervention development.

Taken together, the literature suggests the importance to enhance individual competencies (internal protective factors) and strengthen environmental resources (external protective factors) such as interpersonal support at different ecological contexts, to help adolescents 
avoid violence-encouraging situations and reduce the risk for violence victimisation and perpetration. However, protective factors are not investigated as frequently as risk factors in the literature of youth violence, and the studies that measured protective factors often examined them in associations with single types of violence victimisation or perpetration. Thus, a comparative and comprehensive overview of the protective factors and their associations with different types of violence victimisation and perpetration may be useful.

\section{This Study}

We aimed to address the gaps in the literature by examining the protective factors that are associated with different types of violence exposure and perpetration in a sample of South African adolescents in Cape Town. We included some of the robust factors that have been conceptually and empirically shown to be protective for violence victimisation and perpetration among youth, on the individual, peer, family, school, and community levels. Both internal and external protective factors were included to provide a comprehensive overview of the patterns of these factors in associations with different types of violence.

Moreover, some of the most common types of violence exposure and violence perpetration among South African adolescents were included in this study, such as indirect victimisation, crime-related victimisation, peer victimisation, peer violence perpetration, and delinquent behaviours. The inclusion was informed by the literature and the interviews that we conducted with another sample of South African adolescents on their coping processes following violence exposure (Sui, Massar, Reddy, \& Ruiter, submitted). The results of the current study may provide insights on the developmental implications of adolescents living in a violence-prone environment and help inform targeted interventions to prevent victimisation and perpetration among adolescents.

\section{Methods}

\section{Participants}

This study comprised of adolescents from grade 8 to 11 from four public secondary schools in Cape Town, South Africa ( $n=435$; boy: $46 \%$, girl: $54 \%$ ). The participants resided in communities where violent crimes are highly prevalent. The majority of the sample identified themselves as people of mixed heritage ( $74.9 \%), 21.8 \%$ were black, $1.9 \%$ were white, and $1.4 \%$ were Asian. Their ages ranged from 13 to 22 years $(M=16$ years, $S D=1.31)$.

\section{Procedure}

The data were collected by the primary author of the paper. The researcher first gained approval from the principals to collect data in their schools. The researcher decided with the principals to exclude Grade 12 adolescents in the study because they had to prepare for the final examinations. Convenient sampling was used to recruit participants available at the time of data collection, such as during their free/study period, to ensure the usual teaching activities were not disturbed. On the day before data collection, the researcher introduced herself, described the study background, study aims, and the importance of the study in front of the classes. The adolescents at the age of 18 years old or over who wanted to participate were provided consent forms, and the ones who were under 18 years old were given parental/caregiver consent forms and assent forms. The participants who brought back signed consent and/or assent forms were eligible to complete the questionnaire in the classroom. The completion time of the questionnaire was approximately 30 minutes and was under the supervision and monitoring of the researcher where participants had to independently complete the survey and could ask questions about the survey if they had any.

\section{Measures}

\section{Violence victimisation}

Three types of violence exposure were measured, namely indirect victimisation, crimerelated victimisation, and peer victimisation. The scales were adapted from the Juvenile Victimisation Questionnaire (JVQ, Finkelhor et al., 2005). All items were measured on a Likert scale, ranging from lower to higher intensity of exposure: Never (1) to Almost Always (5). Eight items were used to measure indirect victimisation, such as witnessing domestic violence, witnessing someone being assaulted with or without a weapon, or being present in an environment where there are street riots. Seven items were used to measure crimerelated victimisation, such as being a direct victim of property crime and physical assault with/without a weapon. Five items were used to measure peer victimisation, such as being physically assaulted by peers or siblings, and being a victim of bullying. The items of the three victimisation scales were averaged, with higher scores indicating more exposure to indirect victimisation $(\alpha=.68)$, crime-related victimisation $(\alpha=.79)$, and peer victimisation $(\alpha=.65)$ respectively.

\section{Violence perpetration}

Two types of violence perpetration were measured, namely, peer violence perpetration and delinquent behaviours. All items were measured on a Likert scale, ranging from lower to higher intensity of exposure: Never (1) to Almost Always (5). Peer violence perpetration scale was adapted from the peer victimisation scale of JVQ (Finkelhor et al., 2005), by changing the wording of victimisation to perpetration to measure behaviours such as physically assaulting peers or siblings, and being a perpetrator of bullying (5 items). Delinquent behaviours scale was adapted from the Survey of Children's Exposure to Violence (Richters \& Saltzman, 1990), by changing the wording of "victimisation" to "perpetration" to measure behaviours such as beating someone, mugging someone, and carrying a weapon (11 items). 
The items of the two perpetration scales were averaged, with higher scores indicating more peer violence perpetration $(\alpha=.60)$ and delinquent behaviours $(\alpha=.85)$.

\section{Protective factors}

Some of the protective factors in this study were measured by the Adolescent Resilience Questionnaire (Gartland et al., 2011) which provides a comprehensive measure of internal and external factors across different ecological contexts that may enhance positive adjustment and functioning in adolescents. The original scale comprises 12 scales that measure five domains of protective factors: self (self-confidence, emotional insight, negative cognition, social skills, and empathy); family (connectedness and availability); peers (connectedness and availability); school (supportive environment and connectedness); and community (connectedness).

For the purpose of this study, we included a total of eight scales, in which four of them measured internal protective factors, namely self-confidence, emotional insight, social skills, and empathy ( 8 items for each scale). Examples of these items were: feeling confident that one can handle difficulties (confidence), thinking things through before making decisions (emotional insight), expressing opinions when in a group (social skills), and being patient with others (empathy). Moreover, we included another four scales that measured external protective factors, namely family/peer/school/community connectedness $(8,7,8,6$ items, respectively). These scales entailed being in a positive environment and surrounded by supportive and reliable others. We did not include all the scales from family, peer, and school domains in the original questionnaire as these scales were moderately to highly correlated within each domain and the inclusion of only the connectedness scales may provide an adequate overview of social support from the major developmental contexts (Gartland et al., 2011). All items were measured on a Likert scale, ranging from lower to higher intensity of the stated feelings/thoughts: Almost Never (1) to Almost Always (5). The scales were averaged with higher scores indicating higher levels of confidence about self and future ( $\alpha=.82$ ), emotional insight ( $\alpha=.75)$, social skills $(\alpha=.66)$, empathy $(\alpha=.66)$, and family/ $\mathrm{peer} / \mathrm{school} / \mathrm{community}$ connectedness $(\alpha=.89 ; \alpha=.83 ; \alpha=.69 ; \alpha=.86$; respectively).

The 5-item anger management subscale of the Children's Emotion Management Scales (CEMS; Zeman et al., 2002) was used to measure participants' cognitive responses when they feel angry, for example, calmly deal with and think through ways to cope with anger. All items were measured on a Likert scale, ranging from lower to higher frequency of an anger management response: Almost Never (1) to Almost Always (5). Scores were averaged and higher scores indicate higher levels of anger management $(\alpha=.84)$.
The scale that measured participants' instrumental coping of negative emotions was adapted from the Brief Cope (Carver, 1997) to understand the behavioural coping responses when they feel angry or sad. For example, the use of religious practice and activities for selfdistraction. We further included an item on writing down feelings as a response to negative emotions - this item was informed by a prior study we conducted with another sample of South African adolescents on their coping processes following violence exposure, in which many of them find writing down feelings and journaling helpful to reduce sadness and anger (Sui, et al., submitted). All five items were measured on a Likert scale: Almost Never (1) to Almost Always (5). Scores were averaged and higher scores indicate higher levels of instrumental coping of negative emotions $(\alpha=.70)$.

The perceived effectiveness of Life Orientation was measured by one item, "Do you think Life Orientation in the school provides you with the skills you need to not engage in violent behaviours?" The item was measured on a Likert scale: No (1); No, it does not help for reducing violence but it helps for other things (2); Yes, but only a little bit (3); Yes (4). The scale was averaged with higher scores indicating higher levels of perceived usefulness of Life Orientation.

\section{Data Analysis}

The data were analysed using SPSS (Version 23). First, intercorrelations of sociodemographic variables, internal and external protective factors, and different types of violence victimisation and perpetration were examined, using point-biserial correlation tests at $\alpha=.01$ (2-tailed).

Next, multiple hierarchical regression analyses were conducted to examine the associations of protective factors with the five different types of violence, namely, witness violence, crimerelated victimisation, peer victimisation, peer perpetration, and delinquent behaviours. In Step 1 of the regression model, we entered sociodemographic variables of age, gender, race (reference group African), absence of father/mother in the household, and absence of both parents in the household. In Step 2, all the protective factors were entered. These include anger management, instrumental coping of negative emotions, self-confidence, emotional insight, social skills, empathy, family/peer/school/community connectedness, and perceived usefulness of Life Orientation. Since the regression analyses were conducted separately for the outcome variables, we applied Bonferroni correction by using a lower criterion for significance $(\alpha=.01)$ to minimise the potential for Type I error (Field, 2009). In addition, multicollinearity of the independent variables was diagnosed. The variance inflation factors (VIF) values were all under 2.33 for all variables and thus within the acceptable range (Hair, Black, Babin, Anderson, \& Tatham, 2006). 
Both results from the point-biserial correlations and multiple hierarchical regression analyses were interpreted to provide a comprehensive understanding of the determinants that are feasible to be targeted in intervention development. The results of the intercorrelations are valuable as they account for the potential overlap in the operationalisations of the psychological constructs which often covary (Peters \& Crutzen, 2018).

\section{Results}

\section{Prevalence of Victimisation and Perpetration in Adolescents}

In the 12 months preceding the survey, the frequency of exposure to each type of violence victimisation and violence perpetration was low (i.e., below the scale midpoint). However, the total number of exposure to different incidents of violence victimisation was high. All adolescents reported to have experienced at least one incident of victimisation $(100 \% ; n=$ $374)$ and the highest number of victimisation in the sample was 20 different incidents ( $M=$ 11 incidents). Furthermore, the majority (90\%) of the adolescents perpetrated at least one incident of violence in the 12 months preceding the survey. The actual range of the number of perpetration in the current sample was between 0 and 16 incidents ( $M=4$ incidents). Only $10 \%$ of the adolescents $(n=41)$ did not perpetrate any types of violence.

\section{Intercorrelations between Protective Factors and Different Types of}

\section{Violence}

Table 1 shows the point-biserial correlation tests of all variables, along with the results of the hierarchical multiple regression analyses.

The strength of the intercorrelations were catgeorised into weak $(r=.10-.23)$, moderate $(r=.24-.36)$, and strong $(r>.37)$ (Cohen, Cohen, West, \& Aiken, 2003). Age was positively and weakly associated with indirect victimisation. Being a boy was weakly associated with delinquent behaviours. Anger management was negatively and weakly associated with peer victimisation and delinquent behaviours, and moderately associated with peer violence perpetration. Instrumental coping of negative emotions was negatively and moderately associated with peer violence perpetration and weakly associated with delinquent behaviours. Self-confidence was negatively and weakly associated with peer victimisation. Emotional insight was negatively and weakly associated with peer victimisation and delinquent behaviours. Having social skills was negatively and weakly associated with peer victimisation. Empathy was negatively and moderately associated with peer violence perpetration, and weakly associated with delinquent behaviours.
Moreover, family connectedness was negatively and weakly associated with indirect victimisation and crime-related victimisation, and moderately associated with peer victimisation. Peer connectedness was negatively and weakly associated with crime-related victimisation and peer victimisation. School connectedness was negatively and weakly associated with peer victimisation, peer violence perpetration, and delinquent behaviours. Community connectedness was negatively associated with indirect victimisation, crimerelated victimisation, and peer victimisation. The perception of the usefulness of Life Orientation in school was negatively and weakly associated with delinquent behaviours.

\section{Multiple Hierarchical Regression}

The results of the hierarchical multiple regression analyses showed that sociodemographic variables accounted for $9 \%$ of the variance in indirect victimisation, $4 \%$ in crime-related victimisation, $3 \%$ in peer victimisation, $4 \%$ in peer violence perpetration, $7 \%$ in delinquent behaviours. The internal and external developmental assets accounted for an additional $11 \%$, $11 \%, 14 \%, 14 \%$, and $13 \%$ of variance in the five types of violence, respectively $(p \mathrm{~s}<.01)$.

In the final model, age $(\mathrm{B}=.11, p<.001)$, absence of both parents $(\mathrm{B}=.36, p<.01)$, and higher self-confidence $(\mathrm{B}=.21, p<.01)$ were associated with higher levels of indirect victimisation. Being a boy was associated with delinquent behaviours $(\mathrm{B}=-.20, p<.001)$. Lower levels of anger management was associated with higher indirect victimisation $(\mathrm{B}=-.13, p<.01)$ and delinquent behaviours $(\mathrm{B}=-.09, p<.01)$. Lower family connectedness was associated with higher indirect victimisation $(\mathrm{B}=-.14, p<.001)$, crime-related victimisation, and peer victimisation (both $\mathrm{Bs}=-.15, p \mathrm{~s}<.01$ ). Lower social skills was associated with higher peer victimisation $(\mathrm{B}=-.18, p<.01)$. Lower empathy was associated with higher peer violence perpetration $(\mathrm{B}=-.20, p<.001)$. Lower perceived the usefulness of Life Orientation lesson predicted higher delinquent behaviours $(\mathrm{B}=-.09, p<.01)$.

\section{Discussion}

This study examined the associations of internal and external protective factors with three types of violence victimisation and two types of violence perpetration in South African adolescents. The results suggested heterogeneity in the associations of different protective factors with each type of violence.

The results on the sociodemographic variables showed that older adolescents were at higher risk for indirect victimisation. Boys were at higher risk for delinquent behaviours. Indeed, as the age of the adolescents increases, the experiences of violence exposure may be compounded (Finkelhor et al., 2007). In addition, adolescents are typically impulsive 
and prone to thrill-seeking behaviours (Sui et al., 2020). Some degree of aggression is relatively common during this developmental period especially for boys (Logan-Greene, Nurius, Herting, Walsh, \& Thompson, 2010). Boys in particular are at higher risk than girls for externalising developmental problems such as aggression, aggressive fantasies, conduct problems, and higher levels of delinquency (Mrug, Loosier, \& Windle, 2008; Reingle \& Maldonado-Molina, 2012; Sharma \& Marimuthu, 2014). Moreover, adolescent boys in South Africa are more likely to spend time outside home than their female counterparts and more prone to the exposure to negative influences such as gangsterism and availability of alcohol and drugs, which may encourage the use of violence and delinquent behaviours (Sui et al., 2020).

In our study, adolescents' self-confidence was associated with lower levels of peer victimisation. Similarly, a longitudinal study showed that having positive domain-specific self-concepts such as higher perceived academic abilities and confidence in physical appearance in oneself, was associated with decreased levels of peer victimisation from childhood/early adolescents ages 10 to 12 to adolescents at age 15 (Blakely-McClure \& Ostrov, 2016). It may be the case that individuals with high self-confidence behave in a way that do not come across as "weak" and thus do not elicit bullying responses from peers. During adolescent years, the concept of self is important for identify formation, and can be dynamic and complex (Harter, 2006). Self-confidence in adolescents plays a vital role to promote positive adaption and psychosocial functioning (Gartland et al., 2011; Scales et al., 2000) and has been associated with higher life satisfaction (Ye, Yu, \& Li, 2012). To our surprise, we found that having self-confidence was associated with higher levels of indirect victimisation. It is possible that adolescents who have higher confidence are more likely to avail themselves in social situations and thus may be at higher risk for witnessing and hearing about violence. The current study prevents from drawing firm conclusions about this association and future research may provide additional insight into the underlying mechanism.

We found that having social skills was also protective for peer-victimisation. Victims of peer violence are often socially withdrawn and submissive as they generally avoid conflict with peers to limit opportunities for rejection (Schwartz, Proctor, \& Chien, 2001). Yet these characteristics contribute to the lack of social skills and put them at higher risk for victimisaiton (Fox \& Boulton, 2005). Since peer victimisation occurs in the context of social relationships, social skills could be an important protective factor. Social skills characterised by cooperation, assertiveness, and self-control may help adolescents become adept at reading social norms, facial expressions, mood, and intention of others (Holmes et al., 2015), which may help establish and maintain effective peer relations. Social skills may also be helpful in conflict provoking situations, as these skills enable an individual to de-escalate the conflict through various means, such as negotiation, humor, withdrawal, or self-assertion (Lepore \& Kliewer, 2019).

Furthermore, we found that having emotional insight was associated with less peer victimisation and delinquent behaviours. Emotional insight reflects an adolescent's introspective ability to understand and analyse the adverse situation, which gives more time to respond in ways that promote adaptive functioning, rather than quickly produces action tendencies (Gartland et al., 2011). Indeed, a study found that adolescents who are aware and attentive to their emotion of anger reported less histories of aggression than those who lacked such insight, suggesting that emotional insight helps adolescents control their violent behaviour when they are emotionally aroused, rather than controlling the negative emotions themselves (Roberton, Daffern, \& Bucks, 2015). In addition, emotions are driven by intraand interpersonal dynamics and are influenced by one's perception of a social situaiton, and can in turn influence the relations with others (Halperin \& Pliskin, 2015). Thus, being able to adaptively appraise negative emotions may help adolesents understand and respond to the emotion-eliciting situations appropriately, and give them the opportunity to establish functioning social relationships with peers which lower the risk for victimisation.

Another component of emotional competency is the ability to control negative emotions. We found that the cognitive ability to manage anger was associated with lower levels of indirect victimisation and peer victimisation. In addition, both cognitive anger management and instrumental coping of negative emotions of sadness and anger, were associated with peer violence perpetration and delinquent behaviours. This suggests that both cognitive- and instrumental coping of negative emotions may combat violent behaviours in adolescents. Perpetration of violence may be a way of expressing anger and achieve anger-driven goals (i.e., to inflict harm in the moment or to seek justice or plot revenge) (Sullivan et al., 2010). Thus, one possible mechanism of the association between inability to manage anger and violence perpetration may entail the intention to establish social hierarchies and maintain boundaries in interpersonal contexts (Sullivan et al., 2010). Conversely, individuals with effective emotion management may be better at exercising behavioural control and function better in social relationships, such as having effective communication and engaging with others in a prosocial manner (Kaynak, Lepore, Kliewer, \& Jaggi, 2015; Vanderbilt-Adriance \& Shaw, 2008). It is therefore possible that the ability to manage negative emotions lower the risk for adolescents to encounter and use violence in interpersonal contexts as they may be more capable to regulate and control their behaviours and resolve conflicts in a prosocial manner. 

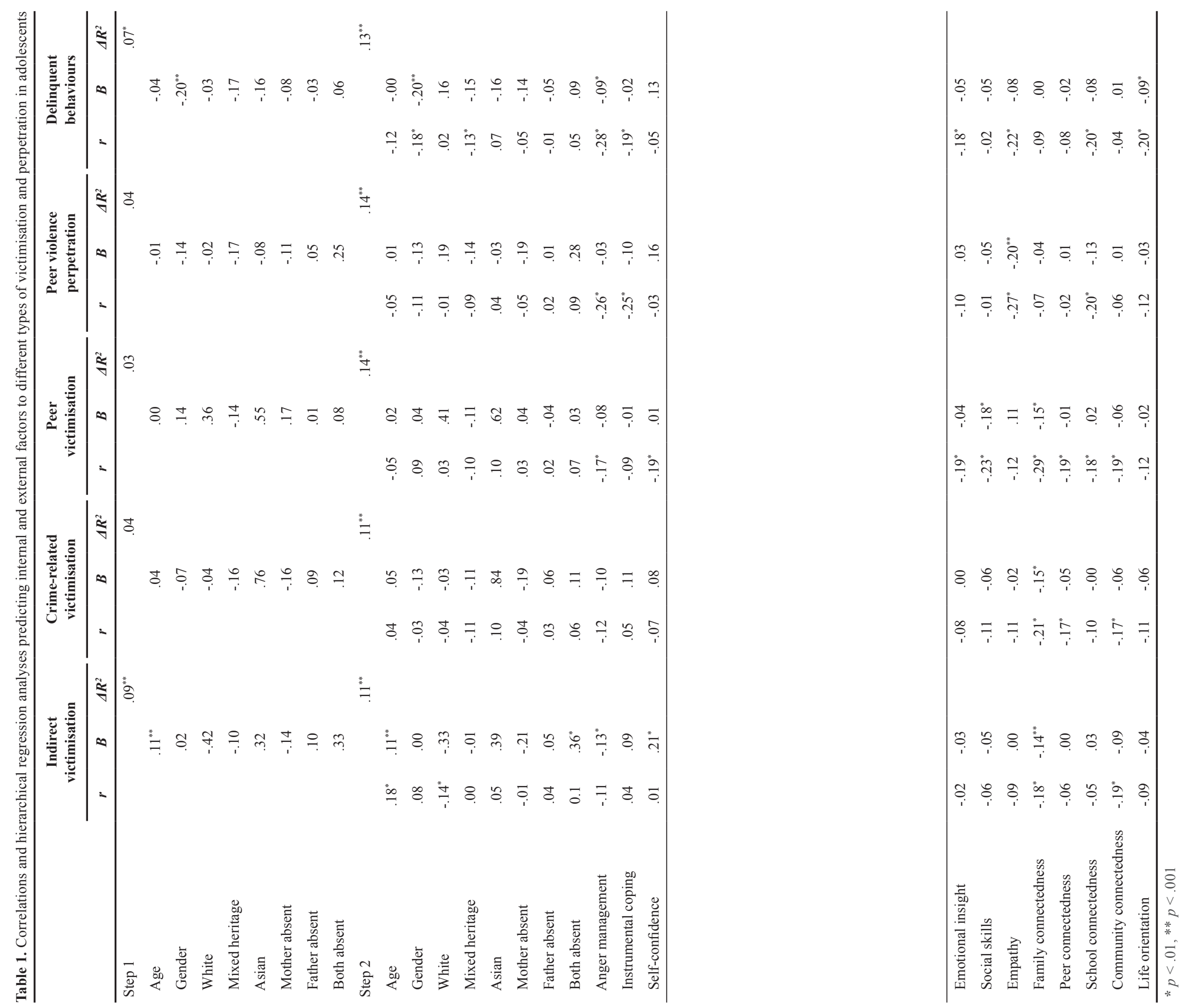
In line with other studies (Gini, Albiero, Benelli, \& Altoè, 2007; Zych \& Llorent, 2019), we found that empathy was associated with lower levels of peer violence perpetration and delinquent behaviours. Empathy has also been positively associated with prosocial behaviours that prevent violence such as standing up for and helping the victimised peers in school (Gini et al., 2007). More specifically, there are two components of empathy, namely cognitive empathy and affective empathy and both may mitigate aggressive behaviour and violence. The former entails the ability to appreciate and tolerate other people's perspective whereas the latter is the ability to experience other people's emotions and sharing their emotional states (Davis, 2006). It is possible that empathy allows adolescents to moderate their aggressive behaviour and anticipate the negative outcomes that may be produced by their own conduct and thus refrain from inflicting intentional harm on others.

Furthermore, studies have consistently established the protective role of social support from home, school, peers and, community in promoting positive adolescent adjustment and functioning (Kliewer et al., 2004; Scoglio et al., 2019). In our study, both parents' absence in the household was associated with higher levels of indirect victimisation. Our results also showed that family- and community connectedness were consistently associated with lower indirect victimisation, crime-related victimisation, and peer victimisation. Peer connectedness was associated with less crime-related victimisation, and both peer- and school connectedness were associated with less peer victimisation. These results suggest that the support across promixal and distal developmental contexts (i.e., home, peer, school, and community) may create safety, strengthen the supervision and monitoring of adolescents, and help them establish positive and reliable interpersonal relationships with others, which can prevent them from victimisation.

In particular, school connectedness was associated with less peer violence perpetration and delinquent behaviours. In South Africa, single parent households (Meintjes, Hall, Marera, \& Boulle, 2010) and parental substance use (Groenewald \& Bhana, 2018) are highly prevalent. As such, it is possible that adolescents may not be getting adequate support and supervision from home, and school is the only environment where they can receive structured education about anti-violence. When the needs of connectedness are met, adolescents may perceive that the adults in that particular setting are interested and concerned about them, and experience a heightened sense of self-worth, higher levels of self-regulation, and engage in developmentally healthy activities (Copeland-Linder, Lambert, \& Lalongo, 2010; Hogue, Liddle, Becker, \& Johnson-Leckrone, 2002). Therefore, a supportive school may help adolescents feel assured and effectively learn prosocial behaviours.
Lastly, the perceived usefulness of the skills that are taught in Life Orientation lesson to refrain from engaging in violence was associated with lower levels of delinquent behaviours in adolescents. As laid out in the National Curriculum Statement, Life Orientation in South African schools forms part of the life skills training to help young people become healthy and responsible individuals who are able to live productive lives in the new South African democracy (Department of Education, 2011). Currently, limited studies have evaluated the effectiveness of Life Orientation in South Africa. These studies have employed a qualitative approach and found that many learners perceived Life Orientation as unnecessary, boring and irrelevant (Jacobs, 2011), and many teachers felt that the effect of the Life Orientation teaching did not last (Prinsloo, 2007). However, our study provided a preliminary evidence that attending a structured education curriculum in school, such as Life Orientation that focuses on teaching skills to avoid the use of violence, may help reduce violent behaviours among adolescents.

\section{Implications for Interventions}

Strengths-based interventions have been a strong emphasis to enhance positive youth functioning and adjustment, and a few have been evaluated and shown effectiveness in enhancing personal skills and competencies to reduce the risk for victimisation and perpetration. For example, School-based Life Skills Training (Botvin, Griffin, \& Nichols, 2006) focused on building social and emotional competency in adolescents, specifically self-management skills (e.g., problem solving, stress management, anger management), social skills (e.g., effective communication, conflict resolution, assertiveness), and resistance skills (e.g., resist media influence of violence, resist peer pressure to the use of substance and aggression). The intervention showed a significant reduction in past year physical fighting and delinquent behaviour. It has previously also been found effective in preventing alcohol and illicit drug use in adolescents (Botvin et al., 2006), which are key factors that exacerbate the risk for violence exposure and violence perpetration (Duke, Smith, Oberleitner, Westphal, \& McKee, 2018).

While these interventions focus on building skills and competencies in individuals, multifaceted interventions that involve drawing support and resources from different ecological contexts are also likely to substantially promote positive youth functioning and behaviours. For example, Coping Power (Lochman et al., 2009) is a school-based intervention that incorporated both individual and family components. It aimed at enhancing problem solving, peer refusal, conflict management, coping and social skills in adolescents, as well as improving parents' ability to affective (e.g., warmth and bonding to children) and instrumental (e.g., consistent and appropriate monitoring, supervision, and reinforcement of behaviours) parenting practices. The intervention was delivered by trained school counsellors and was found effective in reducing adolescents' minor assault (hitting or threatening to 
hit others) two years post-intervention. Moreover, the Multidimensional Family Prevention programme (Hogue et al., 2002) entailed 15 to 20 sessions with both adolescents and parents to strengthen family functioning, and help them establish and engage effectively in the interactions within their social network (e.g., family, school, prosocial peer groups, and recreational and religious institutions). This programme has shown to have immediate positive impact on adolescents' self-confidence, school bonding, and a decreased level in their antisocial behaviours.

Since South African adolescents are at risk for violence exposure across multiple developmental contexts and that both internal and external protective factors are associated with victimisation and perpetration experiences, we think that a multi-setting and multifaceted approach may be effective for the development of prevention programmes. Based on our results, it may be helpful to enhance empathy and promote social awareness of recognising and appreciating individual and group differences, as well as to foster selfmanagement of negative emotions, to reduce the risk for violence perpetration among adolescents. The delivery of these skills and competencies can be integrated in the national education curriculum in South African schools, such as Life Orientation, with an opportunity to revise the content of the current curriculum. School connectedness may be strengthened by enhancing adolescents' feelings of commitment to school both socially and academically, to complement the learning of skills in school to reduce violent behaviours. Additionally, it may be helpful to involve family in the intervention and build emotion regulation skills in parents/caregiver to help minimise the risk for their children's violent behaviours. As shown in a study, higher levels of caregiver's anger regulation was protective for their children's externalising symptoms (Kliewer et al. 2004). This suggests that caregivers/parents with high emotional regulation skills may model emotional control and adaptive coping of stressors, which may help adolescents appraise their feelings, learn about strategies for anger management, and achieve competence in controlling anger and violent behaviours.

Similarly, multifaceted programmes can be adopted to prevent violence victimisation among adolescents. Based on our results, it is helpful to enhance adolescents' social skills, self-confidence, emotional insight, and anger management, and also incorporate training to forge durable interpersonal connections and engage effectively in the interactions within their social network. For example, an improved supervision, monitoring, and support from family and school may help adolescents develop competencies to stay away from risky situations where they may be victimised (Sui, Massar, Reddy, \& Ruiter, 2020). Interventions that prevent violence victimisation in South African adolescents may be helpful to involve family members, peers, teachers, and important stakeholders from the community to extend and maximise the support in adolescents' developmental contexts to keep them safe from violence exposure.

\section{Limitations and Future Directions for Research}

While this current study provided a refined overview of the associations of internal and external protective factors with different types of violence, several limitations shall be noted. Firstly, this study was cross-sectional in nature. Thus causality between protective factors and violence victimisation and perpetration may not be inferred, and possible bi-directional associations should be taken into consideration. Longitudinal studies may provide further insight into the mechanism of the associations.

In our study, we measured a range of distinct and separate internal and external protective factors. Although each factor has been operationalised and discussed separately, others studies have combined, for example, emotional insight, anger management, and social skills into a composite measure of social intelligence (Lepore \& Kliewer, 2019), and adult support from home and school contexts as a composite measure of social support (Choe, Zimmerman, \& Devnarain, 2012). Cautions may be applied when interpreting the results with others studies as the operationalisations may differ.

Moreover, this study relied on adolescent self-report measures. An inflated associations between variables may be possible, as adolescents can answer the questionnaire in a way that appears to be self-fulfilling and socially desirable. Future studies could explore different methods of assessment, such as teachers' and parents' observations.

In addition, studies have established an association between victimisation and perpetration as the two experiences may overlap in the same adolescents (Jeong et al., 2015; Reingle \& Maldonado-Molina, 2012). The subgroup of adolescents who are both a victim and a perpetrator of violence (victim-perpetrators) may be important to consider as it marks a substantial proportion of $24.6 \%$ in a nationally representative sample of South African inschool adolescents (Sui, Massar, Ruiter, \& Reddy, 2020). However in the current study, we were unable to distinguish the sample into victims only, perpetrators only, victimperpetrators, and non-involvement in both victimisation and perpetration, as all adolescents in the sample have experienced at least one type of victimisation. As such, the subgroups of perpetrators only and non-involvement do not exist in our sample which prevented us from providing clear cuts of violence typologies and their associated protective factors. Future studies may consider differentiating violence into more refined typologies where possible to help inform tailored interventions. 


\section{Chapter 4}

\section{Violence Exposure in South African Adolescents: Differential and Cumulative Effects on Psychological Functioning}




\section{Abstract}

This study examined the associations between different types of violence victimisation and psychological functioning in South African adolescents. Both differential and cumulative effects of violence were investigated. A multi-ethnic (black, white, people of mixed heritage, and people of Indian/Asian descent) sample of adolescents in secondary schools in the Western Cape Province ( $n=1574$; boy: $46.5 \%$, girls: $53.5 \% ; M$ age $=16$ years) completed a survey on their experiences of exposure to violence (across different contexts and poly-victimisation) and their levels of hopelessness, anxiety, depression, perceived stress, and suicidal ideation. The results showed that indirect and direct victimisation in the community, and indirect political victimisation were consistent predictors for adverse psychological functioning, whereas victimisation in home and school contexts did not emerge to be significant. Polyvictimisation had a consistent linear effect on psychological symptoms. Interventions in South Africa should focus on addressing the psychological effects of community and political victimisation on adolescents. Adopting a holistic treatment approach would be useful to gain a comprehensive understanding of adolescents' victimisation experiences and maximise the impact of support to enhance their psychological functioning.
Reducing violence exposure is a global public health priority, particularly with respect to promoting health and wellbeing in adolescents (World Health Organization [WHO], 2014). Violence is a significant risk factor for adverse psychological functioning in adolescents (WHO, 2014). South African adolescents are at increased risk for psychological maladjustment due to the country's alarmingly high rates of crime and violence. On a daily basis, an average of 51 cases of murder, 142 cases of sexual offence, and 452 cases of common assault are recorded (South African Police Service, 2016). Within a violence-prone environment, interpersonal violence encountered by South African adolescent boys and girls is eight and five times higher than the global average, respectively (Norman, Matzopoulos, Groenewald, \& Bradshaw, 2007). At the same time, mental health programmes for youth in South Africa are inadequately funded and sourced (Lund, Boyce, Flisher, Kafaar, \& Dawes, 2009; Petersen, Swartz, Bhana, \& Flisher, 2010), which adds to these adolescents being at higher risk for psychological vulnerabilities because they are unable to access adequate mental health support in instances of victimisation. Given the limited resource setting in South Africa, it is imperative to examine the psychological effects of violence on adolescents to inform future violence reduction and health interventions.

\section{Violence Exposure across Contexts}

Violence can be experienced indirectly (witnessing or hearing about violence that happened to others) or directly (personal experience of intentional acts initiated by another person to cause harm) and across different contexts in an adolescent's life, such as home (e.g., domestic violence; Hindin \& Gultiano, 2006), school (e.g., bullying; Perren, Dooley, Shaw, \& Cross, 2010), community (e.g., violent crime; McDonald, Deatrick, Kassam-Adams, \& Richmond, 2011), and a context of violence motivated by political reasons (e.g., IsraeliPalestinian conflict; Boxer et al., 2013). A study in the Western Cape Province of South Africa found that almost all adolescents (98.9\%) had witnessed community violence, $40.1 \%$ had been a direct victim of community violence, $76.9 \%$ had witnessed home violence, $58.6 \%$ had been a direct victim of home violence, and $75.8 \%$ had had either direct or indirect exposure to school violence (Kaminer, du Plessis, Hardy, \& Benjamin, 2013).

In addition, owing to the colonial history of South Africa, the younger generations may have heard stories of the brutality and discrimination that happened during Apartheid ${ }^{1}$, against their relatives, friends, or someone they know. The Apartheid regime in the past has contributed to the persistent disparities in people's socioeconomic status, health, and access to healthcare across ethnic groups in contemporary society, including mental health outcomes (Coovadia, Jewkes, Barron, Sanders, \& McIntyre, 2009). More specifically, the legacy of Apartheid is reflected in the inequitable mental health status of young South Africans - in a sample of adolescents in Cape Town, anxiety, depression, and posttraumatic stress disorder are more significantly associated with adolescents in 
historically disadvantaged ethnic groups (black and people of mixed heritage) than with white adolescents (Das-Munshi et al., 2016).

\section{The Effects of Violence on Psychological Functioning}

From a developmental perspective, adolescents may be particularly vulnerable for the psychological effects of violence, because mental health problems are likely to emerge during this stage in life due to the maturational changes that occur in the brain (Botticello, 2009; Paus, Keshavan, \& Giedd, 2008). Violence exposure has been found to disrupt adolescents' adaptive psychological functioning in many ways. For example, since violence is inherently stressful (Mrug, Loosier, \& Windle, 2008), it may interfere with and exacerbate exposure to daily stressors and disrupt a normative stress adaptation process (Heinze, Stoddard, Aiyer, Eisman, \& Zimmerman, 2017). Violence exposure may further dampen individuals' goals and aspirations and elicit feelings of hopelessness through perceptions that there are few pathways for recovery and progress in life (Bonanno, 2007). Moreover, depression and anxiety are common symptoms in adolescents exposed to violence (Bach \& Louw, 2010; Mrug et al., 2008; Mrug \& Windle, 2010), which may lead to suicidal ideation and suicide attempts (Bonanno, 2007; Pillai, Andrews, \& Patel, 2009). Globally, suicide is one of the leading causes of mortality among young people (McKinnon, Gariépy, Sentenac, \& Elgar, 2016), and violence exposure may increase the risk for suicidal ideation in adolescents (Bonanno, 2007, Saewyc \& Chen, 2013). In addition, when the psychological problems experienced in adolescence are not identified and treated in an early stage, they may persist and have a direct impact on adolescents' future functioning and mental health in adulthood (Botticello, 2009; Bruwer et al., 2014; Das-Munshi et al., 2016). Given the impact violence exposure has on adolescents' long-term psychological functioning, it is important to understand these effects of violence to inform mental health interventions.

Relatively less research has cut across multiple contexts to investigate the effects of violence (Dubow et al., 2010). Yet, violence may have differential effects on adolescent psychological functioning depending on the particular context in which the victimisation occurs. Mrug et al. $(2008,2010)$ found that violence that occurs in proximal contexts, such as home and school, produces more harmful psychological effects (anxiety and depression) than violence exposure in distal environments such as community. Home and school are the major developmental domains in an adolescent's life and the exposure in these contexts may undermine the quality of important relationships. The resulting absence of secure and supportive relationships may thus foster negative psychological symptoms (Mrug et al., 2008; Mrug \& Windle, 2010). Examining the differential effects of violence exposure across multiple contexts may enhance the understanding of the environment in which the adolescents are vulnerable to psychological maladjustment and provide refined and targeted recommendations for mental health care.

\section{Poly-Victimisation}

Traditionally, research on victimisation has focused on discrete and single types of violence (e.g., sexual violence) without investigating the other exposures that the same children or adolescents may encounter (Finkelhor, Ormrod, \& Turner, 2007; Finkelhor, Turner, Hamby, \& Ormrod, 2011). This can potentially fail to obtain the full victimisation profile as violence is rarely an event in isolation; instead, victimisation experiences are interconnected and may cluster and occur concurrently (Finkelhor et al., 2007; Finkelhor, Ormrod, Turner, \& Holt, 2009; Finkelhor et al., 2011; Kaminer et al., 2013; Leoschut \& Kafaar, 2017). Adolescents who are exposed to one form of violence are at increased risk to be exposed to violence in other forms, suggesting that victimisation experiences can accumulate (Finkelhor et al., 2007). The exposure to multiple and different types of violence is known as polyvictimisation (e.g., Finkelhor et al., 2007) and is a common experience for South African adolescents (Kaminer et al., 2013). In a nationally representative sample of 15-17 year old South Africans, $64 \%$ had experienced two or more different types of victimisations ever in their lives, and $35.4 \%$ had experienced five or more types of different victimisations (Leoschut \& Kafaar, 2017). More information on the effects of poly-victimisation on adolescents is needed in South Africa as most research is conducted in the United States and European countries (Pereda, Guilera, \& Abad, 2014).

Poly-victims are particularly at high risk for maladjustment in psychological functioning and significant associations have been found between poly-victimisation and anxiety, depression, and perceived stress in children and adolescents (Gustafsson, Nilsson, \& Svedin, 2009; Heinze et al., 2017; Latsch, Nett, \& Humbelin, 2017; Soler, Paretilla, Kirchner, \& Forns, 2012). More specifically, an increasing number of victimisation is associated with a parallel increase in psychological maladjustment (Ellonen \& Salmi, 2011; Finkelhor et al, 2007; Mrug et al., 2008).

However, the relationship between poly-victimisation and psychological functioning is not always linear. Mrug et al. (2008) observed a quadratic function, i.e., an inverted U-shaped curvilinear effect between poly-victimisation and anxiety and depression in adolescents, represented by an initial linear increase in symptoms at low to moderate levels of violence exposure (0-3 victimisations), and then levelling off and reaching a plateau at moderate levels (4-9 victimisations), followed by a slight decrease at high levels of exposure (1012 victimisations). Similarly, Morales and Guerra (2006) found that cumulative stress experienced by children in family, school, and community showed an initial linear increase in depression until the three stressor level (exposure to three types of stress across contexts) and then a levelling off in depression scores between three and six stressors. Although the quadratic relationship between poly-victimisation and psychological functioning has not yet been explored in South Africa, a study found that exposure to community violence 
was generally higher in South African children compared to American children. The South African sample experienced less psychological distress than their American counterparts, which could be due to South African children viewing community violence as normative and perceive it as less intense (Shields, Nadasen, \& Pierce, 2013). Thus, it may be possible that South African adolescents in the current sample experience less negative psychological symptoms when the number of violence exposure reaches a certain threshold, as they become psychologically desensitised to poly-victimisation.

\section{This Study}

While there have been many studies focusing on the psychological effects of a single type of violence (e.g., community violence, Chen, 2010; McDonald et al., 2011; bullying, Perren et al., 2010), researchers are calling for the need to measure violence exposure and its adverse effects in a more comprehensive fashion, since violence in its different forms and across different contexts may be differentially associated with psychological functioning (Finkelhor, Turner, Ormrod, Hamby, \& Kracke, 2009; Kadra, Dean, Hotopf, \& Hatch, 2014).

Given the high prevalence of violence in contemporary South Africa, South African adolescents can be directly or indirectly exposed to violence across major domains in their lives, namely, home, school, community, as well as experiencing politically-driven violence. Moreover, they can experience poly-victimisation across these contexts. Examining the differential and cumulative effects of violence across contexts will enhance the understanding of the circumstances in which the adolescents are at risk for the key psychological correlates informed by the literature, such as hopelessness, anxiety, depression, perceived stress, and suicidal ideation. This may provide insight into the prevention of future adverse functioning in adolescents and inform policies and mental health interventions in South Africa.

In this study, we examine the associations between different types of violence exposure and the psychological functioning in South African adolescents. We investigate the effects of violence exposure across multiple contexts in South African adolescents' lives (home-, school-, community- and political victimisation) on indices of psychological functioning, namely, hopelessness, anxiety, depression, perceived stress, and suicidal ideation. We also explore the cumulative effects of poly-victimisation on psychological functioning, by modelling both linear and quadratic relationships between these variables. Based on the literature, we hypothesize the following: Violence exposure in proximal contexts (home and school) and poly-victimisation are associated with negative psychological functioning in adolescents. We expect an inverted U-shaped curvilinear effect of poly-victimisation on psychological functioning, as indicated by an initial rise in the levels of psychological symptoms then a levelling off and/or decrease in the levels of symptoms as the number of violence exposure increases.

\section{Method}

\section{Participants}

The final sample consisted of 1574 adolescents from Grade 8 to 11 (this covers the age range 13 - 20 in the South African context) (boy: 46.5\%; girl: 53.5\%). Their age ranged between 12 and 22 years $(M=16$ years for both boys and girls, $S D=1.59$ and 1.55 , respectively). Forty percent were black, 51\% were people of mixed heritage, $1.6 \%$ were people of Indian/ Asian descent, and $6.9 \%$ were white. Twenty-two percent lived in rural area, 27.6\% lived in urban area, and $21.1 \%$ lived in peri-urban. The majority lived in formal dwellings ${ }^{2}(81.6 \%)$. Most participants' father/male guardian and mother/female guardian were employed (70.3\% and $63 \%$, respectively).

\section{Procedure}

The study was approved by the Ethics Committee of the Medical Research Council in South Africa. Data were collected by the fourth author in 2011 and 2012. A two-stage cluster sampling procedure was used (see also Kann et al., 2014). In stage one, a list of public schools in the Western Cape Province was obtained from the South African National Department of Education. Next, twenty-three public schools were selected with a probability proportional to student size, i.e., larger schools had a greater probability to be selected (schools with an enrolment of $>25$ learners per grade were considered as large, those with $<25$ learners per grade were small). In stage two, classes from grades 8 to 11 were selected using systematic equal probability sampling from each selected school. All learners in the selected classes were eligible to participate. Each participant was given an information packet to take home that included the background of the study, assent/consent forms, and referral information on violence treatment programmes and substance abuse treatment. For those who were under the age of 18 years, parental consent was required along with the assent forms. The participants were told to bring their signed assent/consent forms to school the next day and the ones who agreed to participate were invited to complete a questionnaire in their classrooms.

\section{Measures}

Sociodemographics. We gathered information on participants' gender, age, ethnic group (black, white, people of mixed heritage, people of Indian/Asian descent), dwelling type (formal vs. informal dwelling), area of living (urban, rural, peri-urban), and father/male guardian' and mother/female guardian's employment status.

Indirect home victimisation. Indirect exposure to home violence was measured using two items adapted from the Survey of Children's Exposure to Violence (Richters \& Saltzman, 1990): "How many times in your whole life have you seen people chased by someone who 
wanted to hurt them in your home?" and "...have you seen people beaten up by someone in your home?" The original items do not include context related wording because the instrument was designed to measure exposure to community violence. To measure indirect home victimisation, we added "in your home" to these items. The responses were 1 (never), 2 (once or twice), 3 (a few times), and 4 (many times), and were averaged to form an index of indirect home victimisation. Higher scores indicated higher frequency of indirect home victimisation $(r=.40)$.

Indirect school victimisation. Similar to indirect home victimisation, indirect school victimisation was measured using the same two items as home victimisation, except that the wordings were changed to "in your school". Higher scores indicated higher frequency of indirect school victimisation $(r=.47)$.

Indirect community victimisation. Indirect exposure to community violence was measured using fifteen items adapted from the Survey of Children's Exposure to Violence (Richters \& Saltzman, 1990) to suit the South African context of violence and crime. These items assessed the frequency of an adolescent witnessing or hearing about different types of violence in the community in his or her lifetime. Fifteen types of violence exposure in the community included witnessing or hearing about physical violence, threats, deaths, and interpersonal crime, e.g., "How many times in your whole life have you seen people slap, hit, or punch someone?" and “...have you seen or heard about people killed by someone else?" Response options were 1 (never), 2 (once or twice), 3 (a few times), and 4 (many times). Items were averaged and higher scores indicated higher frequency of indirect community victimisation $(\alpha=.90)$

Direct community victimisation. Similar to indirect community victimisation, the items measuring direct community victimisation were adapted from the Survey of Children's Exposure to Violence (Richters \& Saltzman, 1990). A total of fifteen items were used to measure fifteen types of violence related to an adolescent's direct experience of physical violence, threats, and interpersonal crime in the community in his or her lifetime, e.g., "How many times in your whole life have you, yourself, actually been attacked or stabbed by someone with a knife, panga and/or kierrie?" and “...has someone threatened or tried to kill you?" Response options were 1 (never), 2 (once or twice), 3 (a few times), and 4 (many times). The scores were averaged and higher scores indicated higher frequency of direct community victimisation $(\alpha=.84)$.

Indirect political victimisation. The indirect exposure to political violence scale was adapted from a previous study (Whitbeck, Hoyt, McMorris, Chen, \& Stubben, 2001) to measure the experience of Apartheid. Adolescents were asked whether they heard of or know any of South Africa's colonial and historical events of Apartheid that happened to someone in their lives, such as a family, a friend or an acquaintance. The scale has twentyeight items related to the political violence including forced removal and denied access of entry, participation in political uprising activities, experience of being imprisoned, and incidents of racial discrimination, e.g., "Have any of your family/friends or anyone you know ever been removed from home and forced to move somewhere else because of race?" and "...ever experienced police brutality (beaten, harassed) because of race?" The response options were 1 (yes), 2 (no), and 3 (I don't know). The "yes" responses were summed to obtain an index of indirect exposure to political violence with higher scores representing more exposure in an adolescent's life (possible range $0-28 ; \alpha=.83$ ).

Poly-victimisation. Poly-victimisation was computed to indicate the additive count of one's violence exposure by summing the number of violence each adolescent has indirectly or directly experienced, after first recoding the items of all victimisation variables into 1 (exposure) and 0 (no exposure). The indication of any frequency of a particular victimisation item was coded as 1 . Adolescents who reported "never" to the items measuring the different types of victimisation were coded as 0 (possible range $0-62$; actual range 0 to $61, M=41$, $S D=7.51)$.

Hopelessness. Feelings of hopelessness were measured using Beck's Hopelessness Scale (Beck, Weissman, Lester, \& Trexler, 1974). The scale consisted of five dichotomous items 1 (true) or 0 (false), e.g., "I might as well give up because I can't make things better for myself." and "I never get what I want, so it's dumb to want anything." The scale was summed and the higher score indicated higher level of hopelessness (possible range $0-6 ; \alpha$ $=.75$ ). The normality tests showed the responses were positively skewed and approximately half of the participants reported being not hopeless. Therefore, this variable was further dichotomized into " 0 " for the participants who scored 0 for the summed score, and "1" for the ones who scored 1 or higher for the summed score (hopeless: $38 \%$, not hopeless: $42 \%$ )

Anxiety. Anxiety symptoms were measured using the Revised Children's Manifest Anxiety Scale (Reynolds \& Richmond, 1985). The twenty-eight items measured physiological symptoms, worry/oversensitivity, and concentration problems an adolescent had in difficult situations, e.g., "I worry about what other people think of me." and "I get nervous when things do not go the right way." Adolescents were asked to respond with 1 (yes) or 0 (no) The "yes" responses were summed to compute the final score with higher scores indicating more feelings of anxiety (possible range $0-28 ; \alpha=.87$ ).

Depression. Depression symptoms were examined using a 10-item scale of the Center for Epidemiological Studies for Depression (Radloff, 1977). Respondents were asked to 
indicate how often they experienced certain thoughts/emotions during the past week on a five point scale - 1 (never), 2 (hardly ever), 3 (sometimes), 4 (quite often), and 5 (always), e.g., "I was bothered by things that usually don't bother me." and "I felt fearful." Positively phrased items (e.g., "I felt hopeful about the future.") were reverse coded before computing an average score across the items of the scale, so that higher scores mean more symptoms of depression $(\alpha=.80)$.

Perceived stress. The degree to which the adolescents perceived their lives as stressful was measured using the 10-item Perceived Stress Scale (Cohen, Kamarck, \& Mermelstein, 1983). Adolescents were asked to indicate how often during the past month they found their lives unpredictable, uncontrollable, and overloaded on a scale with responses of 1 (never), 2 (hardly ever), 3 (sometimes), 4 (quite often), and 5 (always), e.g., "How often have you felt that things were going your way?" and "How often have you found that you could not cope with all the things that you had to do?" Positively worded items were recoded before computing an average score across the items of the scale, so that higher scores indicate more perceived stress $(\alpha=.84)$.

Suicidal ideation. Suicidal ideation was measured by two items from the $3^{\text {rd }}$ National Youth Risk Behaviour Survey in South Africa (Reddy et al., 2013). Adolescents were asked about their contemplation of taking some action to end their own lives in the past six months: "Did you ever seriously consider attempting suicide?" and "Did you make a plan about how you would attempt suicide?" The responses were binary, with 1 (yes) and 0 (no). The two items were summed to form an index of suicidal ideation $(r=.63)$. The normality test showed the responses were positively skewed, with more than $60 \%$ of adolescents did not have any suicidal ideation. As such, the variable was dichotomized into " 0 " for the participants who scored 0 for the summed score, and " 1 " for the ones who scored 1 or higher for the summed score (have ideation: $22 \%$, no ideation: $64 \%$ ).

\section{Data Analysis}

The data were analysed using SPSS (version 23). For the analyses, we examined the levels of exposure to different types of violence and the intercorrelations between violence and the psychological correlates to understand the magnitude of violence exposure and the significance of the correlations between the variables. Independent samples $t$-tests were conducted to investigate the gender differences in violence exposure and psychological functioning. We also examined the differential effects of violence across contexts on psychological functioning, by conducting regression analysis separately for each outcome variable. Multiple hierarchical regression was conducted for the outcome variables that are continuous (anxiety, depression, perceived stress), whereas binary logistic regression was conducted for outcome variables that are dichotomous (hopelessness, suicidal ideation). In step 1 of the regression model, we entered only the sociodemographics that showed consistent associations - i.e., more than one significant association with the predictor and outcome variables, namely, gender, people of mixed heritage, white, dwelling type, peri-urban, and father employment. In step 2 of the regression model, victimisation variables were entered. To examine the effects of poly-victimisation and its quadratic function on psychological correlates, step 1 of the regression model remained the same and poly-victimisation and its quadratic function were entered in step 2 . The analyses were done separately by multiple hierarchical regression or logistic regression for each outcome variable. The quadratic function was calculated by first centering the poly-victimisation variable to reduce multicollinearity then calculating the square of the centered variable.

In addition, since the regression analyses were conducted separately for the five outcome variables, we applied Bonferroni correction by using a lower criterion for significance ( $\alpha=$ 0.01) to minimise the potential for Type I error (Field, 2009).

\section{Results}

Table 1 shows the mean scores of the frequencies of exposure to violence in different contexts and poly-victimisation. Within each context, the frequency of violence exposure among adolescents was low (i.e., below the scale mid-point), but poly-victimisation was high (average 41 out of maximum 62). There were significant gender differences in violence exposure: girls reported more indirect home victimisation than boys, $t(1124)=-1.97, p<$ .05 ; boys reported more indirect school victimisation, $t(1133)=2.51, p<.05$, and direct community victimisation, $t(1101)=2.18, p<.05$, than girls.

Table 1. Means (and standard deviations) of the frequencies of exposure to different types of victimisation in adolescents

\begin{tabular}{lccccccc}
\hline & \multicolumn{3}{c}{ Total } & \multicolumn{3}{c}{ Boys } & \multicolumn{3}{c}{ Girls } \\
\hline Type of Exposure & $\mathrm{n}$ & $M(S D)$ & $\mathrm{n}$ & $M(S D)$ & $\mathrm{n}$ & $M(S D)$ & $\alpha / r$ \\
Indirect home victimisation & 1,283 & $1.62(0.74)$ & 522 & $1.58(0.72)$ & 604 & $1.66(0.76)$ & .40 \\
Indirect school victimisation & 1,296 & $2.37(0.90)$ & 520 & $2.45(0.92)$ & 615 & $2.32(0.87)$ & .47 \\
$\begin{array}{l}\text { Indirect community } \\
\text { victimisation }\end{array}$ & 1,314 & $2.36(0.67)$ & 532 & $2.36(0.69)$ & 619 & $2.33(0.64)$ & .90 \\
$\begin{array}{l}\text { Direct community } \\
\text { victimisation }\end{array}$ & 1,315 & $1.43(0.40)$ & 533 & $1.45(0.40)$ & 618 & $1.40(0.38)$ & .84 \\
Indirect political victimisation & 1,310 & $4.54(5.49)$ & 531 & $4.56(5.67)$ & 617 & $4.31(5.23)$ & .83 \\
Poly-victimisation & 1,312 & $41.22(7.51)$ & 532 & $41.38(8.17)$ & 618 & $41.10(6.85)$ & - \\
\hline
\end{tabular}


Weak to strong positive correlations were found among the five types of victimisation ( $r$ 's ranging from .07 to $.53, p$ 's $<.01$ ). The intercorrelations between different types of victimisation and psychological symptoms showed a weak to moderate effect with $r$ 's ranging from .07 to .26 ( $p$ 's <.05), suggesting that a higher frequency of a type of victimisation exposure was associated with higher levels of adverse psychological functioning. Lack of associations were found for indirect school victimisation, indirect community victimisation, and poly-victimisation with hopelessness. Similarly, lack of associations were found for indirect community victimisation with suicidal ideation, as well as indirect political victimisation with depression and perceived stress ( $p$ 's >.05).

Lastly, gender was positively but weakly associated with all dependent variables except for hopelessness. Being a girl was associated with more psychological maladjustment than boys ( $r$ 's ranging from .11 to .21, $p<.01$ ). Inspection of the differences between girls and boys indicated that girls reported more anxiety $t(1118)=-7.28, p<.001$, depression $t(1126)=$ $-5.63, p<.001$, perceived stress $t(1071)=-4.04, p<.001$, and suicidal ideation, $\chi^{2}(1)=$ $15.91, p<.001$, than boys.

Table 2. Hierarchical regression analyses of sociodemographics and violence across different contexts on psychological functioning in adolescents

\begin{tabular}{|c|c|c|c|c|c|c|}
\hline & \multicolumn{2}{|l|}{ Anxiety } & \multicolumn{2}{|c|}{ Depression } & \multicolumn{2}{|c|}{ Perceived Stress } \\
\hline & $B$ & $\Delta \boldsymbol{R}^{2}$ & $B$ & $\Delta \boldsymbol{R}^{2}$ & $B$ & $\Delta \boldsymbol{R}^{2}$ \\
\hline Step 1 & & $.09 * * *$ & & $.06 * * *$ & & $.07 * * *$ \\
\hline Gender & $.23 * * *$ & & $.17 * * *$ & & $.11^{* * *}$ & \\
\hline Mixed heritage & $-.17^{* * *}$ & & $.11 * *$ & & $.17 * * *$ & \\
\hline White & $-.19 * * *$ & & .00 & & .06 & \\
\hline Dwelling & .02 & & .04 & & $.10^{* *}$ & \\
\hline Peri-urban & -.02 & & -.06 & & -.05 & \\
\hline Father employment & .02 & & .03 & & .04 & \\
\hline Step 2 & & $.07 * * *$ & & $.06 * * *$ & & $.05 * * *$ \\
\hline Gender & $.25 * * *$ & & $.18 * * *$ & & $.12 * * *$ & \\
\hline Mixed heritage & $-.10^{*}$ & & $.15 * * *$ & & $.18^{* * *}$ & \\
\hline White & $-.13^{* *}$ & & .06 & & $.10^{*}$ & \\
\hline Dwelling & .02 & & .02 & & $.07 *$ & \\
\hline Peri-urban & -.01 & & $-.07 *$ & & $-.07 *$ & \\
\hline Father employment & .03 & & .05 & & .05 & \\
\hline Indirect home victimisation & .03 & & .05 & & .03 & \\
\hline Indirect school victimisation & -.01 & & .01 & & .07 & \\
\hline Indirect community victimisation & -.01 & & .08 & & $.12^{* *}$ & \\
\hline Direct community victimisation & $.19 * * *$ & & $.16^{* * * *}$ & & .07 & \\
\hline Indirect political victimisation & $.16^{* * * *}$ & & -.03 & & -.06 & \\
\hline
\end{tabular}

$* p<.05 * * p<.01 * * * p<.001$

\section{Differential Effects of Victimisation on Psychological Functioning}

The results of the hierarchical multiple regression showed that sociodemographic variables accounted for $9 \%$ of variance in anxiety, $6 \%$ of variance in depression, and $7 \%$ of variance in perceived stress. The five types of victimisation accounted for an additional $7 \%, 6 \%$, and $5 \%$ of variance for anxiety, depression, and perceived stress, respectively. In the final model, gender was a consistent predictor for anxiety, depression, and perceived stress (B's $>.12, p$ 's $<.001)$. Unique predictors for anxiety were direct community victimisation $(\mathrm{B}=.19, p<.001)$ and indirect political victimisation $(\mathrm{B}=.19, p<.001)$. Direct community victimisation was a unique predictor for depression $(\mathrm{B}=.16, p<.001)$, and indirect community victimisation was a unique predictor for perceived stress $(\mathrm{B}=.12, p<.001)$.

Table 3. Binary regression analyses of sociodemographics and violence across different contexts on psychological functioning in adolescents

\begin{tabular}{|c|c|c|c|c|}
\hline & \multicolumn{2}{|c|}{ Hopelessness } & \multicolumn{2}{|c|}{ Suicidal Ideation } \\
\hline & OR & $99 \% \mathrm{CI}$ & OR & $99 \%$ CI \\
\hline \multicolumn{5}{|l|}{ Step 1} \\
\hline Gender $^{\mathrm{a}}$ & 1.03 & {$[0.70,1.50]$} & 1.91 & {$[1.23,2.97]$} \\
\hline Mixed heritage $\mathrm{b}, \mathrm{a}$ & 0.41 & {$[0.26,0.66]$} & 1.77 & {$[1.01,3.09]$} \\
\hline White ${ }^{b}$ & 0.25 & {$[0.12,0.55]$} & 1.05 & {$[0.43,2.58]$} \\
\hline Dwelling & 0.97 & {$[0.54,1.73]$} & 1.03 & {$[0.50,2.11]$} \\
\hline Peri-urban $^{\mathrm{a}}$ & 0.83 & {$[0.51,1.35]$} & 0.39 & {$[0.20,0.75]$} \\
\hline Father employment & 0.86 & {$[0.55,1.34]$} & 0.71 & {$[0.43,1.19]$} \\
\hline \multicolumn{5}{|l|}{ Step 2} \\
\hline Gender ${ }^{\mathrm{a}}$ & 1.07 & {$[0.72,1.59]$} & 2.12 & {$[1.33,3.39]$} \\
\hline Mixed heritage $b, a$ & 0.50 & {$[0.31,0.82]$} & 2.57 & {$[1.40,4.73]$} \\
\hline White $^{\text {b }}$ & 0.28 & {$[0.13,0.64]$} & 1.61 & {$[0.62,4.19]$} \\
\hline Dwelling & 1.05 & {$[0.57,1.92]$} & 0.96 & {$[0.46,2.00]$} \\
\hline Peri-urban ${ }^{\mathrm{a}}$ & 0.89 & {$[0.54,1.45]$} & 0.38 & {$[0.19,0.74]$} \\
\hline Father employment & 0.86 & {$[0.54,1.36]$} & 0.77 & {$[0.45,1.31]$} \\
\hline Indirect home victimisation & 1.20 & {$[0.90,1.59]$} & 1.18 & {$[0.86,1.62]$} \\
\hline Indirect school victimisation & 0.89 & {$[0.68,1.16]$} & 0.87 & {$[0.64,1.18]$} \\
\hline Indirect community victimisation & 0.69 & {$[0.46,1.03]$} & 0.95 & {$[0.60,1.52]$} \\
\hline Direct community victimisation, ${ }^{b}$ & 2.48 & {$[1.26,4.89]$} & 3.76 & {$[1.82,7.76]$} \\
\hline Indirect political victimisation ${ }^{b}$ & 1.05 & {$[1.01,1.10]$} & 1.03 & {$[0.99,1.08]$} \\
\hline
\end{tabular}

Note. $\mathrm{OR}=$ odds ratio.

asignificant variables for suicidal ideation.

$b_{\text {significant variables for hopelessness. }}$ 
The results of the binary logistic regression analyses showed that direct community victimisation (OR: 2.48, $99 \%$ CI: 1.26-4.89) and indirect political victimisation (OR: 1.05, 99 \% CI: 1.01-1.10) were associated with higher odds of hopelessness by 2.48 and 1.05 times, respectively. For suicidal ideation, there was an effect of gender, such that being a girl increased the odds by 2.12 times to have this symptom (OR: 2.12, $99 \%$ CI: 1.33-3.39). Direct community victimisation (OR: 3.76, $99 \%$ CI: 1.82-7.76) was associated with higher odds of suicidal ideation by 3.76 times.

\section{Cumulative Effects of Victimisation on Psychological Functioning}

Results from the hierarchical multiple regression analyses showed the sociodemographic variables in total accounted for $9 \%$ of variance in anxiety, $6 \%$ of variance in depression, and $8 \%$ of variance in perceived stress. Poly-victimisation and its quadratic function accounted for an additional $1 \%, 4 \%$, and $4 \%$ of variance in anxiety, depression, and perceived stress, respectively. In the final model, gender (B's $>.23, p$ 's $<.001$ ) and poly-victimisation (B's $>.09, p$ 's $<.01$ ) were unique predictors for anxiety, depression, and perceived stress. The quadratic function of poly-victimisation was not significantly associated with any psychological symptoms $(p$ 's $>.01)$.

Table 4. Hierarchical regression analyses of sociodemographics and poly-victimisation on psychological functioning in adolescents

\begin{tabular}{|c|c|c|c|c|c|c|}
\hline & \multicolumn{2}{|c|}{ Anxiety } & \multicolumn{2}{|c|}{ Depression } & \multirow{2}{*}{$\begin{array}{c}\text { Perceived } \\
B \\
\end{array}$} & \multirow{2}{*}{$\begin{array}{l}\text { Stress } \\
\Delta \boldsymbol{R}^{2} \\
\end{array}$} \\
\hline & $B$ & $\overline{\Delta R^{2}}$ & $B$ & $\Delta \boldsymbol{R}^{2}$ & & \\
\hline Step 1 & & $.09 * * *$ & & $.06^{* * * *}$ & & $.08^{* * *}$ \\
\hline Gender & $.23 * * *$ & & $.18^{* * *}$ & & $.12 * * *$ & \\
\hline Mixed heritage & $-.17 * * *$ & & $.12^{* *}$ & & $.19^{* * * *}$ & \\
\hline White & $-.19^{* * *}$ & & .00 & & .06 & \\
\hline Dwelling & .02 & & .04 & & $.09 *$ & \\
\hline Peri-urban & -.03 & & $-.07 *$ & & -.06 & \\
\hline Father employment & .02 & & .02 & & .03 & \\
\hline Step 2 & & $.01 *$ & & $.04 * * *$ & & $.04 * * *$ \\
\hline Gender & $.23 * * *$ & & $.18^{* * *}$ & & $.12^{* * * *}$ & \\
\hline Mixed heritage & $-.17 * * *$ & & $.10^{*}$ & & $.16^{* * *}$ & \\
\hline White & $-.18^{* * *}$ & & .02 & & $.07 *$ & \\
\hline Dwelling & .01 & & .02 & & $.08 *$ & \\
\hline Peri-urban & -.04 & & $-.08^{*}$ & & $-.07 *$ & \\
\hline Father employment & .02 & & .03 & & .03 & \\
\hline Poly-victimisation & $.09^{* *}$ & & $.22^{* * *}$ & & $.20^{* * *}$ & \\
\hline $\begin{array}{l}\text { Poly-victimisation } \\
\text { squared }\end{array}$ & $.07 *$ & & .05 & & .01 & \\
\hline
\end{tabular}

The results of the binary logistic regression analyses showed that being a girl was associated with higher odds of suicidal ideation by 2.01 times (OR: 2.01, 99 \% CI: 1.29-3.14). Moreover, poly-victimisation was associated with increased odds of suicidal ideation by 1.04 times (OR: 1.04, 99 \% CI: 1.01-1.08). For hopelessness, there were no significant associations with gender or poly-victimisation ( $p$ 's $>.01)$.

\section{Discussion}

The purpose of the study was to examine the differential effects of violence across major contexts in South African adolescents' lives (home-, school-, community-, political victimisation) as well as the cumulative effects of violence across these contexts (polyvictimisation) on adolescents' levels of hopelessness, anxiety, depression, perceived stress, and suicidal ideation, to provide refined recommendations for mental health interventions in South Africa. The results indicated that victimisation experienced by adolescents in their distal environment, i.e., violence exposure in the community, was associated with increased scores on measures of hopelessness, depression, anxiety, perceived stress, and suicidal ideation. Contrary to our prediction, this pattern was not found for victimisation experienced in their proximal environment, such as home and school. Yet Mrug et al. (2008, 2010) found that violence exposure at home and school are more robust predictors for anxiety and depression than exposure to community victimisation. As they argued, adolescents are likely to know or be in a relationship with the perpetrators of violence in home or school contexts and have limited control over their presence in these contexts, i.e., the closer one's social and relational distance to violence perpetrators, the more adverse the psychological outcomes could be. Our findings, however, corroborate several studies conducted in South Africa that demonstrate community victimisation as the most consistent predictor for maladaptive psychological outcomes (e.g., Barbarin \& Richter, 2001; Kaminer et al., 2013). This consistent association may be related to the environmental context in South Africa. South Africa is unique in the sense that the prevalence of violence in the general public is high, which supports our finding on the association between psychological functioning and community victimisation, and also sheds light on a possible cross-cultural variation in violence exposure and its associated psychological consequences due to contextual differences. Adolescents living in violent communities may feel continually at risk for victimisation by acts that are unforeseeable (e.g., robberies, gang activities, shooting) and not under their control, and as a result may experience a sense of fear for their own safety and the safety of the people around them. This may lead to chronic emotional hyperarousal which is associated with adverse mental health consequences (Fowler, Tompsett, Braciszewski, Jacques-Tiura, \& Baltes, 2009). 
Table 5. Binary regression analyses of sociodemographics and poly-victimisation on psychological functioning in adolescents

\begin{tabular}{|c|c|c|c|c|}
\hline & \multicolumn{2}{|c|}{ Hopelessness } & \multicolumn{2}{|c|}{ Suicidal Ideation } \\
\hline & OR & $99 \%$ CI & OR & $99 \%$ CI \\
\hline \multicolumn{5}{|l|}{ Step 1} \\
\hline Gender $^{\mathrm{a}}$ & 1.01 & {$[0.70,1.47]$} & 1.95 & {$[1.26,3.01]$} \\
\hline Mixed heritage $\mathrm{b}, \mathrm{a}$ & 0.40 & {$[0.25,0.63]$} & 1.78 & {$[1.03,3.06]$} \\
\hline White ${ }^{b}$ & 0.24 & {$[0.11,0.51]$} & 1.02 & {$[0.42,2.46]$} \\
\hline Dwelling & 0.94 & {$[0.54,1.67]$} & 1.00 & {$[0.50,2.01]$} \\
\hline Peri-urban ${ }^{\mathrm{a}}$ & 0.82 & {$[0.51,1.31]$} & 0.36 & {$[0.19,0.70]$} \\
\hline Father employment & 0.83 & {$[0.53,1.28]$} & 0.71 & {$[0.43,1.17]$} \\
\hline \multicolumn{5}{|l|}{ Step 2} \\
\hline Gender $^{\mathrm{a}}$ & 1.03 & {$[0.71,1.49]$} & 2.01 & {$[1.29,3.14]$} \\
\hline Mixed heritage $\mathrm{b}^{\mathrm{b}, \mathrm{a}}$ & 0.41 & {$[0.26,0.65]$} & 1.72 & {$[0.99,2.99]$} \\
\hline White ${ }^{b}$ & 0.24 & {$[0.11,0.52]$} & 1.12 & {$[0.46,2.74]$} \\
\hline Dwelling & 0.96 & {$[0.54,1.70]$} & 0.97 & {$[0.48,1.95]$} \\
\hline Peri-urban ${ }^{\mathrm{a}}$ & 0.82 & {$[0.51,1.32]$} & 0.35 & {$[0.18,0.68]$} \\
\hline Father employment & 0.83 & {$[0.54,1.29]$} & 0.74 & {$[0.44,1.22]$} \\
\hline Poly-victimisation ${ }^{\mathrm{a}}$ & 1.00 & {$[0.97,1.03]$} & 1.04 & {$[1.01,1.08]$} \\
\hline Poly-victimisation squared & 1.00 & {$[1.00,1.00]$} & 1.00 & {$[1.00,1.00]$} \\
\hline
\end{tabular}

\section{Note. $\mathrm{OR}=$ odds ratio.}

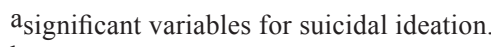

$\mathrm{b}_{\text {significant variables for hopelessness. }}$

We found that adolescents who had exposure to political victimisation - hearing about Apartheid-related victimisation of others - reported anxiety and feelings of hopelessness, suggesting that Apartheid continues to have a negative psychological impact on the younger South African generations. The transmission of trauma to future generations, or intergenerational trauma, has been documented in the context of ethno-political violence and has implications for psychological adjustment in youth (Bombay, Matheson, \& Anisman, 2009; Kahane-Nissenbaum, 2011). Mechanisms by which the indirect exposure to political violence of Apartheid affect the psychological functioning of South African adolescents require further exploration since the current data prohibit drawing firm conclusions. Song et al. (2014) found parents/caregivers exposed to political victimisation can negatively influence the psychological functioning of their children as a result of their own mental distress, maladaptive behaviours, and poor parenting skills.
Our bivariate correlation analyses showed all types of victimisation were positively intercorrelated, indicating an overlap in the experience of victimisation. Indeed, victimisation is rarely a one-off event, but a condition of ongoing danger across multiple life domains that adolescents experience (Finkelhor et al., 2007; Kaminer et al., 2013), i.e., poly-victimisation. Several studies in South Africa (Leoschut \& Kafaar, 2017; Kaminer et al., 2013) found poly-victimisation is a common experience for adolescents. In line with our prediction and other studies (Finkelhor et al., 2007; Latsch et al., 2017; Mrug et al., 2008; Soler et al., 2012), our results showed a cumulative impact of violence, and that the levels of anxiety, depression, perceived stress, and suicidal ideation in adolescents were higher as victimisation experiences increased. The adverse psychological effects of polyvictimisation may be due to the context of multiple and continuous violence exposure across major domains in an adolescent's life that interferes with adaptive coping (Finkelhor et al., 2007). Poly-victimisation across multiple contexts results in relatively few areas where one can feel safe, and may undermine healthy development and elicit feelings of insecurity, posing a unique set of psychological challenges for adolescents (Mrug et al., 2008).

Moreover, poly-victimisation may have more deleterious psychological effects than other forms of (singular) violence exposure (Latsch et al., 2017). Finkelhor et al. (2007) showed polyvictimisation had higher predictive power on anxiety and depression symptoms in children than physical assault or sexual assault alone. In addition, they found poly-victimisation was a stronger predictor for anxiety than exposure to a single type of victimisation and repeated victimisation of the same type (chronic victimisation). Similarly, Gustafsson et al. (2009) found an additive count of multiple traumatic events (e.g., car accident, victim of robbery, witness to someone getting hurt) to be a consistent and more significant predictor for trauma symptoms in children and adolescents than any single traumatic event. Thus, in line with these studies, the current research adds to the literature by demonstrating the cumulative and interactive effects among different types of victimisation, and its damaging and potentially less reversible psychological effects of poly-victimisation on young people. Interestingly, in contrast to what we expected, there were no curvilinear relationships between violence exposure and psychological symptoms. In the current sample, there is a linear relationship between poly-victimisation and psychological symptoms - each additional victimisation increases the likelihood of maladjustment for these adolescents, which in the context of South Africa is cause for concern. Future studies may help the understanding of curvilinear relationships between violence and psychological functioning, as some researchers have demonstrated such a relationship (e.g., Morales \& Guerra, 2006; Mrug et al., 2008). These curvilinear effects are possibly linked to a function of adolescents' psychological desensitization, or coping and resilience in the face of cumulative risks and adversities (Fergus \& Zimmerman, 2005; Folkman, Lazarus, Dunkel-Schetter, DeLongis, \& Gruen, 1986; Mrug et al., 2008). 
Of the sociodemographic variables we investigated, only gender showed a consistent association with most psychological symptoms. Girls were more likely than boys to experience depression, anxiety, perceived stress, and suicidal ideation. Globally, more than a third of women (35\%) are victims of physical or sexual violence and are prone to mental problems (Garcia-Moreno et al., 2013). The high magnitude of violence towards women is also evident in South Africa. In a recent Demographic and Health Survey, 21\% of women had ever experienced physical violence by a partner, and $6 \%$ had ever experienced sexual violence by a partner (Statistics South Africa, 2016). The rate of murder of women by intimate partners in South Africa is six times the global average (Seedat, Van Niekerk, Jewkes, Suffla, \& Ratele, 2009). These data demonstrate that being female in South Africa is subjected to high levels of violence, which poses a significant risk for psychological problems in women of all ages. Furthermore, our results showed that boys and girls confronted different types of violence. Specifically, girls reported more indirect home victimisation than boys, and boys reported more indirect- community and school victimisation than girls. However, the effect of gender on psychological functioning in our study was not influenced by any of these victimisation types, as no significant interactions between gender and violence were found. This means that being a girl entails a higher risk for experiencing adverse psychological functioning, irrespective of the levels of these types of exposure to violence. The lack of gender by violence moderation effects on psychological symptoms has also been noted in another South African study (Kaminer et al., 2013).

\section{Limitations and Directions for Future Research}

Although this study delineated the differential and cumulative effects of violence victimisation on psychological functioning in a representative sample of adolescents in the Western Cape Province, several limitations should be noted. First, the cross-sectional design and self-reported data prevent drawing causal conclusions. Longitudinal research would be useful to understand the causal links of violence and its associated psychological correlates in adolescents. It may also be advantageous for future research to control for factors related to psychological functioning in adolescents, such as non-victimisation traumas (e.g., illnesses, accidents, natural disasters) and chronic adversities (e.g., substance abuse by family members, repeating school) (Finkelhor et al., 2007). Moreover, factors that may exacerbate or alleviate the relationship between victimisation and psychological functioning of adolescents, such as coping skills (e.g., Horwitz, Hill, \& King, 2011), emotion regulation (e.g., Flouri \& Mavroveli, 2013), self-esteem (e.g., Turner, Shattuck, Finkelhor, \& Hamby, 2017), social support (e.g., Leshem, Haj-Yahia, \& Guterman, 2016), and caregiver factors (e.g., Guttmann-Steinmetz, Shoshani, Farhan, Aliman, \& Hirschberger, 2012) can be taken along in future studies to gain a holistic view of the mechanism of violence.
In addition, the victimisation measures used in the current research may have been suboptimal. We used an additive count for poly-victimisation, thus data on the intensity and frequency of exposure to each type of victimisation are lost - although this approach is deemed appropriate in an exploratory stage of work on multi-victimisation measurement (Finkelhor et al., 2007). Furthermore, the limited number of items for home and school victimisation might have lowered the reliability of these variables due to the fact that only two victimisation constructs, i.e., "beaten" and "chased", were measured. This may have attenuated the real-life impact of home and school victimisation in South African adolescents, because other types of victimisation such as peer or sibling assault, physical or emotional bullying, neglect, verbal sexual harassment can also occur at home and school (Finkelhor et al., 2007).

Lastly, community victimisation was differentiated into indirect and direct exposure, whereas the victimisation variables in other contexts were not. This differentiation seems relevant, since several South African studies have shown differential psychological effects of indirect and direct victimisation across multiple contexts, in which indirect community victimisation is consistently related to adjustment problems in youth compared to indirect or direct exposure in other contexts (e.g., Barbarin \& Richter, 2001; Shields, Nadasen, \& Pierce, 2009). Future research may consider examining both indirect and direct victimisation across important contexts in adolescents' lives to enhance the understanding of the effects of different types of victimisation and provide more refined recommendations to alleviate these effects.

\section{Clinical and Policy Implications}

The results of the study suggest the need for gender-specific mental health interventions to promote healthy psychological functioning for girls, as we found them vulnerable to psychological problems. For example, a gender-responsive programme that involves a sensitive approach with an understanding to the realities of women's lives is able to provide a safe, trusting, and supportive environment to reduce their mental health symptoms and substance use (Covington, Burke, Keaton, \& Norcott, 2008). Moreover, our finding on the main effect of community victimisation on adolescent psychological functioning stresses the importance of the integration of various service providers in the community such as the South African Police Service, local non-governmental organizations, and local municipal authorities to create a safe environment and actively respond to the needs of adolescents exposed to violence in the community. For example, a social development center with collective efforts on the part of a multidisciplinary team (e.g., community leaders, social workers, psychologists) may help improve victims' access to mental health care resources (Schmidt, 2014). Indeed, previous research has established that a caring and cohesive community protects youth victims against an increase in vulnerability for psychological 
distress over time (Jain, Buka, Subramanian, \& Molnar, 2012). In addition, there is also a need to incorporate an understanding of intergenerational transmission of trauma in mainstream interventions to foster healing in the South African younger generations who may be adversely affected by knowing about others' victimisation experiences of the political violence of Apartheid (Roy, Noormohamed, Henderson, \& Thurston, 2015).

Furthermore, our study revealed violence exposure in one ecological context to be positively associated with exposure in other contexts, and exposure to poly-victimisation yielded significant psychological problems in adolescents. We recommend practitioners and policy makers in South Africa implement multi-faceted and multi-purpose mental health programmes that involve active identification and referral, health education, support, counselling, and follow up, as well as to consider the full spectrum of sources of violence across major contexts of an adolescent's life in order to gain a comprehensive profile of their victimisation experience. A school-based intervention may be useful as adolescents spend significant amount of time in school, thus making school a key platform to provide support for healthy functioning. A study found that a socioeconomically disadvantaged sample of children who had exposure to violence had lower scores in symptoms of trauma and depression than the wait-list group, three months after attending a school-based cognitive behavioural group therapy (Stein, Jaycox, Kataoka, Wong, Tu, Elliott, \& Fink, 2003). The therapy consisted of 10 sessions aimed at combating negative thoughts, and enhancing skills in adaptive coping and problem solving in these children (Stein et al., 2003). Moreover, a school-family programme where school personnel provide parenting education to facilitate parental support to victimized youth may extend the impact across multiple contexts to build adaptive emotional and behavioural coping skills in adolescents (Brubaker, Brubaker, \& Link, 2001; Kliewer et al., 2004), both as a means to prevent adverse psychological symptoms and to strengthen the resilience to violence.

\section{Notes}

'A former system of institutionalised racial segregation in South Africa $(1948$ - 1994) in which the access to economic resources, medical care, and educational and employment opportunity were restricted for people socially classified as "non-white".

${ }^{2}$ Formal dwelling type refers to a brick house or an apartment/flat; informal dwelling type refers to a shack, hut, house made of mud and stick, or house made of mud bricks and thatch. 


\title{
Chapter 5
}

\author{
Exposure to Violence across \\ Multiple Contexts and Health \\ Risk Behaviours in South \\ African Adolescents: \\ The Moderating Role of \\ Emotion Dysregulation
}




\section{Abstract}

The association between violence exposure and health risk behaviours in South African adolescents, and the moderating role of emotion dysregulation were investigated. A multi-ethnic sample of adolescents $(n=925:$ boy: $47.3 \%$, girl: $52.7 \%, M$ age $=16$ years, $S D=1.54$ ) completed a survey. Violence exposure across different contexts (home-, school-, community-, political victimisation), emotion dysregulation (inability to regulate sadness and anger), and a composite measure of health risk behaviours (smoking, substance use, risky sexual behaviour) were examined. Boys reported more risk behaviours than girls, $t(844)=5.25, p<.001$. Direct community victimisation was a predictor for boys' risk behaviours, $B=.22, p<.001$. Indirect school victimisation and direct community victimisation were predictors for girls' risk behaviours, $B$ 's $=.19$, $p$ 's $<.01$. Girls reported higher emotion dysregulation than boys, $t(748)=-2.95, p<.01$. Only for girls, emotion dysregulation moderated the associations of indirect home victimisation, $B=16, p<.01$, and direct community victimisation, $B=15, p<.05$, with risk behaviours. Interventions may target emotion regulation skills, particularly for girls, to enhance resilience to the negative effects of violence on behaviours.
South Africa is disproportionately affected by violence. Although the political violence of Apartheid ${ }^{1}$ has subsided by 1994 , interpersonal violence is still prominent among the general public. On average, 52 murders, 109 rapes, and 470 physical assaults are reported daily (South African Police Service, 2016) and the mortality resulting from interpersonal violence is over seven times the global rate (Norman, Matzopoulos, Groenewald, \& Bradshaw, 2007). Due to the country's high prevalence of violence, both indirect (i.e., witnessing or hearing) and direct violence victimisation (i.e., personal experience) are common among South African adolescents (Shields, Nadasen, \& Pierce, 2009). In particular, South African adolescents are exposed to violence across major developmental domains in their lives, including home- (e.g., physical violence between parents), school- (e.g., bullying among learners), and community violence (e.g., robbery or assault) (Kaminer, du Plessis, Hardy, $\&$ Benjamin, 2013). For example, in a representative sample in the Western Cape Province of South Africa, almost all adolescents (90\%) had witnessed community violence, $40 \%$ had been a direct victim of community violence, $77 \%$ had witnessed home violence, $59 \%$ had been a direct victim of home violence, and $76 \%$ reported either direct or indirect exposure to school violence (Kaminer et al., 2013). Given the unique historic context in South Africa, the younger generations may have also heard stories of the brutality and discrimination that happened during Apartheid, against their relatives or people they know (Sui et al., 2018).

Exposure to violence has been associated with a range of adverse psychological and behavioural outcomes in adolescents, including anxiety, depression, trauma symptoms, aggression, and substance use (Bach \& Louw, 2010; Finkelhor, Ormrod, \& Turner, 2007; Mrug, Loosier, \& Windle, 2008; Sui et al., 2018). It is crucial to examine the effects of violence on adolescents to inform interventions, as maladjustments during this developmental period can have a significant impact on their education and economic outcomes, psychosocial functioning, and health in adulthood (Botticello, 2009; Das-Munshi et al., 2016).

\section{Violence Exposure and Health Risk Behaviours}

Typically, adolescents are sensation and novelty seeking, and have a propensity for risk behaviours (Steinberg, 2007). Very often, these behaviours can affect health and contribute to the leading causes of mortality and morbidity among adolescents, i.e., health risk behaviours (El Achhab et al., 2016). Examples of health risk behaviours include (but are not limited to) alcohol or drug intoxication, smoking, and risky sexual behaviours, which place adolescents at risk for a range of chronic and infectious diseases (Reddy et al., 2013). Health risk behaviours during adolescence can be particularly problematic because they are associated with adverse short- and long-term psychosocial consequences, including early pregnancy, risk for HIV/AIDS, as well as alcohol dependency or abuse, and criminal behaviours in adulthood (Brown et al., 2012; Hessler \& Katz, 2010). Moreover, health risk behaviours tend to cluster and the majority of adolescents engage in multiple of these 
behaviours (e.g., both alcohol and drug use; Bobrowski, Czabala, \& Brykczynska, 2007; Coleman, Wileyto, Lenhart, \& Patterson, 2014; MacArthur et al., 2012).

Violence exposure across different contexts can foster health risk behaviours in adolescents. For example, witnessing community and home violence, and being a direct victim of community violence are significantly and positively related to alcohol and drug use (Begle et al., 2011; Lee, 2012; Moreira et al., 2008). Being a victim of community violence is associated with increased risky sexual behaviours, such as having multiple sexual partners, a high frequency of sexual activity, and having sex without condoms (Albus, Weist, \& Perez-Smith, 2004; Voisin, Jenkins, \& Takahashi, 2011). Exposure to political violence, or violence motivated by political reasons, is linked to alcohol, cannabis, and ecstasy use (Schiff et al., 2012). The associations between violence exposure and different types of health risk behaviours have also been documented in several South African studies (Brook, Morojele, Pahl, \& Brook, 2006; Brook, Rubenstone, Zhang, Morojele, \& Brook, 2011; Morojele \& Brook, 2006), emphasising that violence is one of the major environmental stressors that can adversely influence adolescent health related behaviours.

\section{Violence and Emotion Dysregulation}

Studies have consistently shown that exposure to violence, such as living in a violent community (Sharkey, Tirado-Strayer, Papachristos, \& Raver, 2012), and being a victim of community violence (Kelly, Schwartz, Gorman, \& Nakamoto, 2008) or childhood maltreatment (Kim \& Cicchetti, 2010) are associated with diminished emotion regulation capacities in youth, or emotion dysregulation. Emotion dysregulation is defined as the impaired ability to regulate and tolerate negative emotional states (Dvir, Ford, Hill, \& Frazier, 2014). Encounters with violence are inherently stressful and may produce heightened emotional arousal (Mrug, Looiser, \& Windle, 2010); typically, emotions of sadness and anger (Kliewer et al., 2004, 2017). An adolescent's perceived capacity to control emotional arousal and to adaptively cope with sadness and anger reflects an important aspect of emotion regulation during this developmental period (Zeman, Shipman, \& Suveg, 2002).

\section{Emotion Dysregulation and Health Risk Behaviours}

Researchers suggest that emotion regulation capacity is a key factor to overcome the adversities that put young people at risk for maladjustments (Smokowski, Mann, Reynolds, \& Fraser; 2004; Masten \& Coatsworth, 1998). However, many adolescents may not be adequately equipped to regulate their emotions, since the structures involved in emotion regulation are among the last to mature in the developing brain (Bell \& McBride, 2010). Furthermore, adolescence is a period of transition characterised by significant increases in emotional reactivity and greater sensitivity to stressors (Casey et al., 2010). As such, negative affect is likely to emerge frequently during this period and adolescents are vulnerable to emotion dysregulation (McLaughlin, Hatzenbuehler, \& Hilt, 2009). Emotion dysregulation is predictive of the onset of a variety of health risk behaviours in youth, including risky sexual behaviours, cigarette smoking, and alcohol and drug use (Bell \& McBride, 2010; Brown et al., 2012; Hessler \& Katz, 2010; Wills, Walker, Mendoza, \& Ainette, 2006; Wills, Simons, Sussman, \& Knight, 2016).

\section{Emotion Dysregulation as a Moderator}

It is worth noting that not all young people exposed to violence develop behavioural problems (Moffitt et al., 2013). Prior research has indicated that violence exposure and emotion dysregulation are each associated with an increased likelihood of health risk behaviours among adolescents (e.g., Voisin et al., 2011; Wills et al., 2016). According to the diathesis-stress model (Spielman, Caruso, \& Glovinsky, 1987), negative health outcomes are a result of the interaction between an individual's predispositional vulnerability and an environmental stressor. This suggests variations in the strength of the relationship between violence exposure and one's behaviour, and that some adolescents may be more likely to develop health risk behaviours if a certain predisposed vulnerability (e.g., psychological problem) is present in addition to the external stressor, i.e., violence exposure. Hence, emotion dysregulation may be a vulnerability factor that aggravates the negative effects of violence, placing adolescents at risk for engaging in health risk behaviours.

Studies have demonstrated the negative influence of emotion dysregulation on youth development. Emotion dysregulation, particularly difficulty in managing sadness and anger, is associated with behavioural problems such as peer-rated physical and relational aggression (Zeman, Shipman, \& Penza-Clyve, 2001; Zeman et al., 2002). Emotion dysregulation has also been found to diminish the effectiveness of children's coping responses to peer victimisation (Cooley, 2019). Furthermore, Kliewer et al. (2017) found that emotion dysregulation can exacerbate the effects of cumulative psychosocial risks (e.g., maternal mental health problems, family stress, and violence exposure) on youth internalising and externalising problems. Although empirical associations between violence, emotion dysregulation, and health risk behaviours have been found in the literature, researchers have not yet extended this work to the moderating role of emotion dysregulation in the relationship between violence exposure across different contexts and health risk behaviours in adolescents. Since adolescence presents a time of thrill-seeking as well as important changes in affective experience and regulation, research on the moderating role of emotion dysregulation on health risk behaviours in South African adolescents exposed to violence may be particularly relevant for youth intervention development. 


\section{The Role of Gender}

Research has suggested a pattern of gender difference in health risk behaviours in adolescents exposed to violence, such that boys are more likely than girls to use alcohol and drugs when exposed to direct and indirect community victimisation and physical assault (Begle et al., 2011; Moreira et al., 2008). Gender differences have also been noted in the developmental outcomes during adolescence in general, such that boys are more likely than girls to smoke cigarettes, use drugs, drink alcohol, and engage in risky sexual behaviours (Croisant, Laz, Rahman, \& Berenson, 2013; Mennis, \& Mason, 2012; Slone \& Mayer, 2015), whereas girls are more prone to internalising symptoms such as anxiety, depression (Mrug et al., 2008; Slone \& Mayer, 2015), perceived stress in life, and suicide ideation (Sui et al., 2018). Girls also have greater difficulties regulating negative emotions than their male counterparts (Bender, Reinholdt-Dunne, Esbjorn, \& Pons, 2012). Given these differences between boys and girls, we focus on the role of gender in the present study as well.

\section{This Study}

There is increasing evidence that indirect and direct violence exposures are associated with health risk behaviours in adolescents. However, there is no research yet that has simultaneously examined the differential effects of violence across multiple contexts (home-, school-, community-, and political victimisation due to Apartheid) on health risk behaviours (smoking, alcohol use, soft drug use, hard drug use, and risky sexual behaviour) in South African adolescents, the moderating role of emotion dysregulation in these associations, and the role of gender. In the current research, we examine these associations in a representative sample of South African adolescents in the Western Cape Province. The results of the study may inform the development of behavioural interventions to reduce health risk behaviours in this vulnerable population of adolescents exposed to violence in South Africa.

\section{Method}

\section{Participants}

Participants were drawn from the national sample of adolescents that completed the third South African Youth Risk Behaviour Survey (YRBS; Reddy et al., 2013). A two-stage cluster sampling procedure was used to recruit participants (see also Kann et al., 2016). The current study comprised of a random sample of adolescents in public secondary schools in the Western Cape Province of South Africa. The original sample consisted of 1300 adolescents from Grade 8 to 11 (boy: $45.9 \%$; girl: $54.1 \%$ ). Since the focus of our study is on health risk behaviours as an outcome of violence exposure, thus, for the analyses, we selected the majority of the adolescents $(71.2 \%)$ who reported at least one health risk behaviour, making the final sample to be 925 adolescents (boy: $47.3 \%$; girl:
$52.7 \%)$. Their ages range from 13 to 22 years $(M=16$ years for both boys and girls, $S D=1.58$ and 1.49 , respectively).

\section{Procedure}

Participants completed the third South African YRBS (Reddy et al., 2013) that examined the national prevalence of adolescent risk behaviours related to infectious disease (e.g., sexual activity), chronic disease (e.g., physical activity), injury and trauma (e.g., traffic safety), and mental health (e.g., substance use). For the purpose of this study, the data on substance use and sexual activity - specifically, smoking, alcohol use, soft drug use, hard drug use, and risky sexual behaviour - of adolescents in the Western Cape Province of South Africa were analysed. In addition to the questions of the YRBS, these participants responded to another set of items related to violence exposure across different contexts, namely, home-based, school-based, community-based victimisation, and political victimisation of Apartheid. For those who were under the age of 18 years, parental consents were required along with the assent forms. The participants were told to bring their signed assent and consent forms to school the next day and the ones who agreed to participate were invited to complete the questionnaire in the classrooms.

\section{Measures}

\section{Indirect Home and School Victimisation}

Indirect exposure to home and school violence was measured by two items adapted from the Survey of Children's Exposure to Violence (Richters \& Saltzman, 1990) which were phrased slightly different for each type of violence exposure: 'How many times in your whole life have you seen people chased by someone who wanted to hurt them in your home/ in your school?' and 'How many times in your whole life have you seen people beaten up by someone in your home/in your school?' The responses ranged on a scale from 1 (never) to 4 (many times), and were averaged to form an index of indirect victimisation for each context. Higher scores indicate higher frequency of indirect exposure to violence at home $(r=.40)$ and at school $(r=.47)$.

\section{Indirect and Direct Community Victimisation}

Both direct and indirect exposure to community violence were measured by fifteen items adapted from the Survey of Children's Exposure to Violence (Richters \& Saltzman, 1990) to suit the South African context. For direct victimisation, the adolescents were asked about being a victim of physical violence, threats, and interpersonal crime in the community in life time; whereas for indirect community violence exposure, the items were about whether they had witnessed or heard about these same types of violence. Example questions are: 'How many times in your whole life have you seen people robbed or mugged by someone?', '...have you been seriously wounded by someone?' Items were averaged, with higher 
scores indicating more exposure to direct community victimisation $(\alpha=.84)$ and indirect community victimisation $(\alpha=.90)$

\section{Indirect Political Victimisation}

The indirect political victimisation scale was adapted from a previous study (Whitbeck, Hoyt, McMorris, Chen, \& Stubben, 2001) to measure whether adolescents had heard or knew of any of South Africa's colonial and historical events of Apartheid that happened to someone in their lives, such as a family, a friend or an acquaintance. The scale has twenty-eight items related to political violence (e.g., forced removal, experience of being imprisoned, and incidents of racial discrimination). Example items are: 'Have any of your family/friends or anyone you know ever been refused entry into a public place or area because of their race?', '... ever been sent to prison or detained because of political views that opposed the government?' The response options were 'yes', 'no', and 'I don't know'. The 'yes' responses were coded as 1 and summed to obtain an index of indirect exposure to political violence (possible range $0-28 ; \alpha=.83$ ).

\section{Health Risk Behaviours}

Five types of health risk behaviours were measured in the YRBS (Reddy et al., 2013): smoking, alcohol use, soft drug use, hard drug use, and risky sexual behaviour. Smoking was measured by the frequency of recent cigarette use on a scale from 1 ( 0 days) to 7 (all 30 days). Alcohol use was measured by the frequency of recent use of alcohol and binge drinking, both on a scale from 1 (never) to 5 (very often). Soft drug use was measured by the frequency of recent marijuana use on a scale from 1 (never) to 5 (very often). Hard drug use was measured by the frequency of life time use of nine types of illegal drugs on a scale from 1 (never) to 5 (very often) that are relevant in the South African contexts, including glue (sniffed), TIK, Mandrax, cocaine, heroin, Whoonga, drug via needle injection, overthe-counter or prescription drugs (e.g., pain killers, cough mixtures, and diet pills), and any hallucinant drugs (e.g., ecstasy, LSD, speed, or magic mushrooms). Risky sexual behaviour was measured by the frequency of condom use on a scale from 1 (I have never had sex) to 6 (we always use a condom).

Since the scales differed among behaviours and the proportions of the participants who reported each of these health risky behaviours varied between $21.5 \%$ and $51.6 \%$ (see Table 1), it was decided to dichotomise each risk behaviour such that $0=$ no such behaviour at all and 1 =performed the risk behaviour (once or more than once). We then summed the number of risk behaviours and selected the participants with at least one risk behaviour for the analyses, resulting in a composite risk behaviour scale with scores ranging from 1 (one risk behaviour) to 5 (five risk behaviours) $(M=2.01, S D=1.10, \alpha=.55)$.
Table 1. Proportions of adolescents with and without health risk behaviours

\begin{tabular}{lcccccc}
\hline & \multicolumn{2}{c}{ Yes } & & \multicolumn{2}{c}{ No } \\
\cline { 2 - 3 } \cline { 6 - 7 } Health Risk behaviour & $\mathbf{N}$ & $\mathbf{\%}$ & & $\mathbf{N}$ & $\mathbf{\%}$ \\
\hline Smoking & 406 & 28.7 & & 1009 & 71.3 \\
Alcohol use & 712 & 51.6 & & 668 & 48.4 \\
Soft drug use & 214 & 15.2 & & 1197 & 84.8 \\
Hard drug use & 295 & 21.5 & & 1074 & 78.5 \\
Risky sexual behaviour & 383 & 28.4 & & 967 & 71.6 \\
\hline
\end{tabular}

\section{Emotion Dysregulation}

Participants' emotion dysregulation was measured by the anger and sadness dysregulation subscales (10 items) of the Children's Emotion Management Scales (CEMS; Zeman et al., 2002). Example questions are: 'I do things like slam doors when I am angry', 'I wine/fuss about what is making me sad'. Responses ranged from 1 (never) to 5 (always). Scores were averaged, and higher scores indicate higher levels of emotion dysregulation $(\alpha=.76)$

\section{Data Analysis}

Data were analysed using SPSS version 23. Significance was set at $\alpha=.05$. Pearson correlation coefficients were examined to understand the intercorrelations among all the variables. Independent sampled $t$-tests were conducted to investigate the gender differences in the levels of violence exposure, emotion dysregulation, and health risk behaviours. Next, hierarchical multiple regressions were conducted separately for boys and girls to understand the associations between violence exposure across different contexts and health risk behaviours, as well as the moderating role of emotion dysregulation in these associations. For both gender groups, age was entered in step 1, the five types of victimisations and emotion dysregulation were entered as main effects in step 2, and the product terms of each type of victimisation with emotion dysregulation were entered in step 3 . All variables were first centred before the interactions were computed to reduce multicollinearity (Aiken \& West, 1991).

\section{Results}

Table 2 shows the intercorrelations of victimisation across different contexts, emotion dysregulation, and the composite measure of health risk behaviours. Age was positively associated with risk behaviours $(r=.18 ; p<.01)$. Being a boy was associated with more exposure to indirect school victimisation and direct community victimisation $(r=-.12$ and -.10 , respectively; $p$ 's $<.01$ ). Being a girl was associated with higher emotion dysregulation 
$(r=.11 ; p<.01)$, whereas being a boy was associated with more health risk behaviours ( $r=$ $-.17 ; p$ 's $<.01)$. Indirect school victimisation, indirect community victimisation, and direct community victimisation were positively associated with health risk behaviours $(r$ 's $=.12$ to .27; $p$ 's $<.01)$. Higher levels of victimisation across different contexts were associated with more emotion dysregulation ( $r$ 's $=.08$ to $.14 ; p$ 's $<.01$ ), except for indirect political victimisation $(r=-.14 ; p<.01)$. Emotion dysregulation was not associated with health risk behaviours $(p>.05)$.

Table 2. Intercorrelations, means, and standard deviations for all variables

\begin{tabular}{lccccccccccc}
\hline & $\boldsymbol{M}$ & $\boldsymbol{S D}$ & $\mathbf{1}$ & $\mathbf{2}$ & $\mathbf{3}$ & $\mathbf{4}$ & $\mathbf{5}$ & $\mathbf{6}$ & $\mathbf{7}$ & $\mathbf{8}$ & $\mathbf{9}$ \\
\hline 1. Gender & & 1.53 & .50 & - & & & & & & & \\
2. Age & 16.16 & 1.54 & $-.09^{*}$ & - & & & & & & \\
3. Indirect home victimisation & 1.67 & .77 & .03 & .05 & - & & & & & \\
4. Indirect school victimisation & 2.44 & .89 & $-.12^{* *}$ & .03 & $.29^{* *}$ & - & & & & \\
5. Indirect community victimisation & 2.41 & .63 & -.06 & .05 & $.30^{* *}$ & $.56^{* *}$ & - & & & \\
6. Direct community victimisation & 1.45 & .39 & $-.10^{* *}$ & .07 & $.37^{* *}$ & $.25^{* *}$ & $.40^{* *}$ & - & & & \\
7. Indirect political victimisation & 4.62 & 5.47 & -.02 & -.02 & $.13^{* *}$ & .07 & $.18^{* *}$ & $.28^{* *}$ & - & & \\
8. Health risk behaviours & 2.01 & 1.10 & $-.17^{* *}$ & $.18^{* *}$ & .06 & $.14^{* *}$ & $.12^{* *}$ & $.27^{* *}$ & .06 & - & \\
9. Dysregulation & 2.34 & .71 & $.11^{* *}$ & -.05 & $.14^{* *}$ & $.10^{* *}$ & $.08^{*}$ & $.12^{* *}$ & $-.14^{* *}$ & .04 & - \\
\hline
\end{tabular}

${ }^{a} 1=$ boy, $2=$ girl; $* * p<.01,{ }^{*} p<.05$

When compared to girls, boys reported more exposure to indirect school victimisation, $t$ $(739)=3.28, p<.001$, and direct community victimisation, $t(712)=2.83, p<.01$. No gender differences were found for other victimisation scales. Girls reported more emotion dysregulation than boys $t(748)=-2.95, p<.01$. Boys were more likely than girls to engage in health risk behaviours $t(844)=5.25, p<.001$.

\section{Exposure to Violence and Emotion Dysregulation on Health Risk}

\section{Behaviour}

Standardized regression coefficients and changes in $R^{2}$ values from the multiple regressions are presented in Table 3 for boys and girls.

Boys. For boys, age accounted for $5 \%$ of the variance in health risk behaviours. When the five types of victimisation and emotion dysregulation were added, the variables together accounted for an additional $6 \%$ of the variance. In the final model, the change in variance was not significant. Age and direct community victimisation were unique predictors for health risk behaviours (both $\mathrm{B}$ 's $=.22, p$ 's $<.001$ ).
Girls. For girls, age was not a significant predictor. When the five types of victimisation and emotion dysregulation were added in the model, the variables together accounted for an additional $9 \%$ of the variance. In the final model, the interactions accounted for an additional $5 \%$ of the variance. The final model showed that indirect school victimisation and direct community victimisation were unique predictors for health risk behaviours (Both B's $=.19$, $p$ ' $\mathrm{s}<.01)$. The interaction of indirect home victimisation and emotion dysregulation $(\mathrm{B}=16$, $p<.01)$, and the interaction of direct community victimisation and emotion dysregulation $(\mathrm{B}=15, p<.05)$ significantly predicted health risk behaviours.

Table 3. Hierarchical multiple regression of the effects of exposure to violence and emotion dysregulation on health risk behaviours in adolescents

\begin{tabular}{|c|c|c|c|c|}
\hline & \multicolumn{2}{|c|}{ Boys } & \multicolumn{2}{|c|}{ Girls } \\
\hline & $B$ & $\Delta R^{2}$ & $B$ & $\Delta R^{2}$ \\
\hline Step 1 & & $.05 * * *$ & & .01 \\
\hline Age & $.22 * * *$ & & .08 & \\
\hline Step 2 & & $.06^{* *}$ & & $.09 * * *$ \\
\hline Age & $.22 * * *$ & & .04 & \\
\hline Indirect home victimisation & -.02 & & -.07 & \\
\hline Indirect school victimisation & -.05 & & $.21 * * *$ & \\
\hline Indirect community victimisation & .05 & & -.05 & \\
\hline Direct community victimisation & $.24 * * *$ & & $.22 * * *$ & \\
\hline Indirect political victimisation & -.01 & & -.02 & \\
\hline Emotion dysregulation & -.01 & & .08 & \\
\hline Step 3 & & .02 & & $.05 * * *$ \\
\hline Age & $.22 * * *$ & & .04 & \\
\hline Indirect home victimisation & -.04 & & -.10 & \\
\hline Indirect school victimisation & -.06 & & $.19^{* *}$ & \\
\hline Indirect community victimisation & .06 & & -.04 & \\
\hline Direct community victimisation & $.22 * * *$ & & $.19 * *$ & \\
\hline Indirect political victimisation & .00 & & -.05 & \\
\hline Emotion dysregulation & -.03 & & .09 & \\
\hline Indirect home $\mathrm{x}$ dysregulation & .03 & & $.16^{* *}$ & \\
\hline Indirect school $\mathrm{x}$ dysregulation & .09 & & .02 & \\
\hline Indirect community $\mathrm{x}$ dysregulation & -.11 & & -.05 & \\
\hline Direct community $\mathrm{x}$ dysregulation & .11 & & $.15^{*}$ & \\
\hline Indirect political $\mathrm{x}$ dysregulation & -.02 & & -.10 & \\
\hline
\end{tabular}


Further, simple slope analyses were conducted to examine these significant interactions (see Figure 1). For the interaction between indirect home victimisation and emotion dysregulation, the slopes of the regression line between home victimisation and health risk behaviours were significant for both high emotion dysregulation $(\mathrm{B}=.28, p<.001)$ and low emotion dysregulation $(\mathrm{B}=-.28, p<.01)$. This suggests that girls with high emotion dysregulation, i.e., not able to regulate sadness and anger, performed more health risk behaviours when they had high exposure to indirect home victimisation compared to those who had low exposure to indirect home victimisation. Conversely, girls with low emotion dysregulation, i.e., better at regulating sadness and anger, performed less health risk behaviours when they had high exposure to indirect home victimisation compared to those who had low exposure to indirect home victimisation. For the interaction between direct community victimisation and emotion dysregulation, the slope was only significant for girls with high emotion dysregulation $(\mathrm{B}=$ $.39, p<.001)$. Girls with high emotion dysregulation performed more health risk behaviours when they had higher exposure to direct community victimisation compared to those had lower exposure to direct community victimisation. Among adolescent girls with lower emotion dysregulation, the association between direct community victimisation and health risk behaviours was not significant $(\mathrm{B}=-.06, p>.05)$.
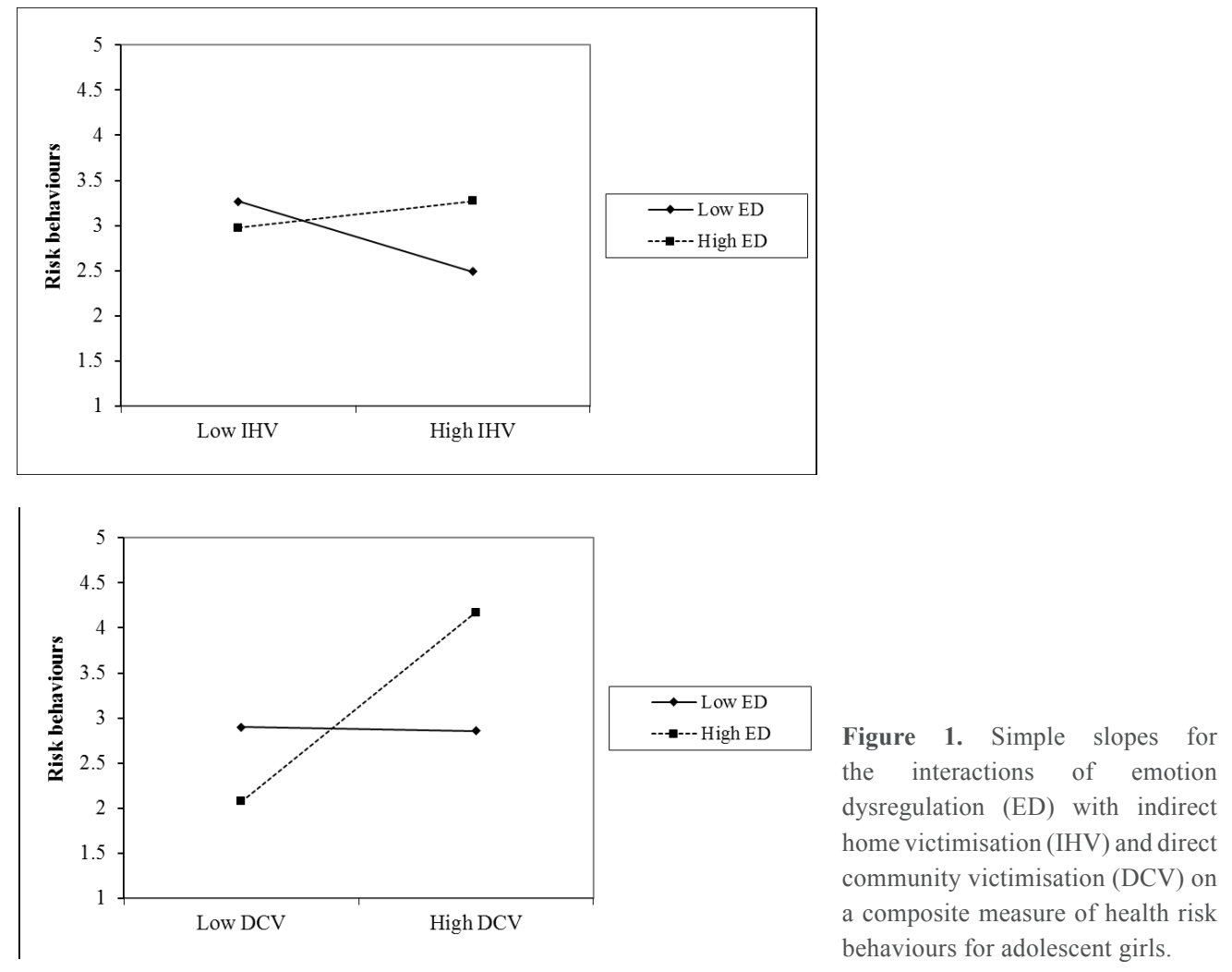

\section{Discussion}

This study builds upon previous literature by supporting the detrimental effects of exposure to violence across different contexts on health risk behaviours in South African adolescents, while providing additional information on the moderating role of emotion dysregulation and the patterns of gender. Consistent with other research (Begle et al., 2011; Lee, 2012; Moreira et al., 2008; Voisin et al., 2011), this current study showed that three out of five types of victimisation (indirect school victimisation and both indirect and direct community victimisation) were associated with increased risk for health risk behaviours among South African adolescents. Researchers (Harrison, Fulkerson, \& Beebe, 1997; Hessler \& Katz, 2010) have viewed the relationship between violence exposure and health risk behaviours in light of coping theory, suggesting that the engagement in risk behaviours might be one way individuals attempt to diminish negative affect (e.g., fear, nervousness) in the face of a stressful encounter (Cramer, 2000; Lazarus, 1993). Although it may result in a short-term 'soothing' effect (Tice, Bratslavsky, \& Baumeister, 2001), ultimately this coping method is non-adaptive: a study showed that among maltreated adolescents, drinking alcohol with a motive to cope with negative affect is predictive of other problems associated with alcohol use, such as getting into fights and neglect life responsibilities (Goldstein, VilhenaChurchill, Stewart, \& Wekerle, 2012).

Our results revealed that boys and girls differed in the associations between the types of violence victimisation and health risk behaviours. Specifically, after accounting for other types of victimisation, being a direct victim of community violence predicted health risk behaviours in boys, whereas exposure to indirect school victimisation predicted health risk behaviours in girls. This result is in line with studies by Begle et al. (2011) and Moreira et al. (2008), who found that exposure to community violence has an effect on boys' alcohol and drug use. Additionally, our $t$-tests showed that boys had more exposure to direct community victimisation than girls. In South Africa, boys may be more likely than girls to directly encounter community violence due to the fact that boys spend more time in the neighbourhood (Kaminer et al., 2013), which also potentially expose them to the highly available alcohol and drugs in many South African communities (Burton \& Leoschut, 2013), thus increases the likelihood of their health risk behaviours.

Although boys in our study also reported higher rates of indirect school victimisation than girls, this type of violence only had a negative influence on health risk behaviours in girls. The 2012 national school violence survey in South Africa showed that girls experience higher rates of sexual assault in school than their male counterparts, and are more likely than boys to report feelings of fear and anxiety associated with travelling to and from school (Burton \& Leoschut, 2013), suggesting that school violence generates high psychological 
stress for girls. In addition, an extensive literature has documented that girls are more prone to experiencing internalising symptoms than boys during adolescence (Hilt, Cha, \& NolenHoeksema, 2008; Mrug et al., 2008; Slone \& Mayer, 2015; Sui et al., 2018). Taken together, our results suggest that engaging in health risk behaviours could be one way of girls' attempt to cope with the adverse emotions aroused by exposure to school violence. This coping mechanism has been noted in Harrison et al. (1997), in which they found that adolescent girls exposed to physical and sexual abuse have an increased likelihood of alcohol and drug use, and a higher proportion of girls than boys use these substances to cope with painful emotions and to escape problems. Additionally, our study showed that health risk behaviours in girls emerged when school violence was indirectly encountered through witnessing, suggesting that girls may be susceptible to the negative influence of violence even if the level of exposure is relatively less intense than direct victimisation. Since the current study employed a cross-sectional design, future research should determine whether the gender differences in the effects of violence found in the current research are indeed resulted from these underlying processes.

In contrast to other studies (Brown et al., 2012; Hessler \& Katz, 2010; Wills et al., 2016), the current study showed that the inability to regulate sadness and anger did not directly influence health risk behaviours, possibly due to the reason that health risk behaviours in adolescents are influenced by a complex system of factors, such as mental health problems, attachment style, economic disadvantage, unhealthy family functioning, parental substance abuse, and peer influence (Feeney, Peterson, Gallois, \& Terry, 2000; Shek \& Liang, 2015; Spijkerman, Van den Eijnden, Overbeek, \& Engels, 2007; Pumariega, Burakgazi, Unlu, Prajapati, \& Dalkilic, 2014). An important finding in our study is that emotion dysregulation moderated the relationship between violence exposure and health risk behaviours in girls. Consistent with Bender et al. (2012), our $t$-tests showed that girls had higher emotion dysregulation than boys. Thus, for girls, their inability to regulate negative emotions may be a risk factor that exacerbates the consequences of violence exposure. Specifically, the current study showed that the effects of indirect home victimisation and direct community victimisation were associated with more health risk behaviours in girls when they were unable to regulate sadness and anger. This result is consistent with research by Kliewer et al. (2017), who demonstrated positive associations between cumulative psychosocial risks (including violence) and internalising and externalising symptoms in South African adolescents who had high emotion dysregulation. Similarly, in a sample of young adult women experiencing posttraumatic stress symptoms, emotion dysregulation increased the risk for later alcohol and drug use (Tull, Bardeen, DiLillo, Messman-Moore, \& Gratz, 2015). From a developmental perspective, adolescent girls may be more vulnerable to psychological distress than boys because girls process stressors differently, such as having a tendency to ruminate - a repetitive and recurrent thinking about the traumatic event and its consequences (Dunn, Gilman, Willett, Slopen, \& Molnar, 2012). This may create heightened negative affect for girls that interfere with their emotion regulation capacities to cope with violence effectively.

\section{Implications for Interventions}

The results of this study provide avenues for the development of interventions to reduce health risk behaviours in adolescents exposed to violence. Schools can be prime sites for interventions to reach a large number of adolescents and combat the negative influence of violence on their development (Gevers \& Flischer, 2012). For example, an evidencebased 'Life Skills Training programme' has been developed and implemented in the USA to combat health risk behaviours in youth through skills training in reducing stress and negative emotions, as well as resisting peer and media pressure to smoke or use substance. The programme has shown to not only positively influence the targeted behaviour (e.g., drug use) but to also generalise to other behaviours (e.g., HIV risk behaviour) (Griffin, Botvin, \& Nichols, 2006). Other school-based interventions that use similar approaches to enhance social skills and self-management skills have also been found effective in reducing marijuana and alcohol use in adolescents (Das, Salam, Arshad, Finkelstein, \& Bhutta, 2016; Scott-Sheldon, Carey, Elliott, Garey, \& Carey, 2014). Akin to these interventions, a programme for South African adolescents specifically focused on health education, skills training in reducing stress and negative emotions, and resistance to peer pressure in highrisk situations may prevent health risk behaviours.

As informed by our result on the moderating role of emotion dysregulation in the association between violence exposure and health risk behaviours in girls, gender-specific risk behaviour interventions may be especially helpful. Emotion regulation reflects a fundamental aspect of youth development and is a modifiable skill that can be trained and acquired (Compas et al., 2014; Kliewer, 2016). Thus, for girls, one key approach in skills training in interventions could be strengthening their emotion regulation capacities to enhance adaptive coping and help them modulate their behaviours in high risk situations, such as violence exposure. Indeed, research has found that emotion regulation training is effective in combating risk behaviours - for example, a programme that consisted of 12 after-school sessions that focused on emotion education and building skills in emotion regulation (e.g., recognising feelings in self and others), successfully reduced early sexual activities even after one year follow up among adolescents (Houck et al., 2016). In addition, it may also be beneficial to involve parents/caregivers in such a programme to provide them with the information about the associations between violence exposure, emotion dysregulation, and health risk behaviours in adolescents; but more importantly, to enhance their own emotion regulation capacities, for them to learn how to provide adequate emotional support to their children, and improve child-caregiver relationship and parenting style. These positive influences in the family 
context are important for young people to appraise their feelings, learn about strategies for emotion management, and achieve competence in controlling emotions, thereby buffer the negative effects of violence exposure (Kliewer et al. 2004; Thompson \& Meyer, 2007).

\section{Limitations and Directions for Future Research}

The results of our study offer novel insights, however, several limitations should be noted. This study is cross-sectional in nature, thus, causal relationships cannot be inferred and the results should be interpreted with caution. Although longitudinal studies have established the negative impact of violence on health risk behaviours (Begle et al., 2011; Wilson, Woods, Emerson, \& Donenberg, 2012), a bidirectional relationship is possible, such that engaging in risk behaviours may predispose adolescents to more violence exposure, for example by their involvement in drug dealings (Duke, Smith, Oberleitner, Westphal, \& McKee, 2018; van der Merwe \& Dawes, 2007; Mrug \& Windle, 2009). To establish the causal relationships, more longitudinal evidence is needed to fully understand the association between exposure to violence and health risk behaviours in adolescents. Another limitation is that due to the differences in scale formats and the small sample sizes for the participants regarding each risk behaviour, the risk behaviour variable in our study was computed as a composite score - consisting of smoking, alcohol use, soft and hard drug use, and risky sexual behaviour. Although the results are able to provide an overview of the effects of violence on adolescent health risk behaviours, information on the effects of violence on specific behaviours is lost, prohibiting our ability to draw conclusions about the effect of exposure to violence on, for example, alcohol use versus soft drug use. Future research should distinguish health risk behaviours in order to provide refined and targeted recommendations based on the effects of violence on separate behaviours. Unexpectedly, we also found that girls with low emotion dysregulation reported less health risk behaviours at high levels of exposure to indirect home victimisation, compared to low levels of exposure. This may suggest that low emotion dysregulation is a protective factor for risk behaviours only when the frequency of violence exposure reaches a certain threshold. Future research may explore this in more details. Lastly, only victimisation in the community context was differentiated into indirect and direct exposure in our study. Future research may examine both indirect and direct forms of victimisation for all contexts of exposure, as indirect and direct victimisation across contexts have been found to have a differential impact on youth psychological and behavioural adjustment (Barbarin, Richter, \& de Wet, 2001; Shields et al., 2009; Vermeiren, Schwab-Stone, Deboutte, Leckman, \& Ruchkin, 2003). This differentiation of violence could potentially enhance the understanding of the negative effects of different types of victimisation and provide more refined recommendations to alleviate these effects.

\section{Conclusion}

This study provides insight into the associations between violence exposure across multiple contexts, emotion dysregulation, and health risk behaviours in South African adolescents in the Western Cape Province. Gender patterns were found in the association between violence exposure and health risk behaviours and that emotion regulation moderated the effects of violence for girls. The results of the study have implications for the development of interventions to reduce health risk behaviours in adolescents exposed to violence. Building competencies in emotion regulation in adolescents, particularly girls, may be helpful to strengthen resilience to the negative effects of violence on health risk behaviours.

\section{Footnotes}

${ }^{1}$ A former system of institutionalised racial segregation in South Africa (1948 - 1994) in which access to economic resources, medical care, and educational and employment opportunity were restricted for people socially classified as "non-white". 


\section{Chapter 6}

Developmental Assets in South African Adolescents Exposed to Violence: A Qualitative Study on Resilience 


\section{Abstract}

Violence exposure is associated with psychological and behavioural maladjustment in adolescents. Yet, not all adolescents exposed to violence experience negative symptoms. Resilience is an outcome that is in part determined by multiple protective factors, or developmental assets, that protect adolescents from the negative influence of encountered stressors and allow them to attain positive developmental outcomes. A qualitative study was conducted to acquire an in-depth understanding of the developmental assets across different layers in the ecological system that promote positive psychological and behavioural functioning in South African adolescents exposed to violence. Semi-structured individual interviews were conducted with a multi-ethnic group (black, white, and people of mixed heritage) of South African adolescents (boy: $n=17$; girl: $n=13$; age: $14-19$ years) from seven schools in Cape Town. Adolescents reported both internal and external assets that helped them adaptively cope with violence exposure. The internal assets identified in the study entail individual characteristics and skills, including commitment to learning, positive values, positive identity, social competencies, and emotional insight. The external assets were boundaries and expectations, social support from adolescents' peers, family, school, and community, and adolescents' constructive use of time. The findings of the study may inform strengths-based interventions to enhance internal and external assets in adolescents at risk for violence exposure and promote positive developmental outcomes. Interventions may focus on building adaptive coping skills in adolescents to overcome the adversity and involve key stakeholders from major developmental domains to optimise supervision and social support.
Violence and crime rates are exceptionally high in South Africa. Recent statistics show that on average 58 people are murdered daily, and violence against women and children remains a serious public health concern (Statistics South Africa, 2019). Studies have found that violence exposure among South African adolescents is prevalent, and they can encounter violence directly (personal victimisation) and indirectly (witnessing or hearing about violence) across major developmental domains such as home, school, and community (Kaminer, du Plessis, Hardy, \& Benjamin, 2013; Shields, Nadasen, \& Pierce, 2009; Sui et al., 2018).

Violence is inherently stressful and may produce heightened and persistent emotional arousal, hypervigilance, and concentration problems (Mrug, Loosier, \& Windle, 2008; Sui et al., 2019). These emotional and cognitive difficulties may further interfere with healthy functioning in other adaptation domains such as self-regulation - the ability to control one's attention, emotions, and behaviours (Masten \& Coatsworth, 1998). As a result of this disrupted process of normative stress adaptation, adolescents can experience difficulties in utilising their strengths and resources to cope successfully with violence exposure, and subsequently experience psychological and behaviour problems (Heinze, Stoddard, Aiyer, Eisman, \& Zimmerman, 2017; Mrug et al., 2008). For example, violence can interfere with the capacity to regulate emotions of sadness and anger (Kliewer et al., 2004, 2017, Sui et al., 2019). It can also dampen one's goals and aspirations, and elicit feelings of hopelessness through the perception that there are few pathways for recovery and progress in life (Bonanno, 2007). In response to violence, adolescents may also engage in alcohol and drug use in an effort to cope with negative emotions, block out their feelings, and escape problems (Harrison, Fulkerson, \& Beebe, 1997; Hessler, 2008). Furthermore, aggression and violent behaviours have been found in adolescents who had prior exposure to violence, partially through a process of emotional desensitisation and social learning that violence is a sanctioned way of handling conflict, which in turn reinforces externalising symptoms (Choe, Zimmerman, \& Devnarain, 2012; Mrug, Madan, \& Windle, 2016; Voisin $\&$ Hong, 2012). In addition, violence exposure may have deleterious effects on adolescents' psychological functioning and behaviours when multiple exposures are experienced (i.e., poly-victimisation; Mrug et al., 2008; Sui et al., 2018). This in part could be due to the disruption of violence in multiple interpersonal domains that depletes one's adaptation resources (Finkelhor, Ormrod, \& Turner, 2007).

However, not all young people exposed to violence experience negative developmental outcomes, as some grow up as individuals who are positively adjusted (Ward, Martin, Theron, \& Distiller, 2007; Ostaszewski \& Zimmerman, 2006). For example, a U.S. study found that $59 \%$ of adolescents showed healthy mental health functioning seven years after exposure to community violence (Jain, Buka, Subramanian, \& Molnar, 2012). This suggests 
a variation in young people's vulnerability to the negative impact of violence exposure, as some may be more resilient than others.

Resilience is defined as the successful coping with or the overcoming of risk and adversity, and the development of competence in the face of severe stress and hardship (McKnight \& Loper, 2002). Over the past two decades, the understanding of resilience has transformed from a trait-oriented approach to a process-oriented approach (Masten, 2011). That is, instead of viewing resilience as an intrinsic and stable trait, the emerging approach emphasises resilience as a life outcome that is in part determined by multiple factors that protect adolescents from the negative influence of encountered stressors (Chmitorz et al., 2017). These protective factors contribute to a dynamic process that may account for individual differences in patterns of adaptation, function, or development that occur during or following the experiences of risks or adversity (Masten, 2011).

To foster resilience in adolescents exposed to violence, an emphasis has been put on identifying and building developmental assets to minimise the risk for maladjustment (Jain \& Cohen, 2013). The developmental assets include internal assets (e.g., personality traits, prosocial skills, attitudes and beliefs) and external resources from major developmental domains (e.g., support from home, school, and community) that a young person needs to buffer the negative effects of violence and attain positive developmental outcomes (Scales, Benson, Roehlkepartain, Semsa, \& Van Dulmen, 2006; Jain et al., 2012). Both internal and external protective factors may promote resilience in young people through the mechanism of utilising their assets and resources from different areas to achieve positive adaptation to the adversity (Harvey, 2007).

For example, a longitudinal study found that prosocial skills such as cooperation, assertion, responsibility, and self-control have long enduring effects to reduce aggression among physically abused children (Holmes, Yoon, Voith, Kobulsky, \& Steigerwald, 2015). Prosocial skills allow the child to become adept at reading social norms, facial expressions, intentions, empathy, and having realistic expectations of social situations. These skills contribute to adaptation in high risk situations, which in turn result in the child resorting to problemsolving rather than aggression (Holmes et al., 2015). Other skills such as the ability to regulate emotions of sadness and anger, and adaptive coping (e.g., problem solving) are associated with better psychological outcomes in adolescents exposed to violence in the community (Aitcheson, Abu-Bader, Howell, Khalil, \& Elbedour, 2016; Kliewer et al., 2004).

In addition to internal protective factors, supportive relationships spanning across different developmental domains in a young person's ecology may also serve as influential factors for their adaptation to violence. For example, one longitudinal study found that external resources such as support from peers and family are strong predictors for emotion resilience across time in adolescent witnesses and victims of violence (Jain et al., 2012). These social support structures have also been found effective in reducing violent behaviours in adolescents exposed to school violence (Duggins, Kuperminc, Henrich, Smalls-Glover, \& Perilla, 2016) and childhood maltreatment (Pérez-González, Pereda, Guilera, \& Finkelhor, 2017)

Traditionally, much of the research on adolescent exposure to violence measures a "deficit model" that focuses on the negative consequences of violence and the associated risk factors (Morgan \& Ziglio, 2007). However, while the deficit model is important and necessary to identify levels of needs and priorities, we feel it needs to be complemented by other perspectives. Relatively few studies sought to provide a holistic understanding of the protective factors that help youth cope adaptively following violence exposure (Jain \& Cohen, 2013; Ward et al., 2007; Herrenkohl, Aisenberg, Williams, \& Jenson, 2011). This research is lacking particularly in South Africa, despite the country's high prevalence, persistence, and magnitude of violence exposure. As such, there is a need to enhance the understanding of strengths and protective processes that promote competence and positive development in adolescents.

The current study was designed to fill part of the gap in the literature by conducting qualitative research with the aim to explore the dynamic process of resilience in Sout African adolescents. Few studies on understanding resilience in adolescents are qualitative in design, resulting in a dearth of in-depth information, particularly in the context of violence exposure. A qualitative study will allow us to obtain an in-depth understanding of an array of developmental assets that can enhance resilience in adolescents exposed to violence and capture the nuances of these protective factors in different adolescents. Since resilience is modifiable (as opposed to a stable trait), such an in-depth understanding may help inform the development of strengths-based interventions that accentuate positive capability and create opportunities for young people to adapt in an environment where violence is highly prevalent.

\section{Methods}

\section{Design}

Semi-structured interviews were conducted with adolescents to gain an in-depth understanding of an array of factors at the individual, peer, family, school, and community levels that may buffer the adverse effects of violence exposure. 
Due to the sensitive nature of violence, data were collected by means of individual interviews to ensure comfort and to protect the privacy of the participants. Details of violence exposure were not asked in the interviews to minimise the risk of re-traumatising the participants. During the interviews, participants were invited to discuss their thoughts and behaviours after violence exposure; what they would find helpful for young people to cope with violence, as well as the activities, things, or people that they thought would and did help them cope following their own experiences of violence. A school counsellor was available for counselling service in case any participant reported or showed signs of distress by talking about the topic (no such referrals were made in the current sample).

\section{Participants}

Participants were purposefully recruited from seven public schools ${ }^{1}$ (two primary and five secondary schools) in Cape Town, South Africa. The study sample comprised 30 adolescents (boy: $\mathrm{n}=17$; girl: $\mathrm{n}=13$ ) between the age of 14 and 19 who identified themselves as black ( $\mathrm{n}=14)$, people of mixed heritage $(\mathrm{n}=15)$ and white $(\mathrm{n}=1)$. The participants resided in communities where physical and sexual assault, gang violence, and murder are highly prevalent. More specifically, Cape Town has the highest recorded rates for murder, robbery, and property-related crimes compared to other cities in South Africa (Urban Safety Reference Group, 2019). All adolescents in the interviews reported to have encountered violence either directly or indirectly (e.g., witnessing).

\section{Data Collection}

Recruitment of the participants in the local communities (instead of the schools) imposed challenges given the high prevalence of crime and the hard-to-reach circumstances of the adolescents at risk for violence exposure. As such, the school context was used as an entry point to contact participants, as it is one of the major developmental contexts where a large number of adolescents are gathered on a frequent basis. Information packages that contained details about the purpose and aims of the study were emailed or personally delivered to 15 public schools in Cape Town. Seven schools (47\%) responded positively about participation. Upon receiving permission from the school principals, the researcher (first author) and two trained female research assistants each went to different schools for recruitment of adolescents. Eligible participants included adolescents between the age of 14 and 19 who lived in violence-prone communities. Adolescents who agreed to participate did the interview at their free period (no lesson at the time of participation) or after school in an empty classroom available at the time. The interviews ranged between 40 to 60 minutes and were conducted in a language preferred by the participant (English, Afrikaans, or Xhosa). Twenty-six interviews were done in English, two were in Afrikaans, and two were in Xhosa. Each participant received snacks as incentives

\section{Data Analysis}

Theoretical thematic analysis by Braun and Clarke (2006) was conducted to understand the factors that enhance South African adolescents' resilience to the negative effects of violence. The 40 developmental assets framework (Search Institute, 2017) focuses on a range of positive influences and supports from families, schools, and communities (external assets) and the personal strengths, values, and commitments that are nurtured within young people (internal assets) to achieve healthy psychosocial development. This framework was used as a guideline to grasp the essential factors that positively influence resilience in the current sample of South African adolescents who had exposure to violence. New themes other than the ones listed on the framework (Search Institute, 2017) were allowed to emerge, given that they had theoretical support and empirical evidence in the literature. Data were analysed based on Braun and Clarke's (2006) six-phase method using the Atlas.ti software.

\section{Rigour}

To ensure the rigour of the study, Lincoln and Guba's (1985) principles of transferability, confirmability, credibility and dependability have been guiding principles. The interviewers each kept a diary to record any thoughts and reflection emerged during or after the interview for debriefing. Following each interview, the research team consulted with one other to ensure emerging themes were in line with the primary research aim, to include additional probes in the interviews if necessary, and to decide whether data saturation was reached. Upon completion of data collection, research assistants transcribed the interviews verbatim. Afrikaans and Xhosa interviews were translated into English. In addition, another researcher with similar academic experience was involved in the data coding process along with the primary author who each independently coded the interviews. Any discrepancies between the researchers were discussed and modified to reach agreement of all the themes.

\section{Ethics}

Approval to conduct the study in public schools in Cape Town has been granted by the Western Cape Department of Education (20180504-1777), along with the ethics approval from the Human Sciences Research Council in South Africa (REC 1/21/02/18). Consent from the participants over 18 years old as well as both parental/caregiver consent and assent from the participants under 18 years old were obtained.

\section{Findings}

The factors that positively influenced resilience in adolescents who had exposure to violence were grouped into external assets (Group 1) and internal assets (Group 2). Within these larger groups, themes were identified: External assets entail two themes, namely, boundaries and 
expectations, and social support; internal assets consist of six themes, which are commitment to learning, positive values, positive identity, social competencies, and emotional insight. The groups, themes, and sub-themes are presented in Table 1.

\section{Group 1: external assets}

\section{Theme 1a: boundaries and expectations}

Many adolescents followed rules and instructions set by their parents that aimed at preventing them from being exposed to violence. These parents advised their children to stay indoors, not engage in physical fights with peers, not go near violence-prone areas or walk alone late at night, and stay away from peers who are involved in gangsterism, smoking, and substance use. The adolescents said that these family rules were helpful because they believed they were still young and needed information and guidance from adults to protect themselves, especially when they lived in communities where crime and violence were prominent:

All that I am proud of is my parents that tell me not to play in the road, and to stay indoors... They say if you respect your parents you will go far in life. (male, 15 y.o.)

Table 1. Themes and sub-themes of developmental assets in South African adolescents

\begin{tabular}{|c|c|c|}
\hline Asset domain & Themes & Sub-themes \\
\hline \multirow{3}{*}{ External assets } & Boundaries and expectations & $\begin{array}{l}\text { Family rules } \\
\text { - Family monitoring } \\
\text { - School rules } \\
\text { - School monitoring } \\
\text { - School teaches values } \\
\text { - Positive social norm } \\
\end{array}$ \\
\hline & Social support & $\begin{array}{l}\text { - Family support } \\
\text { - Peer support } \\
\text { - School support } \\
\text { - Community support }\end{array}$ \\
\hline & Constructive use of time & $\begin{array}{l}\text { - Leisure activities } \\
\text { - Religion } \\
\end{array}$ \\
\hline \multirow{5}{*}{ Internal assets } & Commitment to learning & $\begin{array}{l}\text { Achievement motivation } \\
\text { - School engagement } \\
\end{array}$ \\
\hline & Positive values & $\begin{array}{l}\text { - Attitude of violence } \\
\text { - Restraint from substance use } \\
\text { - Empathy }\end{array}$ \\
\hline & Positive identity & $\begin{array}{l}\text { Positive view of self } \\
\text { - Personal power } \\
\end{array}$ \\
\hline & Social competencies & $\begin{array}{l}\text { - Seek social support } \\
\text { - Peaceful conflict resolution } \\
\text { - Resistance to potentially risky situations }\end{array}$ \\
\hline & Emotional insight & $\begin{array}{l}\text { Emotion regulation } \\
\text { - Positivity } \\
\text { - Reflective thinking }\end{array}$ \\
\hline
\end{tabular}

In addition to rules in the family, many adolescents reported that they had parents who were attentive and monitored their daily activities. For example, those parents checked up on the adolescents throughout the day to find out their whereabouts, and made sure they were safe and with the right people. Parental monitoring was seen positively by the adolescents as they felt that their parents were involved in their lives, which made them feel protected and appreciated:

They would check on me just to make sure I didn't go into a wrong path. I feel it's very supportive because I know that my parents do actually care. (male, 14 y.o.)

However, some parents were disinterested and uninvolved in the adolescents' lives. Many adolescents also lived in single parent households due to parents' divorce or death. The lack of or inadequate supervision and monitoring of the parents may lead to risk behaviours in adolescents as their way to seek attention and approval from others:

Because some people don't get attention at home and then they come to school and they are violent to look for attention from the teachers. (male, 15 y.o.)

Moreover, the schools that the adolescents went to had rules they needed to follow and there were consequences if they engaged in violence, including threats, or engaging in verbal or physical violence towards others. Warnings and detentions were common as punishment for violent behaviours to create a school climate that communicates that violence is not tolerated. Many schools also had surveillance systems such as cameras and security guards who patrolled around the school to ensure the safety of the adolescents:

The teachers said once you fight in school, you get direct detention without any warnings... You go and write the code of conduct and finish it in a specific time. It helps because as you are writing you are also reading the school rules. (male, 14 y.o.)

To prevent violence, many schools incorporated moral values such as respect, kindness, peace, and harmony, and included topics of anti-violence in their education curriculum. These values and topics were taught in Life Orientation lessons and through plays and shows in school. Occasionally the schools invited guest speakers whom the adolescents could identify with, such as a young man who showed high aspirations and talents in rap music and encouraged the adolescents to be kind to one another and engage in activities that help them achieve their goals in life. As such, the schools helped adolescents understan that violence is wrong and that they should instead engage in prosocial behaviours: 
They [teachers] say you shouldn't bully other people, that you must treat your peer as you love yourself, sometimes [demonstrated] in plays. It is helpful because we should all help each other and help people in need. (male, 14 y.o.)

In addition, social norms in the adolescents' environment can also influence their development. For example, family role modelling was evident in several adolescents' narratives as they described that their parents were calm and never approached problems or conflicts with violence, nor did they engage in violence-related behaviours such as alcohol- and drug use. Growing up in a family with positive influence seemed to motivate the adolescents to keep away from violence and its associated behaviours such as substance use, as they did not want to be the first in their family to conduct these behaviours:

My father never laid a finger on my mother and they never fought... It is my goal to follow their footsteps. I'm still achieving that and I will make sure it will happen. (male, 14 y.o.)

Similarly, some adolescents reported positive peer influence, such as having a friend that worked hard to achieve goals, a friend who set a good example by resolving a violent encounter in a non-violent way, or a friend who encouraged them to stay away from violence-related behaviours, such as smoking and drinking. These adolescents believed it is important to associating themselves with peers of positive influence to help them stay motivated and go further in life:

I find him [friend] an inspiration upon me and we do many things together like we hike, do stuff that's going to help us or benefit ourselves, not sit around the corners and do drugs and smoke. (male, 16 y.o.)

\section{Theme 1b: social support}

Some adolescents reported that their parents showed support by being there for them after violence exposure. They were encouraged to communicate openly with their parents about the details of the event. Some adolescents said their parents were proactive to help them resolve the problem of violence exposure, such as talking to the teacher or the perpetrator's parents. Family support has brought adolescents comfort and made them feel assured that they could rely on the family:

My mom just sits with me and then we have a nice conversation and she talks to me like she's my best friend... She makes me feel like I'm comfortable with her, can speak with her about anything. (female, 14 y.o.)
Conversely, many adolescents did not feel comfortable opening up to their parents after violence exposure because, they said, their parents were judgmental and often blamed them for the situation. The adolescents described their frustrations towards their parents: for not making an effort to understand them or not taking what they say seriously. For example, one female adolescent was sexually assaulted repeatedly by a family member. When she finally had the courage to tell her family about the incidents, they refused to believe her, and this has left her feeling helpless and unable to trust anyone:

My father didn't believe me. So in a way my family brushed it off and nothing happened to it. I felt useless. I felt like everybody can take something away from me. I can't open up, it left me broken. (female, 17 y.o.)

From the interviews, it became apparent that peer support is important in the adolescents' lives. Friends can make them happy and being around friends can take their mind off the negative experience of violence. When some respondents felt sad after being bullied in school, their friends showed support such as hugging and giving words of encouragement, buying snacks for the victimised friend, or visiting them and keeping them company. Many adolescents also stated that it is more helpful to talk to a person of similar age rather than someone older because young people often share similar experiences of violence, and they are more able to show understanding and provide comfort for one another:

My cousin has been through a lot of things as well. I know that we are there for each other. She has been through bullying. We have been through similar things so we know if I need help then I can talk about it. (male, 14 y.o.)

In contrast to the reports about bullying in school, some adolescents reported to enjoy the school environment and described the school as "a family". They enjoyed being with peers and they believed the school was the safest place they could be, compared to the areas they lived in. They described that some of their teachers and principal in schools were caring for the learners who had been victimised and treated them as their own children. They responded immediately when there was violence in school and approached the incident fairly by listening to both sides of the story from the victim and the perpetrator. They treated the learners with sensitivity and tried to understand the full story first before deciding on any discipline. For example, one adolescent witnessed her father's death from gang violence, and started to have anger problems and became aggressive towards peers. She said that in reaction to her behaviour - rather than disciplining her - the school showed understanding to her aggression and approached her with sensitivity, because they understood her behaviours may be due to the violence exposure, and that it was more important to meet her current emotional needs: 
Mr. T, he never just does anything like calling my mother for the things that I do. He just sits me down and speaks to me like a parent... Ms. K, she knows that even my teacher goes to her complaining, she just speaks to me and asks me to change my attitude, and I actually does it, because at the end of the day, they are helping me to be where I want to be. They are not judgmental at all. (female, 17 y.o.)

However, many adolescents said some of their teachers should pay more attention to them because they sometimes neglected the incidents of violence, or did not attempt to understand the full story of violence. According to the respondents, this has led some teachers to jump to conclusions and to punish the learners without attending to their emotional needs. These teachers set academics as priority and often blamed the learners for not engaging in school work and neglected the fact that these young people had been traumatised by violence exposure. The adolescents wanted the teachers to show more interest, understand them on a personal level, and treat everyone fairly instead of only caring for the learners they like:

There have been nights where I don't sleep, where I've been crying myself to sleep. There's nights where I can 't do anything because I can't focus, I am focused on whatever happened at school that day. Not a lot of teachers understand that and they think it's because you're lazy or you don't take your school priorities seriously. (female, 14 y.o.)

Some schools provided counselling support to learners who experienced psychological or behavioural difficulties after violence exposure. Adolescents felt supported after using this service and they believed it provided a safe place where they could speak to someone when they did not feel comfortable to speak to other adults such as teachers. Furthermore, the counselling service in school has elevated some of the stress off the teachers, who are often too busy to respond to the incidents of violence in school due to heavy teaching obligations:

Ifeel safe with telling them [school counsellors] my experiences. They have done a lot of stuff for learners that are not doing well. They have stuff to keep you busy with, there's a swing, there are toys and stuff. They are kind. Sometimes teachers are busy and have to see to the class and can't pay full attention to you, so they help. (female, 14 y.o.)

When asked about possibilities of support in the communities, the adolescents suggested that due to the high rates of violence in their communities, they felt safe when their neighbours paid attention to whether there were criminal activities happening around one other's houses, and when there was a "neighbourhood watch" (volunteers who patrol the community). Since many adolescents were disappointed in the police's slow response to crimes in the communities, they believed that living in a community where people are connected and caring is an assuring experience and creates a cohesive environment:
I find that sometimes the community takes things into their own hands. Like, if they find a burglar then they themselves try to take care of it. And that in turn will make sure that other feels a bit safer knowing that there are the neighbours protecting you and that the neighbours are watching out for you... So instead of the police that is there only when they are called, the neighbours are always there. (female, 15 y.o.)

\section{Theme 1c: constructive use of time}

Adolescents reported that they engaged in hobbies and leisure activities to cope with violence. These included playing sport, dancing, reading, taking a walk, and listening to music. They enjoyed these activities because they could do what they were good at, which brought them a sense of accomplishment and because these activities helped them take their mind off the victimisation experience. Engaging in positive hobbies and leisure activities also protected them from high-risk situations where they could be potentially exposed to violence and interact with peers of negative influence:

Rugby makes me feel so much different. It keeps me out of trouble and keep me away from the wrong friends, from the wrong things like violence. (male, 14 y.o.)

Furthermore, religious beliefs and practices were identified as another major strategy that adolescents used to cope with violence. Adolescents sought strength in their spiritual beliefs to help them go through difficult times. They relied on religion as an important support system where they could gain a sense of purpose and feel motivated in life:

How I cope with violence is I read the bible, I read testimonies of people, like, this is how I used to be and this is how I am now, so it kind of helps me, it gives me more confidence, it helps me believe in myself and I want to make a change. (female, 15 y.o.)

\section{Group 2: internal assets}

\section{Theme 2a: commitment to learning}

Many adolescents believed that focusing on their school work is particularly important, as education paves an avenue for a better life and is something they must currently focus on to make a difference in their future. Moreover, adolescents reported that they had dreams and plans for their futures, wanting to become a "pilot", "firefighter", "doctor", "engineer", and "rugby player". They were determined to study hard and achieve these goals because they wanted to make their family proud, support their family financially, and help the family move out of violence-prone areas when they become successful:

They [adolescents] should basically mostly focus on their school because if they did a good job they can move out of that bad environment, they can move to a better 
environment, feel relaxed because they know that they have achieved something that probably their parents could not achieve. (female, 14 y.o.)

The adolescents mentioned that such dreams and aspirations helped them stay focused in life, forget about the violence they encountered, and dissociate themselves from violence and violence-related behaviours. For example, when the interviewer asked the adolescents about their opinions on using alcohol or drugs to cope with violence, one said:

I don't want that lifestyle. I have goals to achieve and I am not going to let that get in my way. (male, 16 y.o.)

\section{Theme $2 b$ : positive values}

Several value systems that helped them stay away from violence and unhealthy behaviours associated with violence exposure were identified. Many reported an attitude against the use of violence, such as "I don't believe in violence" and "What it does to a person is wrong". Furthermore, when the interviewer asked about adolescents' opinions on alcohol and drug use in victims of violence, they believed that it is an ineffective way to cope with violence because of the negative consequences on health and it will lead them to follow the wrong paths in life:

I believe that maybe drinking and smoking and occupying yourself with those kind of activities keeps you away from your real purpose... It might take away the pain temporarily, but it will affect your whole life. (female, 15 y.o.)

Moreover, some adolescents were bullied by their peers, but instead of taking revenge, they demonstrated empathy and understanding of the perpetrator's situation and needs. They felt that the perpetrators are sometimes also victims of violence themselves, who can experience negative emotions and in turn take it out on others through the use of violence. Empathy has led to forgiveness and a few adolescents who were bullied eventually established friendship with the perpetrator:

We're actually friends now. I forgave him. We just spoke about why he'd done it. I understand because of the condition he was in. Because some people get emotional really quick and it can affect them... If I wouldn't forgive him, I would think about it all the time and I can also do something bad to him and I don't want to do it. (male, 14 y.o.)

\section{Theme 2c: positive identity}

Some adolescents reported a negative perception of themselves after being personally victimised by violence. They experienced internalising symptoms, such as feeling shameful and worthless:
Sad like I don't need to be in this life because the bully didn't appreciate me, so I felt like I didn't deserve to be in this life. (male, 15 y.o.)

On the other hand, some adolescents had a positive sense of self and they were happy and proud of the way they are. They described themselves as confident, kind, diligent, and worthy of love. These personal beliefs brought assurance to these adolescents and they did not let violence affect their self-perception in any negative way:

I am a kind, loving person. When I was going through verbal bullying, I just told myself I don't care what that person might think of me. There is still a million other people that still love and care about me. It does not really matter. (female, 14 y.o.)

Having a positive identity helped adolescents cope with violence exposure, as they were sure of themselves and their ability to overcome challenges and move on from the negative experience. They expressed the belief that they have control over what they can do about adversities. Instead of ruminating about the past, they let the negative experience pass and focused on living the current life:

I'd just tell myself, um, this is just another obstacle in your way so you're going to have to jump over it, just get over it. (female, 14 y.o.)

\section{Theme 2d: social competencies}

Some adolescents demonstrated social competencies by knowing when and how to look for adult support when they encounter violence. They went to the adults they trust, such as family members and teachers/principals. They believed these adults have experience in dealing with incidents of violence, can give advice to the adolescents who are affected by violence, and guide them to find solutions. Additionally, social competencies were evident in adolescents' peaceful conflict resolution in the instances where they encountered violence: Many chose to walk away from violence because they did not want to elicit more aggression between themselves and the perpetrator. Some chose to stand up for themselves in a non-violent manner and tell the perpetrators what they did is wrong. Some adolescents mentioned that when they would see friends that are aggressive towards one another, they would (try to) stop the fight and talk to both of them to resolve the conflict peacefully. The adolescents also emphasised the value of verbal communication to resolve conflicts, because it gives them an opportunity to find out if it is a misunderstanding, and to understand each other's thoughts and feelings instead of using violence:

I will try to make peace between the two people that are fighting, let them talk about the problem so that they will probably become friends. Communication is very important, 
because if there is no communication, you won't know how the person would feel. (female, 14 y.o.)

Social competency was also demonstrated in adolescents' ability to resist potentially risky situations. For example, some adolescents were aware that a few peers were in gangs. They also felt that many young people engage in smoking, substance use, and behave violently because they have a distorted belief that these behaviours are "cool" and make them manly. Although these peers of bad influence were present in their lives, the adolescents were able to identify the situations that put them at risk for maladaptive behaviours and thus refrained from behaving like or socialising with these peers:

I just don't want to be close to them because of the things they [young people] do. They smoke, drink at a young age already. They fight and they want to talk like gangsters...I think its stupid actually, because you can talk it out, it's not necessary to lift up your hand, it makes you weak inside. It shows that you are lacking something inside, there are problems. (male, 16 y.o.)

\section{Theme 2e: emotional insight}

The adolescents mentioned that there were incidents of violence exposure such as being verbally attacked or physically threatened by their peers in school and that such experiences triggered negative emotions. Although feelings of sadness and anger were commonly reported in the interviews, many were able to regulate these emotions. Several techniques and strategies were reported - some preferred going to a quiet place alone to help them stay calm. Others sought relief through meditation or the use of a stress ball.

Moreover, some adolescents encountered violence almost on a daily basis, yet they were able to regulate how they see the adversity by focusing on positivity. They believed being positive is a choice and since they already lived in a violence-prone community - something that cannot be changed immediately - they tried to see the situation from a different perspective so the experience of violence became more manageable:

If it's a serious moment or I am feeling sad, I am going to make a joke or find something to make me happy because I can't be sad. I can't deal with sadness so I choose to be happy. (female, 18 y.o.)

In addition, some adolescents reflected on the situation of violence they experienced by thinking thoroughly about how to react, and the impact of their responses on others. This reflective thinking pattern helped them to analyse the situation first and examine various options to approach the situation appropriately without responding with impulsivity that could elicit more violence:
I need a bit of time to myself to process everything and to think how it happened. Then I think, what do I do next and how do I go forward with this, and how can I go about it to talk to someone, like my mom. (male, 14 y.o.)

Interestingly, writing or journaling was commonly reported by adolescents to control negative emotions as well as to reflect on the violence they encountered. They wrote about the violence they experienced and how they felt after the incident. They found that writing helped to relieve the feelings that were bottled up after violence exposure and helped them to find solutions. Through writing, adolescents became more assured of who they are and gained a sense of control over the situation they encountered:

I write about it sometimes. It's like I have that stress and all that anxiety and use it as a relief... It helps because once you write, it's like okay I'm coping with this, I understand what's going on, but I'm better than this, I can move past all of this. (female, 16 y.o.)

\section{Discussion}

The everyday life of many South African adolescents is characterised by exposure to violence in one or multiple areas of their lives, i.e. in their homes, school, or communities. Much is already known about the negative consequences of such violence exposure, but given that many adolescents grow up to become positively adjusted adults (Ward et al., 2007), it is important to identify the factors that promote healthy development. Therefore, this study explored the factors that positively influenced resilience in a group of South African adolescents who had direct or indirect violence exposure. The findings suggest that both personal protective factors (internal assets) and environmental protective factors (external assets) may help adolescents adaptively cope with violence exposure and achieve resilient developmental outcomes, such as better psychological functioning and lower risk for smoking, substance use and aggressive behaviours (Sui et al., 2018; 2019).

Adolescents in our study reported three major external assets, namely social support, boundaries and expectations, and constructive use of time. These assets were accessible by some adolescents through their social networks that span across peers, home, school, and community. Consistent with the literature, social support, including nurturing parent-child relationships (Vanderbilt-Adriance \& Shaw, 2008), support from peers (Jain et al., 2012), a positive and caring school climate (Duggins et al., 2016), and a sense of belonging in the community (Ellis et al., 2015) are associated with positive behavioural and psychological adjustment, such as higher emotional resilience and less aggressive behaviours in adolescents 
exposed to violence. When the needs of connectedness are met, adolescents may feel safe and secure with others across different developmental contexts, which provides a secure base from where they can build psychosocial competence and self-reliance and engage in developmentally healthy activities (Hogue, Liddle, Becker, \& Johnson-Leckrone, 2002). As a result, this may buffer the stressful experience of victimisation and help adolescents positively adjust and "bounce back" from the adverse experience (Duggins et al., 2016). As evident in the interviews, the availability, attentiveness, and understanding of supportive peers, family, and teachers created a safe and comfortable space for adolescents to cope with violence. This opens an opportunity for them to develop more adaptive thoughts and coping behaviours such as taking their mind off the negative situation, thinking that they are not alone, and being more open to speak to someone and look for support.

Moreover, consistent with the literature (e.g., Duggins et al., 2016; Kliewer et al., 2004), our study found that rules and boundaries that discourage violence and promote safety, as well as regular monitoring of adolescents' actions by the adults in the family and school may reduce violent behaviours and substance use, and that adolescents may feel protected and assured. This suggests that having attentive teachers and parents who monitor and set rules and boundaries can be protective for adolescents in high-risk environments. Adolescents may perceive that the adults in their lives are interested and concerned about them, and establish a sense of self-worth and higher levels of self-regulation, which are important factors that promote resilience (Copeland-Linder, Lambert, \& Lalongo, 2010). In addition, our study revealed that social norms that discourage substance use and violence, and which are communicated either explicitly or implicitly by family and peers, encourage higher achievement motivation and effective problem-solving in conflict resolution in adolescents. Indeed, such behaviours can be acquired through social learning via direct observation and socialisation processes (Bandura, 1977), and having others modelling adaptive behaviours may thus be particularly helpful to promote healthy functioning. Other studies have also found social norm to be an important protective factor that promotes healthy and adaptive behaviours in adolescents, such as a reduction in gender-based violence (Rogers, Rumley, \& Lovatt, 2019) and alcohol use (Gersh, Lee, \& McCarty, 2019).

Furthermore, many South African adolescents in our study reported victimisation experiences across different contexts, such as witnessing or experiencing violence at home, bullying by peers in school, and violent crime in the community. Studies have found that multiple exposures to violence, i.e., poly-victimisation, may have more deleterious effects on one's behaviours and psychological functioning (Mrug et al., 2008; Sui et al., 2018). This in part, could be due to the reason that more people and more environments in an adolescent's life are associated with reminders of violence, which may contribute to the depletion of external resources such as supportive interpersonal relationships across multiple settings, and thus interfere with normal coping (Finkelhor, Ormrod, \& Turner, 2007). While some adolescents may experience a state of hypervigilance that contributes to internalising and externalising maladjustment; others, however, achieve resilient outcomes as they are able to utilise their internal assets to positive adapt in the situation of violence exposure, despite that the other resource domains (e.g., social support) may be affected (Harvey, 2007).

The internal assets we found in this study included commitment to learning, positive values, positive identity, social competencies, and emotional insight. More specifically, the adolescents indicated that they have positive beliefs about themselves and have high aspirations to achieve in life. They were able to utilise adaptive coping skills and social skills to establish meaningful and supportive interpersonal relationships. They also reported that they had competencies to reflect on and regulate their negative emotions. These social and cognitive skills serve as buffers to the negative impact of violence exposure and have been reported in other studies (e.g., Aitcheson et al., 2017; Holmes et al., 2015; Kliewer et al., 2004; Sui et al., 2019). The internal assets reflect individuals' competencies, skills, and self-perceptions, which play an important role to help adolescents cope with stress and promote adaptive psychosocial functioning (Scales, Benson, Leffert, \& Blyth, 2000).

\section{Implications for Interventions}

Through the qualitative approach of this study, we found that the internal and external assets reported by the adolescents are dynamic and that they are related to the nature of violence exposure and one's developmental context. For example, adolescents have their own definitions of positive identity and have different ways of regulating their emotions, e.g. via reflective thinking or writing. Moreover, some may experience less social support than others as they were exposed to more violence across their major developmental contexts, thus less available and reliable social support from these major contexts. The varied profiles of developmental assets account for the individual differences in adolescents' adaptation following violence exposure, which in turn affect their developmental outcomes (Masten, 2011). Since resilience is not an inherent and static trait (Fergus \& Zimmerman, 2005), there is an opportunity for strengths-based approaches to facilitate and sustain positive development and resilience in adolescents at risk for violence exposure.

Several interventions have shown effectiveness in promoting positive internal and external adjustment by enhancing adolescents' skills and social resources. For example, The Resourceful Adolescent Programme (Shochet et al. 2001) was found effective in reducing depressive symptomatology and hopelessness in adolescents. It incorporates 11 group sessions in schools aimed at affirming adolescents' existing strengths and assets, and enhancing problem solving skills, self-management skills to cope with stress, and interpersonal skills to strengthen support networks (e.g., positive family relations). Similarly, the Life Skills 
Training (Griffin, Botvin, \& Nichols, 2006) is a school-based programme that provides 30 sessions to adolescents ranging from developing stress management and emotion regulation skills to the social skills necessary to resist peer and media pressure to engage in health risk behaviours. The programme has shown to reduce drug use and HIV risk behaviour.

While these interventions target internal assets by building psychosocial skills, the Multidimensional Family Prevention Programme (Hague, Liddle, Becker, \& Johnsonleckrone, 2002) focuses on forging durable external connections with others in one's social systems (e.g., family, school, prosocial peer groups, and recreational and religious institutions). This family-based programme has shown effectiveness in enhancing psychosocial wellbeing in adolescents at risk for substance use and anti-social behaviour. It entails 15 to 20 sessions, in which trained counselors work with both adolescents and parents to develop their communication skills in the family to strengthen family functioning, and help them engage effectively in the interactions within their social network. In sum, these studies suggest that resilience arises from complex pathways among many ecological systems in a person's life and interventions that target change at multiple levels may be beneficial. Multidisciplinary collaborations and participation of important stakeholders, such as the people from school, family, and community-based organisations are needed to optimise the external resources and support in adolescents' lives, and provide them with transferable psychosocial skills to overcome adversities and attain positive developmental outcomes.

\section{Limitations and Future Directions for Research}

This study provided an in-depth understanding of a range of internal and external assets that promoted resilience in South Africa adolescents exposed to violence. However, it is important to note that resilience involves a dynamic developmental process of positive adaptation and is content- and context specific (Jain \& Cohen, 2013). Thus, the findings of our study may be interpreted in light of the specific target population, the environmental contexts, the nature and the level of exposure risk, and the outcomes of resilience. Moreover, due to the qualitative nature of the study, the findings were limited to the description of protective factors that promote resilience. Quantitative studies in this area may help further understand the mechanisms of the processes that lead to resilience - for example, to examine the strength of the effects of the protective factors in reducing the negative impact of violence exposure, and whether different protective factors are interlinked or they interact with risk factors to affect positive adaptation and resilience. Lastly, since this qualitative study is one of the few conducted in South Africa, we sought to enhance the understanding of resilience from a more general perspective and incorporated both emotional and behavioural resilience as our outcome variables of interest. Future studies may adopt domain-specific measures, such as behavioural, emotional, social, and cognitive/educational resilience (Walsh, Dawson, \&
Mattingly, 2010), to inform a more nuanced understanding of resilience among adolescents exposed to violence.

\section{Conclusion}

The aim of our study was to enhance the understanding of the factors that positively enhance the process that leads to resilience in adolescents exposed to violence and to ultimately inform strengths-based interventions. We found that a diverse range of developmental assets across the ecological context may help adolescents overcome the negative challenges of violence exposure and promote healthy psychological functioning and prosocial and adaptive behaviours. More specifically, Some South African adolescents were able to utilise their personal strengths and competencies and draw from the external environmental resources such as social support to adaptively cope with violence exposure, despite living in a violence-prone environment. Interventions may adopt a comprehensive approach and focus on building and strengthening individual coping skills as well as promoting effective and supportive social relations with peers, family, school, and community to optimise the developmental outcomes among adolescents at risk for violence exposure. 


\section{Chapter 7}

Factors that enhance

Resilience in South African

Adolescents living in

High-Violence Communities:

A Qualitative Study on

Stakeholders' Perspectives

and their Challenges 


\section{Abstract}

Parents and teachers are the key stakeholders in adolescents' major living environments (i.e., home and school) who can positively influence their developmental outcomes. This study used individual semi-structured interviews to explore the protective factors that enhance resilience in adolescents living in high-violence communities, from the perspectives of school staff $(n=19)$ and parents/caregivers $(n=17)$. The challenges experienced by the stakeholders while supporting the positive development of their children/school learners were also explored. The findings showed that boundaries and expectations, social support, education and skills building, and constructive use of time can help adolescents attain positive developmental outcomes. Challenges encountered by the stakeholders included dysfunctional home, lack of parenting/support skills, negligence of teachers, ineffective school-parent relationship, stigmatisation of violent learners, and the socio-cultural norm of violence. The findings highlighted that while it is important for adolescents to develop skills and adaptive coping strategies to overcome the negative impact of violence exposure and attain positive psycho-social developmental outcomes, it is also crucial to build skills in key stakeholders to enhance their capacity to support adolescents in violenceprone environments.
Violence is a significant public health concern in South Africa. Adolescents perceive violence as a normal part in their daily lives as it happens frequently (Kaminer, du Plessis, Hardy, \& Benjamin, 2013; Shields, Nadasen, \& Pierce, 2009). They encounter violence directly (e.g., personal and physical experience of violence) or indirectly (e.g., witnessing or hearing about violence) across different developmental contexts, including home, school, and community (Sui et al., 2018; 2019). The results of three Youth Risk Behaviour Surveys in the nationally representative samples highlighted the persistent problem of violence exposure in South African adolescents over the past two decades, such as being threatened or assaulted at school, being approached to join a gang, and being forced to have sex (Reddy et al., 2003; 2009; 2013). Poly-victimisation is also highly prevalent. South African studies have indicated that the majority of adolescents are victims of more than one types of violence and are exposed to violence across multiple developmental contexts (Kaminer et al., 2013; Shields et al., 2009, Sui et al., 2018). Adolescents suffer adverse consequences of violence exposure on their development and functioning (Krug \& Dahlberg, 2002; van der Merwe, Dawes, \& Ward, 2012), including internalising (e.g., depression, anxiety, post-traumatic stress symptoms) and externalising symptoms (e.g., aggressive/delinquent behaviours, tobacco use, substance use, and sexual risk-taking) (Kaminer et al., 2013; Mrug, Loosier, \& Windle, 2008; Shields et al., 2009; Sui et al., 2018). These negative developmental symptoms in adolescents, if not treated, may persist and put them at high risk for poor health and maladjustment in adulthood (Botticello, 2009).

The transition from youth to healthy adulthood is largely dependent on the environment in which young people live, as well as the community and social structure that they are exposed to (Reddy et al., 2011). Although violence exposure serves as a significant threat to adolescents' positive development, other environmental factors may positively shape the adaptation and functioning in adolescents. More specifically, home and school are the two major developmental domains where adolescents spend their time regularly. Both parents and the school staff are the key stakeholders that could play an important role to facilitate resilience in adolescents towards violence.

Resilience is the successful coping with or the overcoming of risk and adversity and the development of competence in the face of severe stress and hardship (McKnight \& Loper, 2002). Researchers in the recent decades have sought to understand the mechanism that leads to resilient developmental outcomes in a context of adversity by identifying the protective factors that can positively influence the process in which adolescents thrive (Fergus \& Zimmerman, 2005). Indeed, not all adolescents suffer the negative developmental consequences and some can positively adjust in the face of violence (Ward, Martin, Theron, \& Distiller, 2007). For example, over half of the adolescents maintained positive psychological functioning years after exposure to violence, and supportive relationships 
with parents and other adults contributed significantly to their resilience (Jain, Buka, Subramanian, \& Molnar, 2012).

Indeed, the positive involvement of parents and teachers can be helpful to promote healthy adolescent development. It may be particularly important for South African adolescents, as poly-victimisation across different developmental contexts is common (Kaminer et al., 2013; Shields et al., 2009; Sui et al., 2018) and can compromise the feelings of safety and security in their living environments. Yet, the development of self-reliance, competency, and the promotion of adaptive functioning are largely built upon a satisfied needs of adolescents' connectedness with significant others (Hogue, Liddle, Becker, \& Johnson-Leckrone, 2002). Thus, parents and teachers are the key stakeholders that can facilitate the connectedness with adolescents and provide a safe and supportive living environments where adolescents can effectively develop competencies to overcome the challenges of violence.

Studies showed that teachers and parents who are attentive and provide adequate supervision and support can promote positive psychological and behavioural adjustment in adolescents living in high-risk environments. For example, connectedness to school (e.g., school belonging, support from peers and adults at school) was associated with less aggressive behaviours in adolescents exposed to violence (Duggins et al., 2016; Perez-Gonzalez, Pereda, Guilera, Abad, \& Finkelhor, 2018). Moreover, high quality parent-child relationship such as felt acceptance by the caregiver (Kliewer et al., 2004) was protective for internalising and externalising adjustment problems in children exposed to violence. Nurturing and responsive parenting style was associated with low levels of antisocial behaviour and high levels of social skills (Vanderbilt-Adriance \& Shaw, 2008). Furthermore, rules and boundaries that discourage violence and promote safety, as well as regular monitoring of adolescents' actions by the adults in the family and school may reduce violent behaviours and substance use and promote the feeling of assurance and safety among adolescents (Duggins et al., 2016; Kliewer et al., 2004; Sui, Massar, Reddy, \& Ruiter, 2020). In addition, parents who are positive role models that discourage the use of substance use and violence themselves may motivate their children to utilise adaptive and non-violent problem-solving skills in conflict resolution with their peers (Sui et al., 2020).

However, several risk factors can pose significant threats to the quality of care and support of teachers and parents. In South Africa, teachers are often subjected to verbal and physical bullying by learners (Woudstra, van Rensburg, Visser, \& Jordaan, 2018), which can lead to negative emotions, disempowerment, low morale, and low motivation of being supportive role players in the school system (Woudstra, et al., 2018). Moreover, one in five women in South Africa had experienced intimate partner violence (Statistics South Africa, 2016), which raises safety and mental health concerns among maternal parents/caregivers in South
African families. Other risk factors such as high rates of substance abuse in South Africa adults (Dada, Burnhams, Erasmus, Parry, Bhana, Timol, \& Fourie, 2015) and single parent households (Meintjes, Hall, Marera, \& Boulle, 2010) have been associated with disrupted family functioning, strained family relationships (Groenewald \& Bhana, 2018), and a general lack of parental supervision and support (Morantz et al., 2013; Raymond \& Zolnikov, 2018).

\section{This Study}

While most literature has quantitatively established the associations of positive influences from home and school with adolescent developmental outcomes, an in-depth understanding of the perspectives of the important stakeholders is needed. This may further the work on the protective factors that promote resilience in adolescents exposed to violence, and help to capture the nuances of their adaptive coping processes. Therefore, this study employed a qualitative approach to explore the strategies parents and teachers use to promote positive psychological, behavioural, and social developmental outcomes in adolescents living in a context of prevalent violence exposure. In addition, we aim to understand the challenges that these stakeholders encounter while helping to support positive development and functioning of these adolescents. Challenges may translate into lessons learnt, and the understanding of both the positive role of stakeholders and their challenges allow researchers to better inform strengths-based interventions to promote resilient developmental outcomes in adolescents living in violence-prone communities.

\section{Methods}

\section{Participants}

Individual semi-structured interviews were conducted by the primary author of this study and two trained research assistants to gain an in-depth understanding of the strategies that the key stakeholders use to help promote positive development and functioning in adolescents. The participants included $n=17$ parents/caregivers of adolescents (male: $23.5 \%$; people of mixed heritage: $52.9 \%$, black: $41.2 \%$, white: $5.9 \% ; M$ age $=44$ years, $S D=11.0$ ), and $n=$ 19 school staff ( $n=16$ teachers, $n=1$ school counsellors, $n=2$ principals; male: $26.3 \%$; people of mixed heritage: $47.4 \%$, black: $10.5 \%$, white: $42.1 \%$; $M$ age $=39$ years, $S D=13.4$ ) of adolescent learners from three schools in Cape Town. The communities in which the parents/caregivers and the schools came from have shown high rates of physical and sexual assault, robbery, murder, property-related crimes, and access to alcohol, drugs, and firearms over the last decade (Urban Safety Reference Group, 2019). 


\section{Procedure}

School staff were recruited in primary and secondary schools located in communities where crime and violence are prominent. The researcher first contacted the principal of the school and obtained permission to invite the staff to participate in the study. Details of the study were then introduced to the school principal, teachers, and school counsellors by the researcher and the ones who showed interest to participate were included in the study.

Parents/caregivers of adolescents were recruited through the schools where the researcher obtained permission to interview school staff. In addition, some parents were recruited in public spaces, such as shopping centres. When recruiting through schools, the researcher obtained permission from the principal to contact parents/caregivers of the learners in school. The parents/caregivers were then recruited through school-parent events, and before and after school when they came to drop off or pick up their children. When recruiting participants in public spaces, the researcher invited parents/caregivers whose children are adolescents (ages between 11 and 19 year old), by approaching the families that were with their adolescent children, or by asking the prospective participants whether they have children who are adolescents. Details of the research were introduced to the parents/ caregivers and the ones who wanted to participate were included.

In the interviews, parents/caregivers first talked about their usual day and their children's home and school environments; school staff talked about their usual day in school, the school environment, as well as the programmes or strategies the school uses to prevent violence and to support the learners who had violence exposure. The participants then shared their perspectives on what an adolescent needs after violence exposure, what they do to support their adolescent child/learner who had encountered violence, what they do to prevent the adolescent from engaging in violent behaviours, and what challenges they have when they supervise and support the adolescents. The interviews with the stakeholders were audio-recorded and lasted between 40 and 80 minutes. They were conducted in a language preferred by the participant (English, Afrikaans, or Xhosa). All 19 school staff interviews were conducted in English. Fourteen parent/caregiver interviews were conducted in English by the primary author and three were in Xhosa by trained research assistants. The interviews were conducted in school or at a mutually convenient location. Each participant received snacks and reimbursement for travel as incentives. All researchers made field notes during and/or after the interview and team meetings were held regularly throughout the data collection process to reflect on the interviews and reach consensus for data saturation. All interview audiotapes were transcribed verbatim. The Xhosa interview audiotapes were first transcribed and then translated into English by a bilingual (English and Xhosa) research assistant, and were cross checked by another research assistant with the same language capability.

\section{Data Analysis}

Using the Atlas.ti software, the interview data were analysed using theoretical thematic analysis based on Braun and Clarke's (2006) six-phase method. The phases were: 1) data familiarisation, 2) initial coding, 3) generating themes, 4) reviewing themes, 5) defining and naming themes, and 6) interpreting and reporting (Braun \& Clarke, 2006).

We aimed to understand the protective factors that enhance psychological, behavioural, and social adjustment in South African adolescents who had exposure to violence. The researchers read each transcript separately first and made notes on the themes that emerged. Codes were created based on the participant's own words and the coding of themes was guided by the "40 developmental assets framework" (Search Institute, 2017). This framework identifies a range of internal and external protective factors that help young people achieve positive developmental outcomes. We used the framework to extract the themes in the interviews while allowing new themes other than the ones listed on the framework to emerge, given that they had theoretical support and empirical evidence in the literature. All three researchers reviewed the codes and agreed upon the main themes that emerged.

\section{Ethics}

The ethics of the study was approved by the Human Sciences Research Council in South Africa (REC 1/21/02/18). Data collection in public schools in Cape Town was permitted by the Western Cape Department of Education (20180504-1777). All participants provided signed consent to participate in the study.

\section{Findings}

Table 1 shows the groups, themes, and sub-themes. The protective factors that positively influenced adolescents' psychological and behavioural functioning from the perspectives of parents/caregivers and school staff were grouped into Group 1 - protective factors. This group consists of four themes, namely, boundaries and expectations, social support, education and skills building, and constructive use of time. The themes on the challenges that the stakeholders encountered when helping to promote adolescent resilience were grouped into Group 2 - challenges, which has three themes, including home-, school-, and socio-cultural challenges.

\section{Group 1: protective factors}

\section{Theme 1a: boundaries and expectations}

Many parents reported consistent parental monitoring to keep their children safe from violence exposure. They kept track of the people and places that their children were in 
contact with and did not allow them to be in potentially dangerous situations. For example, sleeping over at someone's house where the hosting family was not known to the parents was not allowed. Moreover, the parents specifically chose the schools that do not tolerate violence for their children to attend and accompanied their children to and after school to ensure their safety. Not only did the parents ask their children to report regularly on their whereabouts (see quote), they also paid attention to their children, asked about their day, and noticed the unusual emotions and behaviours of their child.

If he doesn't have data, because I want him to be safe, then I will go that extent and say here buy data just to be in contact with him. Not because he needs to have data, but because you want to know what he is doing, where he is. [mother, 49 y.o.]

Table 1. Themes and sub-themes

\begin{tabular}{|c|c|c|}
\hline Domain & Themes & Sub-themes \\
\hline \multirow{4}{*}{ Protective factors } & Boundaries and expectations & $\begin{array}{l}\text { - Family rules } \\
\text { - Family monitoring } \\
\text { - School rules } \\
\text { - School monitoring } \\
\text { - Social role modeling } \\
\end{array}$ \\
\hline & Social support & $\begin{array}{l}\text { - Family support } \\
\text { - School support } \\
\text { - Peer support } \\
\text { - Community support } \\
\end{array}$ \\
\hline & Education and skills building & $\begin{array}{l}\text { - Family education and values } \\
\text { - Family teaching coping skills } \\
\text { - School education and values } \\
\text { - School teaching coping skills }\end{array}$ \\
\hline & Constructive use of time & $\begin{array}{l}\text { - Belong to a programme } \\
\text {. Extra mural activities }\end{array}$ \\
\hline \multirow{3}{*}{ Challenges } & Home challenges & $\begin{array}{l}\text { - Negative family influence } \\
\text { - Lack of effective parenting skills }\end{array}$ \\
\hline & School challenges & $\begin{array}{l}\text { - Stigmatization of violent learners } \\
\text { - Teachers neglect } \\
\text { - Teachers lack skills to intervene } \\
\text { - Ineffective school-parent relationship }\end{array}$ \\
\hline & Socio-cultural challenges & $\begin{array}{l}- \text { Social norm } \\
\text { - }\end{array}$ \\
\hline
\end{tabular}

Similarly, schools had monitoring strategies to keep the learners safe. There were security officers patrolling in and outside school to prevent violence and the dealing of illegal substances. The teachers paid attention to learners' activities in school and made sure they did not engage in peer violence. They noticed the expressions, body languages, and behaviours of the learners who had been physically abused or neglected at home. Cleaning staff assisted the teachers and checked up in the classrooms. In addition, some schools had a file that kept track of each learner's performance and behaviours in school, and documented the unusual signs that the teachers noticed in the learners and their family conditions. This file was important for the teachers to gain a deeper understanding of the learners who were affected by or involved in violence, and enabled the teachers to respond and support with sensitivity.

The other thing we should do as teachers is to monitor the learners, watch them in whatever they are doing. That can help to prevent that one who was going to commit something, because you are there. [teacher, male, 38 y.o.]

Moreover, parental rules were useful to prevent adolescents from engaging in violent behaviours, associating with peers of bad influence, and being exposed to potentially violent environments and violence-related situations, such as substance use. Parental rules were delivered by explaining to their children why these rules are important, or by taking away some of their children's privileges (e.g., watch TV or play with their friend) if they did not follow the rules. Almost all parents set rules for their children to not fight back when someone confronts them and resort to peaceful conflict resolution. Moreover, their children must never walk alone and must be home when it gets dark. They must avoid talking to strangers and never open doors for them. In addition, their children were not allowed to share pictures or videos related to violence to their peers on social media because the parents believed it can create psychological distress in young people and promote violent behaviours. The parents also did not allow their children to carry or play with weapon and sharp object as it can inflict harm to themselves and others.

I think because of the things she has been through, she feels she needs to protect herself, she doesn't want to be vulnerable to anyone. But I always teach her not to fight back, it doesn't make anything better, it escalates things. [mother, 37 y.o.]

Both parents and teachers suggested the importance of school rules to prevent violence among adolescents. Some parents appreciated that the schools created an environment of anti-violence and had disciplinary strategies in place to intervene with the aggressive learners. The disciplinary system was based on the school's "code of conduct" with the aim to help learners understand that violence is not tolerated. Learners could get a warning from relatively minor violent behaviours (e.g., verbal abuse) or a direct detention when involved in physical violence. When the learners received a number of warnings, the school would contact the parents. When the learners significantly disrupted the safe school climate, they would be suspended.

If we didn't have cameras in all the classrooms and outside, if we didn't have our code of conduct, if there weren't consequences which is suspensions and detentions, then the kids will be more physically violent. [teacher, female, 60 y.o.] 
Positive social role modelling from the people in adolescents' major developmental contexts may help them achieve positive developmental outcomes. Some parents suggested positive peer influence is important as their children can be aspired and motivated to achieve in life when they associate with peers who engage in positive activities and prosocial behaviours. Moreover, parents identified the importance of family role modelling. For example, when adolescents were exposed to violence, some parents experienced shock, nervousness, and anger. However, the parents tried to control their own negative emotions and stayed clam because they wanted to focus on caring for their children instead distressing them further. This has enhanced parental support as they put their negative emotions aside and modelled emotion regulation. Furthermore, some parents refrained from engaging in verbal and physical violence with their partner in front of their children. In incidents where the child heard or witnessed their arguments, they apologised to their child for their violent behaviours. As such, these parents delivered the message that violence is not accepted.

I am not the mother that is attacking. I am the mother who will sit down and talk about the situation and try to handle it in a calm way. Bring a calm atmosphere in the house so that the child can say 'now I understand what my mom wants to bring over'. She doesn't want to fight or attack, she wants me to calm down and listen. [mother, 52 y.o.]

Similarly, the teachers showed role modelling by setting positive examples in their interpersonal relationships with colleagues. Many suggested the importance of displaying professionalism in school by being respectful to their colleagues. Even though there were conflicts between some colleagues, the teachers set aside their personal issues and avoided having disagreements when the learners were around. They did that because they wanted to build trust with the learners, just like how teachers should trust one another. Some teachers also avoided punishing learners in front of the class, but instead had a conversation with the learner privately because they did not want to model bullying in class.

I pull them out and I explain to them what they did wrong and why it is wrong behaviour, and then I take them back in class and I explain to the whole class why I took them out and why it is incorrect behaviour... it's better to take them out because else you are doing the same thing, you are bullying the kid in front of the whole class. [teacher, female, 27]

\section{Theme 1b: social support}

Family support was important to help adolescents cope with violence. Some parents availed themselves to care and support their children who were exposed to violence. They suggested that quality family time (e.g., family outings) was important to strengthen the bond with children and help them cope with violence. They wanted their children to know that family is their safe haven and they can always trust and rely on the family. Furthermore, the parents encouraged their children to express themselves and openly speak to them about the victimisation experience. The open communications helped the parents understand what their children were going through so they could provide more timely and targeted support. The parents displayed understanding and non-judgmental attitude when speaking to their children, and refrained from blaming the child for their responses to violence even if they used substance to cope. Many parents also referred their children to a counsellor and spoke to the teacher or the perpetrator's parents directly when their children experienced violence in school.

They will communicate with me because they know they won't be judged and not blamed. I comfort them and I support them, because if something happens they will be scared to speak to you because you shouted at them before, so it means they will go through hurt and pain all alone and maybe resort to doing the same thing to others. [mother, 37 y.o.]

School support was evident and the teachers availed themselves to support the adolescents who had violence exposure. For example, each teacher was responsible to mentor a group of learners and some teachers sacrificed their break time to talk to the learner. Since some learners were at risk for violence victimisation and violence perpetration, and were not getting adequate care and supervision from home, many teachers built a close and caring relationship with the learners and encouraged open communications. This relationship is beyond the traditional teacher-learner relationship where academic work is largely focused, and they spent additional effort and time to attend to the learners' personal and emotional needs. The teachers called learners "my kids" and described themselves as not just teachers, but also "parents and social workers and counsellors in some way". Moreover, school was the only safe environment for many learners, and the teachers believed it is crucial to create a violence-free, learner-friendly, and family-like school climate to assure learners that they can feel safe and respected.

I've had learners live in my home for short periods of time, if I know a learner is really struggling and capable of offending. They can come to me over the weekend, just to get away from that kind of environment. [teacher, female, 45 y.o.]

When the learners had trouble learning due to psychological distress, or when they engaged in violent misconduct, many teachers demonstrated sensitivity and empathy towards them. Instead of blaming and disciplining these learners, the teachers understood that some learners' family environment was a trigger of their poor academic performance and violent behaviours because many lived in violent homes and experienced lack of family support 
and supervision. They empathised with the learners and were not judgmental towards their behaviours. They helped the learners to overcome the personal challenges by giving them additional guidance to complete their school work and referring them to the school counsellor for further support. These teachers believed a learner's developmental wellbeing is more important than academics.

Our role is always to first try and help the learner with what they are going through... It's about making sure that the learner feels happy here, because you spend so much time of your day at school. [teacher, female, 45 y.o.]

In addition, the schools collaborated with other sectors to support the learners. For example, some schools were connected with social service institutions where the learners could go for drug counselling if they had substance abuse, or psychological counselling if they needed mental health support to cope with violence exposure. The schools worked with the Department of Education on the safe school project, where the teachers could contact qualified social workers to do home checks and provide counselling service for learners who experienced abuse at home. One of the schools has liaised with the police and a police officer visited the school regularly to monitor and ensure safety.

Moreover, parents and teachers suggested the need to have peer support for adolescents exposed to violence. Peer support can make the adolescents feel that they are not alone and such support may occur more naturally due to the similar age and life experiences of adolescents. It may also aid in teachers' support for the victimised learners as the teachers were sometimes unaware of the violence in school and could find out through the peers of the learners.

We might not pick it up immediately in the class that a child experienced violence... they will rather open up to their peers instead of us. The peers will spend more time with the child, or the prefects are there and the child can trust the prefects. If the prefects feel they can deal with the situation then they can sort it out, or they can report to us. [teacher, female, 32 y.o.]

Many parents mentioned that a caring community can help keep adolescents safe. They believed the families in the community should collectively support and take care of each other's children to prevent them from being exposed to potentially risky environments. Some communities had "neighbourhood watch" that patrolled in the community and updated parents about the crime and violence-related incidents that happened in the community. A connected community encouraged the adolescents to seek support when their parents were not immediately available.
In the community we should always be seeking out for things and people that the kids feel they can go to the people they trust. We need to make friends and spend time in one another's homes to build trust. [mother, 33 y.o.]

\section{Theme 1c: education and skills building}

Many parents believed that education starts from home and prosocial values were taught to their children so them can grow up as socially responsible individuals. They talked to their children about the concept of bullying and being bullied. They explained why violence is not a good thing and not the solution to disagreements, and they discussed the consequences of violence.

Furthermore, many parents identified the need to teach adolescents adaptive coping skills to cope with violence exposure. Some suggested that adolescents should develop personal boundaries, self-confidence, and assertive skills to reject negative social influences such as gangsterism, delinquency, and substance use. Other parents believed that adolescents must learn anger management to prevent violent behaviours. Self-defence was also mentioned as an important skill to protect themselves, especially for girls. To regulate emotions, some parents suggested their children to listen to music or write about the negative feelings if they were not comfortable to speak. Some told their children not to ruminate about the incident of violence because that can build up more anger. Some taught their children strategies to stay away from violent situations, such as being vigilant of their surroundings and walk away, and knowing how to seek support from an adult they trust. In addition, the parents told their children to keep a positive attitude and believe that they have the self-efficacy to overcome the challenges of violence exposure.

My daughter at first didn't like to talk about negative stuff or her feelings. I told her to write down her emotions, how she feels, rather speak to a paper. She will write it down and keep it to herself and when she is ready she will either burn it or destroy it. It helped her in the sense that now she can sit me down and tell me that she needs to tell me something, something bad has happened. [mother, 37 y.o.]

The schools conducted interactive workshops to educate learners about violence. They invited police officers, people from social service, and motivational speakers from the local communities to teach learners about gang violence and substance abuse, create awareness of the social conditions in the local communities, and promote them to engage in prosocial activities. School counsellors assisted the teachers and ran anti-bullying workshops. Moreover, in life orientation lessons in school, the concepts such as bullying, grooming, assault, and stereotyping and discrimination were explained to the learners with examples. These information allowed learners to gain a deeper understanding of different types of 
violence and the associated negative consequences. The teachers also taught learners to appreciate cultural diversity and respect one another's differences to prevent peer violence.

Furthermore, many schools educated learners about prosocial and moral values, such as peace, respect, responsibility, and cooperation. These core values were incorporated in the daily teaching curriculum and were emphasised in the school environment such as displaying them on the wall and having senior learners wear badges of these values. The national holidays such as women's day and Mandela day were used as opportunities to emphasise these values, such as respecting females and being kind. Many teachers believed that they have the responsibility to educate learners positive values to promote their prosocial behaviours because many of them come from dysfunctional homes (e.g., single parenthood, violence, parental drug abuse) where they are unable to receive adequate guidance and support from the family.

At school we teach them how respect, like if something in class happens, name calling for example. Then I will ask them is this according to the value of the month? Then they will tell me that was disrespectful and explain to me why. You need to remind them because at home they don't have those role models that can teach them. [teacher, female, 27 y.o.]

Moreover, some teachers mentioned a positive discipline approach to teach learners that violence is not tolerated. Instead of the traditional punitive approach where discipline was given to aggressive learners without much explanation of the connection between their behaviours and the discipline, this approach helped the learners reflect on their behaviours and understand the consequences of violence on themselves and others. For example, the teachers gave an opportunity to each learner who was involved in a physical fight to voice their views in front of each other and have a discussion about how they made the other person feel. The focus on each other's feelings elicited empathy from the learners who perpetrated the violence, made them aware that their behaviours had a negative impact on others and thus became more motivated to prevent future use of violence.

The only way you can prevent violence is to teach the perpetrator what harm they causing to other people... Then in the future when they face a similar situation they are less likely to be violent because they know what suffering they can cause... That's the principal I am getting the staff to use, to not just punish but to have the conversation, to rebuild the relationship between the victim and the perpetrator. [principal, male, 43 y.o.]
Teachers also helped learners to build adaptive strategies to cope with violence. They reminded learners to utilise strategies to protect themselves from violence such as being wary of the surroundings and noticing personal discomfort and warning signs of danger. They also provided guidance on the strategies that help refrain from violence perpetration, such as anger management, walking away from the situation, and knowing ways to report to an adult when there is violence. Furthermore, the teachers coached the learners to explore different ways to resolve peer conflicts in a peaceful manner. In addition, some learners who engaged in violent behaviours were sent to the school counsellor to constructively learn problem solving and interpersonal skills.

I always tell them to try to remain calm. If you feel like you want to punch someone, rather just take a moment and step out. Like run out of my classroom. Take a walk, I will come find you and we can sort it out. [teacher, female, 25]

\section{Theme 1d: constructive use of time}

Many parents suggested that constructive use of time such as attending religious gatherings at the community church, participating in community events, and being a member of a club may positively influence adolescents' development in a context of high violence exposure. These activities outside home were believed to strengthen adolescents' interpersonal skills as they learn to interact with different people. Parents also thought that their children could experience a sense of belonging in the community and feel supported by the people around. Similarly, both parents and teachers believed that sports could strengthen an adolescent's ability to socialise with others and build effective interpersonal relationships. Through playing team sport, adolescents can learn to work with different personalities and become respectful towards others.

Moreover, the schools had extramural programmes including singing, sport, and math Olympiad. There were also events such as an entrepreneur day that let learners sell things and food they make, a talent show that provided a platform to showcase learners' skills, and a job shadowing event that let learners explore their interests and stay motivated in life. Spending time on activities that the learners were good at and interested in, helped to shift their focus on something positive and constructive, explore their talents and skills, and enhance self-esteem and selfworth. Prosocial behaviours can also be motivated, as a teacher mentioned, social outreach events such as community service has given the learners opportunities to be serving and kind to others, which reduced the prevalence of aggression in learners.

The naughty kids at school were serving homeless people. They were engaging, and I really saw a different side to them. At the end of the eight weeks, not all fifteen had changed their behaviour completely, but there were a handful that had stopped the violent, aggressive behaviours at school. [school counsellor, female, 26 y.o.] 


\section{Group 2: stakeholder challenges}

\section{Theme 2a: Family challenges}

Parents and teachers discussed that one of the biggest challenges from adolescents' homes is a dysfunctional family, these include divorced parents, abusive parents, overcrowded home environment, domestic violence, and parental substance abuse. Some parents were coping with their own life adversities and could not fully attend to the adolescents' developmental needs. The teachers discussed about their frustration to reach out to certain learners due to their violent home circumstances and some parents kept their children away from attending school. Both teachers and parents believed these influences from home negatively shaped an adolescent's development, putting them at risk for psychological distress, delinquency, and bullying. For example, the adolescents who witnessed domestic violence may learn that this behaviour is normal and practice it in school, or as a way to seek attention due to lack of adequate supervision from home. These learners may not have the knowledge and skills for effective communication and conflict resolution as they practice what they see at home.

They bring that attention seeking behaviour to school, because sometimes there is violence involved, neglect involved in the families. Then they bring that behaviour to school as an outcry for help. [teacher, female, 32 y.o.]

Many parents were aware that adolescents are risk taking, easily influenced by peers, and are impulsive and disobedient. As such, they expressed that open communication with their children was not always possible. Moreover, due to the challenges associated with negative family functioning experienced by many parents such as divorce, some expressed their frustration towards raising an adolescent child, especially as a single mother. Many of them were clueless about effective parenting, and did not have enough time and energy to take care of the children as a single mom. Some questioned their parenting style such as blaming themselves for being too lenient. They felt helpless because they were often subjected to verbal violence by their own children. The parents suggested that it is important to attend workshops to learn about strategies that can keep their children safe as well as effective parenting skills, so that they can be equipped to promote positive development of their children who may be at risk for violence exposure and violence perpetration.

There is a child in every divorce who just never recovers from the divorce and they always blame the caregiver, the mother. Especially if it's a mommy with sons who aren't happy. That can be threatening. We become very scared, we become calm and just say what the child wants to hear. [mother, 54 y.o.]

\section{Theme 2b: School challenges}

Stigmatisation happened in schools where some teachers did not spend adequate time to get to know the learners who showed externalising symptoms such as being aggressive towards others, but instead simply label them as "bad learners". They did not give much attention and support to those learners and did not spend time on getting to understand the root trigger of these learners' behaviours. Stigmatisation can promote more delinquent behaviours as the learners feel judged and hopeless, and behave out of frustration.

The teachers just think that he [the learner] is rude, and when I asked him like why he behaved that way he was like: everybody thinks I'm this way, I'm a gangster, so I might as well act this way. [school counsellor, female, 26 y.o.]

Some teachers said that their colleagues were neglectful and did not respond to the incidents of violence in school or until the incidents were reported. Teachers' neglect promoted more peer violence in school and made the victimised learner feel unworthy. Those teachers did not involve in responding to school violence due to the fear of being personally victimised. Many said that they were sometimes afraid of the learners because they can be violent and bully the teachers. Some learners were also involved in gangsterism, which can be particularly dangerous to the people who intervene. The teachers said that society often overlooked the safety needs of the teachers. The fear has prevented them from attending to incidents of violence in school as they did not want to get involved to protect their personal safety.

I don't think they put more effort in it because the children bully the teachers also. They also need to protect themselves. They will keep quiet and not get involved. [teacher, female, 22 y.o.]

Given the magnitude and intensity of violence exposure in South African adolescents (e.g., rape, gang violence), responding to these types of violence can take an emotional toll. As such, the teachers identified the need learn how to handle violence without experiencing strong negative emotions such as shock, fear and sadness. They believed counselling for the teachers is important as many of them went through trauma from hearing/witnessing violence experienced by learners, and some had difficulties regulating their own emotions when having to respond to the incidents of violence in school.

Moreover, some teachers complained that they had a busy academic schedule and therefore they did not have time or energy to respond to the incidents of violence in school. They believed academic should be the primary focus and they were under pressure to attend to both teaching and support for victimised learners. It was impossible for them to monitor 
and support every learner in class. The teachers expected other professionals such as the school counsellor to share the responsibility and follow up on the learners' wellbeing and functioning.

The teachers just don't have time for extra stuff. So then you maybe get other people to do it because there are so many subjects, so much work and so many kids in the class. Like now, very rushed, just get to the very necessary things. No time for building relationships. [teacher, female, 60 y.o.]

Furthermore, some teachers said that they lacked skills to respond to the incidents of violence in school. They felt helpless because despite teaching learners positive values and incorporating disciplinary strategies to enhance awareness for anti-violence, some learners still repeated violent behaviours. The teachers also believed that they were not qualified to provide psychological service for the learners who had anger problems and they expressed the needs to learn counselling skills to support the learners. Other important skills needed by the teachers were communication skills with the victimised learner; gender differentiated approach to respond to violence; ability to look for signs in learners who may need support; and the understanding of different types of violence to gain more insight into the personal experiences of victimised learners. These skills were believed to enhance the support for the learners and build effective relationship with the learners.

In every school you going to find a child being raped, or abused or maybe strangled. It is difficult where you are emotionally involved all the time. You feel sorry for the children or you get very angry at the child that swears at you, that throws you with stuff, hits you or spits at you. They should definitely have a way where they train these teachers with some workshops, like regulating the teachers' emotions, about really working with broken children. [principal, female, 48 y.o.]

School-parent relationships were not always effective. Some teachers felt under pressure that they were expected by the parents to be solely responsible to ensure safety, positive functioning and performance of learners, yet the parents were uninvolved in school. Despite the open door policy in schools where parents were welcomed to see the teachers and principal, some parents never came to school events and meetings. The non-involvement was often due to the parents' substance abuse, which prevented them from taking interest in their children's lives. In addition, when a learner engaged in violent behaviours, the teachers were blamed and subjected to verbal violence by the parents. These parents responded defensively and were not open to teachers' perspectives when the teachers contacted them to discuss about their children's experiences and performance in school. Some were overprotective of their violent children and thought they were not at fault. Some abused their children at home but did not want to change their abusive parenting style. Some parents were going through their own personal issues (e.g., substance abuse, violence) and refused to deal with the similar problems experienced by their children. These refusal and defensive attitudes of parents have led to inefficient communication between the school and parents, which impeded the teachers to find effective solutions to promote learners' functioning and adjustment.

I asked the parent, "Do you know your child is living alone? He is 10 years old and living alone with his dog." She said I seemed to be more concerned about her child than she is. I said yes apparently I am. Then she just started swearing at me and I put the phone down. [principal, female, 48 y.o.]

\section{Theme 2c: socio-cultural challenges}

The social norm of violence was reported as a significant factor that promoted adolescents' externalising behaviours. The negative social influence was evident in peers of adolescents who engaged in violent activities and perpetuated the idea among young people that violence is acceptable. Furthermore, there were high rates of crime and xenophobia in the communities, and gangsters offered adolescents cigarettes, weapons, and drugs, and involved them in delinquent activities. As a response of anger, people in the communities often resorted to violence against the ones who perpetrated violence. Since violence was highly prevalent, the police was under immense pressure to intervene. Some parents said the police had a bad reputation because they hardly responded promptly to the reported violence. The personal safety of the police was sometimes threatened if they intervened the crime, and not all police could be trusted as some were involved in illegal drug dealing activities. As such, adolescents are growing up in a social norm of violence where the people in the community and the peers they know engaged in violence and violence-related behaviours (e.g., substance use)

This year the grade 7's are very violent. It just reminds me of some game they play called $V A N D A L$. I come to realise that the word comes from vandalism. It's gangster things. The Vandals are just in the habit of destroying things, property. [teacher, male, 39 y.o.]

Moreover, the social norm of violence is deeply entrenched in cultural values. In some culture, it is an acceptable corporal punishment for parents to beat their children if they disobey. Some teachers had to stop the parents from beating their child in the school parent meetings when the parents found out the child had a misconduct. Violence is also rooted in the political history in South Africa where it was highly practiced to gain political power, which has shaped some parents' perception that violence is tolerated and an effective way to solve problem. 
We think we can solve things through violence. Most of African countries we got our independence through violence. So we think everything can be solved through violence. [mother, 50 y.o.]

\section{Discussion}

This study explored stakeholders' (parents/caregivers and school staff) perspectives on the factors that may positively influence psychological, behavioural, and social developmental outcomes in South African adolescents living in high-violence communities. We found that boundaries and expectations, social support, education and skills building, and constructive use of time were protective factors. Stakeholders' perspectives on their challenges associated with responding to the needs of these adolescents were also explored.

In our study, boundaries and expectations, specifically rules set by the parents and school staff and consistent monitoring may prevent adolescents from violence exposure, associating with peers of bad influence, exposing themselves in potentially dangerous environments, and engaging in violence and violence-related behaviours (e.g., substance use, carrying weapons, sharing content of violence on social media). The use of rules to promote positive behavioural functioning has been found in another study where the adolescents from families with "strict" alcohol rules had a lower level of binge drinking than the ones from families with lenient rules (Østergaard, Järvinen, \& Andreasen, 2018). In our study, the rules from home and school were often used in combination with a disciplinary strategy if the adolescents failed to comply, e.g., school detention systems, with the aim to help adolescents learn from their misconducts and adhere to prosocial behaviours. Moreover, we found that consistent monitoring allowed parents and school staff to timely support the victimised adolescents when they noticed unusual emotions and behaviours that disrupted adolescents' daily functioning. These monitoring strategies may allow the stakeholders to be sufficiently informed of the adolescents' lives and undertake appropriate action when necessary, and help the child successfully navigate through the developmental period to make safe and responsible decisions (Keijsers, \& Poulin, 2013).

In addition, positive social role modelling from peers with positive influence, or parents and teachers who model emotion regulation and practice respectful and non-aggressive interpersonal relationships, created a social norm that prosocial behaviours are expected. Individuals are nested within relational and larger socio-political contexts. The social influences dictate culturally acceptable and unacceptable behaviour and can shape the development of the adolescents, possibly through a process of social learning via direct observation and socialisation (Bandura, 1977). Other studies have also shown that positive social influences promote healthy and adaptive functioning in adolescents. For example, emotion regulation skill in caregivers reduced internalising and externalising adjustmen problems associated with violence exposure in their children (Kliewer et al., 2004), and lower perceived peer alcohol use was associated with reduced use in adolescents (Gersh, Lee, \& McCarty, 2019). Conversely, in our study, we found that one of the challenges that prevents positive development in adolescents is the prevailing negative cultural and social norm that promotes the use of violence to resolve conflicts and for corporal punishment. Substantial studies have indicated that witnessing and growing up in an environment of violence may increase adolescents' risk for psychological problems (Sui et al., 2018) and aggressive and delinquent behaviours (Choe, Zimmerman, \& Devnarain, 2012; Kaukinen C, Buchanan L, Gover, 2015). Since socialisation is the process through which individuals acquire beliefs, values, social norms, and practices that allow them to successfully interact with society, adolescents' aggression and violence may be a result of an incorrect and misrepresented belief through which they perceive that others in their social group believe the use of certain behaviours is acceptable (Berkowitz, 2013).

In line with other studies (Duggins et al., 2016; Kliewer et al., 2004; Pérez-González et al., 2018), we found that social support from home, school, peers and community may buffer the stressful experience of violence victimisation, and facilitate adolescents' adaptive coping with violence exposure. These social support structures create a secure platform from which adolescents can meet their needs of connectedness with others, and feel safe and guided by the significant others in their major developmental contexts to build psychosocial competence and engage in developmentally healthy activities (Hogue et al., 2002). In particular, many school teachers not only availed themselves to support, they also established a caring and understanding relationship with the learners, which is beyond the traditional teacher-learner relationship where only academic performance is the focus. This close relationship was deemed necessary by the teachers, as many adolescents came from dysfunctional homes where there were lack of interest and support from the family. This finding emphasises the importance of school support, particularly in the South African context where parental substance use and single parenthood are highly prevalent (Groenewald \& Bhana, 2018; Meintjes et al., 2010). Thus, if support from the direct family developmental context is depleted, support from other contexts may be crucial to help adolescents develop competencies to cope.

However, our results also indicate that due to the dysfunctional home environments in many adolescents' families as well as single parents' lack of skills (and time) to provide adequate supervision and support for their children, school teachers felt under immense pressure to not only adhere to the academic curriculum but also support and attend to adolescents' personal needs and wellbeing. This difficulty is compounded by the dangerous nature of 
some incidents of violence and the learners who are violent, which added to some teachers' reluctance (due to fear) to support and respond to incidents of violence in school. Teachers' non-involvement may, as indicated in another study, further increase the risk of violence victimisation among adolescents (Marachi, Avi Astor, \& Benbenishty, 2007).

As shown in our study, the support for adolescents can be further hampered due to a compromised parent-school relationship that lacked trust and involved poor communication. There is a need to strengthen parent-school relationships so both stakeholders share the responsibility to promote positive adolescent development. Studies have shown that an effective relationship between teachers and parents may improve school climate, and strengthen collective efficacy to support and supervise the adolescents. For example, parent volunteering and serving on a committee in school is associated with less school crime and violence (Hamlin \& Li, 2019). Moreover, teachers' positive perceptions of a child's parents are associated with the quality of the relationship with learner: A closer and less conflictual teacher-child relationship may promote children's socioemotional functioning, including emotion regulation, the ability to form and maintain relationships, and effortful control of externalising behaviours (Jeon, McCartney, Richard \& Johnson, 2020).

While parents indicated they incorporated life skills education through conversations with their children about violence and taught them adaptive strategies to cope with violence, the schools were able to complement these skills and education from home, and provided more structured and systematic strategies. These included the education of positive moral values through organising guest speakers, anti-violence workshops, and Life Orientation lessons. These all help adolescents gain knowledge about different types of violence, as well as acquire skills for emotion regulation, and social and interpersonal skills that refrain them from engaging in violence and violence-related activities. As shown in the literature, the ability to regulate sadness and anger, is associated with better psychological outcomes in adolescents exposed to violence (Kliewer et al., 2004) and may buffer the impact of violence exposure on adolescents' health risk behaviours through resorting to adaptive strategies to cope with negative emotions (Sui et al., 2019). Moreover, social skills may help the adolescents have realistic expectations of social situations and become adept at reading social norms, facial expressions, and intention of others, and thus establish more self-control and engage in effective problem-solving in high risk situations (Holmes, Yoon, Voith, Kobulsky, \& Steigerwald, 2015).

In particular, some teachers used "positive discipline" to teach prosocial and non-violent behaviours to adolescents. As opposed to the traditional disciplinary system where learners who had a misconduct had to obey an instruction as a consequence of their behaviour (e.g., have parents called in, suspension from school), positive discipline focuses on eliciting empathy from perpetrators of violence to help them recognise the hurt and harm they have inflicted on others, thereby prevent future violent behaviours and strengthen positive socia relationships with others. Indeed, behaviours that are motivated by adolescents' concern for others are thought to reflect optimal social functioning and prosocial behaviours. Empathetic and perspective taking ability represents a key factor in adolescents' psychosocial maturity and developmental transition, and is associated with greater adherence to prosocial behaviours (Brittian \& Humphries, 2015), such as less involvement in bullying others and more active helping of victimised peers (Gini, Albiero, Benelli, \& Altoe, 2007). A study found that positive disciplinary practices used by parents that involve verbal reasoning and explanations to enhance the child's awareness of his or her consequences to themselves and others may elicit empathy and foster an understanding of the perspective of others, and in turn motivate their prosocial tendencies. The mechanism of the association between high levels of empathy and lower aggression, may be partly explained through the process of decreased moral disengagement, by converting harmful behaviours to acceptable ones and making allowances for the enactment of antisocial and immoral conduct (Wang, Lei, Yang, Gao, \& Zhao, 2017). On the other hand, the traditional disciplinary system in school and in family may seem to involve power assertion where strong actions to adolescents' transgressions were used to establish the parents or teachers as the ultimate authority figure with little or no explanation. The adults who use such practices often apply corporal punishment, and are considered inflexible and overly demanding (Carlo, Knight, McGinley, \& Hayes, 2011).

Lastly, we found that constructive use of time of adolescents, including belonging to a community and religious programme, engaging in extramural activities (e.g., sport), and participating in school events that focuses on developing positive moral values and aspiration were important to facilitate positive psychological and behavioural functioning in adolescents. The positive developmental activities allowed the adolescents explore their talents which may foster self-efficacy and self-worth. In addition, adolescents can learn interpersonal and communication skills that decode social situations, and understand the thoughts and feelings of self and others as they meet and interact with different people. The positive socialisation may protect them from engaging in aggressive behaviours (Kewalramani \& Singh, 2017) and reduce the risk for psychological harms of violence victimisation (Lepore \& Kliewer, 2019). Also, the extramural activities after school have been found to promote quality family time and family bonding when the parents are involved in these activities with their children (Whitson, Robinson, Valkenburg, \& Jackson, 2019).

\section{Implications for Intervention Development}

Schools can be a major site for interventions as more systematic and dynamic programmes and activities can be provided to a large number of adolescents, providing opportunities to positively shape their developmental processes (Gevers \& Flisher, 2012). Given the unique context of South Africa where violence exposure is highly prevalent in adolescents' 
major developmental contexts, including home, school and community, it may be crucial to build on the prime intervention site of school and incorporate a multi-setting and multicomponent intervention to extend the positive influences that promote healthy psychosocial development in adolescents living in high-violence communities.

In our study, some parents and teachers felt that they did not have adequate parenting skills and personal competencies to support adolescents, as they lacked the skills to respond and support when the incidents experienced by the adolescents were too violent, or when the adolescents refused to listen to them. Thus, there is a need to provide skills training for parents and teachers to understand adolescents better and adequately respond to their needs in a context of high violence exposure. Moreover, skills training to enhance personal competencies in parents and teachers, such as emotion regulation in the face of conflicts and interpersonal skills, may enhance the support process and demonstrate positive role modelling to adolescents for prosocial behaviours (Kliewer et al. 2004; Sui et al., 2019). In particular, teachers in South African schools should be psychologically supported, as they are exposed to violence vicariously through responding to the violent incidents in school, and are sometimes subjected to violent acts directed at them by the learners.

Education about violence and the skills to adaptively cope with and refrain from violence may be helpful in school through varied programmes and constructive activities to keep adolescents away from the streets and help them develop self-esteem, interpersonal skills, resistance skills, emotion regulation, problem-solving, and empathy and perspectivetaking. These skills, as evident in our study, may reduce violence exposure and violence perpetration, and promote psychological functioning and adaptive coping behaviours in response to violence. Studies have indeed found that school programmes that provide skills and competency building may be associated with positive psychological and behavioural outcomes in adolescents. For example, Social Skills Training (Vahidi, Fathi, Hosseininasab, Moghadam, \& Kiani, 2007) is an in-school intervention where trained teachers teach children a diverse range of skills over 11 sessions, including self-knowledge, empathy, interpersonal communications, responding to social interactions, initiating social interactions, coping with emotional issues, and maintaining social interactions and social problem solving. The skills building was delivered through positive reinforcement (i.e., attention and praise), role play, feedback, and behaviour rehearsal, and has shown effectiveness in reducing aggressive behaviours in children (Vahidi et al., 2007). Other skill building interventions in school have also shown to reduce violence-related behavours such as substance use (Das, Salam, Arshad, Finkelstein, \& Bhutta, 2016; Griffin, Botvin, \& Nichols, 2006). Similarly, Coping Power (Lochman et al., 2009) is delivered by trained school counsellors with the aim to build skills in both adolescents and parents, and has shown to be effective in reducing adolescents' minor assault (hitting or threatening to hit others) two years post-intervention.
It involved enhancing problem solving, peer refusal, conflict management, coping and social skills in adolescents as well as parents' ability to affective (e.g., warmth and bonding to children) and instrumental parenting practices (e.g., consistent and appropriate monitoring, supervision, and reinforcement of behaviours). Akin to these intervention, it may be helpful for interventions to priorities skills training for key stakeholders (e.g., teachers and parents) to enhance the support process for positive adolescent development, as well as building transferable skills and competencies in adolescents for them to overcome the challenges of violence and achieve resilient developmental outcomes.

\section{Limitations and Future Directions for Research}

While this study is one of first conducted in South Africa that contributed to an in-depth understanding of the protective and challenging factors that enhance positive psychosocial outcomes in South Africa adolescents living in high-violence communities, several limitations and recommendations for future research may be noted. Since this study is qualitative in nature, quantitative studies in this area may complement the understanding of the mechanisms of the processes that lead to adolescent resilience. These may include examining the strength of the relationships between variables and include domain-specific outcome measures, such as behavioural, emotional, social, and cognitive/educational resilience (Walsh, Dawson, \& Mattingly, 2010), to provide a more refined understanding of the protective factors on positive youth development in the context of high violence exposure. Moreover, the findings of this study were solely based on the perspectives of parents/ caregivers and school staff. Although it provides a focused and in-depth understanding, it may be beneficial to interpret the findings in combination with research that is based on adolescents' perspectives to grasp a more comprehensive view. Lastly, the ethnicity of the participants was predominantly black and mixed heritage, and the predominant gender was female. Although these individuals are at most risk for violence exposure in South Africa (Kaminer et al., 2013; Sui et al., 2018), caution may be applied when interpreting the findings in the current study, as they may pertain to the particular cultural-, gender-, and social norms and practices in the responses to the needs of adolescents living in highviolence communities. 


\section{Chapter 8}

\section{General Discussion}


This $\mathrm{PhD}$ project was motivated by the strong interest and need to understand one of the biggest public health concerns in South Africa: violence. South Africa is a fledgling democracy and in the post-Apartheid era (which ended 1994), the society is undergoing rapid socioeconomic transition. In the contemporary society, violence and crime remain high, and the prevalence is compounded by the availability of illegal intoxicating substances, alcohol, and weapons in the communities, as well as deeply entrenched social inequalities that stemmed from the political history of South Africa.

Adolescents in South Africa are at high risk for direct and indirect violence exposure and a significant proportion of them engage in violence perpetration. As argued in this dissertation and elsewhere (e.g. Botticello, 2009; Das-Munshi et al., 2016), adolescents disproportionately suffer the negative consequences of violence, as the experiences of victimisation and perpetration can take a significant toll on their psycho-social development during adolescence, and may lead to long-term impact on the health and functioning in adulthood. Adolescents are in a critical developmental period where their psychological, behavioural, and social domains of development are - partially but substantially - being shaped by the environment they live in. Indeed, growing up in an environment where violent crimes are prevalent, and in combination with other social problems related to substance use, inequality, criminality, and neglectful or abusive parenting, South African adolescents are at risk for a vicious cycle of violence victimisation and violence perpetration, and poor functionality throughout their lives. Given the unique historical and social context in South Africa, the aim of the research reported in this dissertation was to understand victimisation and perpetration experiences among adolescents as well as the associated correlates, to inform targeted intervention development and ultimately promote positive development and adjustment in South African adolescents.

This final chapter integrates and discusses the results of all six empirical studies that together constitute a needs assessment of violence victimisation and violence perpetration in South African adolescents. A combined approach was taken to summarise and to interpret the findings to highlight the commonalities and/or differences across the dissertation. This chapter further reflects on the strengths and weaknesses of these studies, and outlines the practical implications of our findings.

\section{Prevalence of Violence Victimisation and Violence Perpetration in South African Adolescents}

Chapter 2 provided an overview of the prevalence of four violence typologies in three nationally representative samples of South Africa adolescents over the three survey years of the Youth Risk Behaviour Survey (YRBS, 2002, 2008, 2011). The results showed that on average, just under a third of (30.8\%) adolescents had non-involvement in both victimisation and perpetration, a similar percentage were victims only (29.8\%), only $9 \%$ were perpetrators only, and $24.6 \%$ were victim-perpetrators. Moreover, between 2002 and 2011 , there was a significant increase in the prevalence of non-involvement in adolescents, a significant decrease in the prevalence of victims only and victim-perpetrators, and no significant changes in the prevalence of perpetrators only. These findings suggest that the majority of South African adolescents thus had experiences with violence: in victimisation only, perpetration only, or both, between 2002 and 2011.

Although the prevalence reported in Chapter 2 showed decreases for some violence typologies, unfortunately, later YRBS data are not available to inspect more recent trends, and the magnitude of victimisation and perpetration in South African adolescents should not be neglected. As reflected in more recent national surveillance studies, over half of the South African adolescents reported having witnessed someone in their community using threat or force to physically harm another person (Leoschut, 2009), and that 22.2\% reported they had been threatened or had been the victim of an assault and robbery in school (Burton \& Leoschut, 2013). Almost one in five of the adolescents in Leoschut's study had considered committing what they knew would be a criminal offence and over half of these young people had not only considered this but had already committed such acts (Leoschut \& Burton, 2006).

Another important observation in Chapter 2 is the overlap in the experiences of victimisation and perpetration within the same South African adolescents, with an average proportion of almost $25 \%$ of adolescents in three combined nationally representative samples. Indeed, literature has established an association between exposure to violence and subsequent violent behaviours in the same individuals (Jeong, Davis, \& Han, 2015; Reingle \& MaldonadoMolina, 2012). One possible mechanism for the overlap of these experiences may stem from a socialisation process (Bandura, 1977), in which adolescents acquire violent behaviours through social learning and internalising it as the 'norm' to solve problems. It is likely that this may be part of the explanation, particularly in South Africa where violence exposure is endemic and the country only abolished a long history of political violence less than three decades ago. 
In Chapters 3 and 4, we zoomed in to focus on the adolescents from the Western Cape Province, where rates of murder, sexual assault, and robbery are highly prominent (Urban Safety Reference Group, 2019). The total number of exposures to different incidents of violence victimisation, i.e., poly-victimisation, was high in both studies in Chapter 3 and 4. For example, all adolescents in the sample in Chapter 3 reported to have experienced at least one incident of victimisation (actual range $1-20$ incidents). These findings indicate that the majority of South African adolescents experience multiple and different types of violence victimisation across multiple contexts, a finding that has been confirmed in other South African studies (Leoschut \& Kafaar, 2017; Kaminer et al., 2013). One danger of the extensive exposure to different types of violence is emotion desensitisation, when adolescents become used to victimisation experiences and perceive it as a normal part of daily life (Shields, Nadasen, \& Pierce, 2013). Such desensitisation has been found to contribute to adolescents' violent behaviour due to reduced level of remorse and empathy for others (Mrug, Madan, \& Windle, 2016) - creating a vicious cycle of violence victimisation and perpetration.

\section{Victimisation and Perpetration: Sociodemographic and Protective}

\section{factors}

To help prevent violence victimisation and perpetration, it is crucial to understand the determinants that influence these experiences and target the most vulnerable group. This dissertation responded to this objective by using quantitative measures to examine the sociodemographic correlates (see Chapter 2), as well as the internal and external protective factors (see Chapter 3 ) that are associated with different types of victimisation and perpetration, in a nationally representative sample of South African adolescents and a sample of adolescents from Cape Town, respectively.

\section{Demographic factors}

It was found that age was a predictor: older adolescents were at higher risk for being both a victim and a perpetrator (see Chapter 2), and for witnessing and hearing about violence (see Chapter 3). In addition, Chapter 5 found that age was positively associated with a composite measure of health risk behaviours (smoking, alcohol use, soft drug use, hard drug use, risky sexual behaviour). Together, these results suggest that as the age of adolescents increases, they are exposed to more negative social and peer influences that may also put them at increased risk for violence victimisation and perpetration. For example, engaging in activities such as alcohol and drug use have been associated with violent behaviours (Mitjans et al., 2019), and may put adolescents at further risk for victimisation (Mrug \& Windle, 2009). Furthermore, as the adolescents grow up, they may seek a sense of identity and control to gain "status" among their peers, which can be exacerbated in harsh environments where the struggle for dominance is real. They may seek approval through physical strength (use of violence to gain power over others) and resources (money, goods - acquired by committing robbery). The reward for engaging in violent behaviours and crime is thus salient, and may reinforce young people's tendency to behave as such.

The role of gender was also examined, and our results show that being a boy or a girl is associated with different patterns of risks for victimisation and perpetration. More specifically, being a boy increased the odds of being a perpetrator of violence only and being both a victim and a perpetrator (see Chapter 2). Boys were also at higher risk for delinquent behaviours than girls (see Chapter 3). Both quantitative studies in Chapter $4 \& 5$ found that boys reported more indirect school victimisation through witnessing acts of chasing and beating, and direct community victimisation than girls. On the other hand, being a girl increased the odds of non-involvement and being a victim only (see Chapter 2), and girls reported more indirect home victimisation than boys (see Chapter 5). The findings of this dissertation thus suggest that boys are likely to experience both victimisation and engage in violent behaviours, and that they have more externalising maladjustment characterized by more violent and delinquent behaviours than girls, which is in line with the results in other studies (Begle et al., 2011; Moreira et al., 2008). Moreover, boys and girls are at risk for different types of violence exposure, with boys experiencing victimisation across more developmental contexts than girls. It is possible that due to socialisation of boys, they are more likely than girls to be out on the streets and exposed to the highly available alcohol and drugs in the community (Burton \& Leoshcut, 2013), putting them at higher risk for different experiences of violence.

\section{Internal protective factors}

Both internal and external protective factors may help combat the experiences of victimisation and perpetration, and in this dissertation, we show that such protective factors are uniquely associated with these experiences. In Chapter 3, the results on the internal protective factors showed that self-confidence, social skills, and emotional insight were associated with less peer victimisation. Anger management was associated with less indirect- and peer victimisation. These results highlight that both social and emotional competencies in an adolescent may be important to lower the risk for victimisation, or possibly, to cope with victimisation differently. In particular, self-confidence and social skills are characterised by assertiveness, negotiation, use of humour, and self-control in social settings (Holmes, Yoon, Voith, Kobulsky, \& Steigerwald, 2015; Lepore \& Kliewer, 2019). Moreover, the ability to manage the emotion of anger, appraise negative emotions, and be adept at reading social norms, facial expressions, and intention of others (Holmes et al., 2015) may also help establish and maintain positive relationships with others, and thereby lower the risk for victimisation. 
For the internal protective factors associated with violence perpetration in adolescents, Chapter 3 showed that emotional insight was associated with delinquent behaviours. Anger management, instrumental coping of sadness and anger, and empathy were associated with less peer violence perpetration and less delinquent behaviours. These results suggest that emotional competencies and empathy thus not only play a key role in reducing victimisation, but also in reducing the use of violence in adolescents. It has been established that emotional insight allows adolescents to understand their negative emotions and inspect the situation better, which help them to respond in mindful ways rather than resorting to quick action tendencies (Gartland et al., 2011). Similarly, adolescents who can effectively manage their negative emotions exert better behavioural control and demonstrate effective communication and problem-solving in interpersonal relationships (Kaynak, Lepore, Kliewer, \& Jaggi, 2015; Vanderbilt-Adriance \& Shaw, 2008). Moreover, adolescents who have empathy can understand, appreciate, and tolerate perspectives and emotions of others, thus lower the risk for engaging in violent behaviours that inflict harm (Davis, 2004).

\section{External protective factors}

Since an adolescent is located within a layered ecological system, the interaction with the important stakeholders across different developmental contexts may shape their developmental experiences (Reddy et al., 2011). Studies have found that victimisation experiences in adolescents were lower when there are higher levels of perceived parental care (Scoglio, Kraus, Saczynski, Jooma, \& Molnar, 2019), parental acceptance (Kliewer et al., 2004), and teachers' support and attentiveness (Marachi, Astor, \& Benbenishty, 2007). Similar results have been revealed in this dissertation. In Chapter 2 , adolescents who did not have an absent mother had higher odds of non-involvement in victimisation and perpetration, suggesting that having a positive and functioning mother-child relationship may be helpful to prevent both of these experiences. Furthermore, Chapter 3 found that family- and community connectedness were associated with less indirect victimisation, crime-related victimisation, and peer victimisation. In addition, peer connectedness was associated with less crime-related victimisation, and both peer- and school connectedness were associated with less peer victimisation. These results were further supported by the findings of the qualitative studies in Chapter 6 and 7 where adolescents, parents/caregivers, and teachers believed that rules and consistent monitoring of adults may protect adolescents from being exposed to violence, as the adults are informed and take appropriate action when adolescents may be in danger. Taken together, these results in the dissertation suggest that the support across both proximal and distal developmental contexts (i.e., home, peer, school, and community) may strengthen supervision and monitoring of adolescents, provide safe developmental contexts, and allow them to engage in supportive interpersonal relationships, which protect them from being exposed to violence.
Chapter 3 highlighted that school connectedness was uniquely associated with less peer violence perpetration and delinquent behaviours in adolescents. This result is in line with other studies that teachers' support and attentiveness and a positive school climate may reduce school violence (Lösel \& Farrington, 2012; Marachi, Astor, \& Benbenishty, 2007). School is one of the major developmental contexts where adolescents are present on a regular basis and is a prime site for experiencing safety and participate in established infrastructure and activities that could support violence prevention initiatives (Leoschut \& Kafaar, 2017). Thus, a strengthened commitment to school both socially and academically may be particularly helpful to reduce violent behaviours in adolescents.

\section{Adverse Impact of Victimisation across Multiple Developmental \\ Contexts}

According to the Diathesis-Stress Model (Spielman, Caruso, \& Glovinsky, 1987), negative health outcomes are a result of the interaction between an individual's predispositional vulnerability and an environmental stressor. Given the already stressful developmental period which puts adolescents at risk for psychological and behavioural maladjustment, as well as the high-violence environment in South Africa, adolescents suffer the negative developmental impact of violence exposure.

Chapter 4 and 5 delved deeper into understanding the negative consequences of violence exposure across different developmental contexts on adolescents' psychological functioning and health risk behaviours, respectively, in a representative sample of South African adolescents from the Western Cape Province. The results showed that indirect community victimisation was associated with perceived stress, whereas direct community victimisation was associated with anxiety, depression, hopelessness, and suicide ideation. Indirect political victimisation (witnessing/hearing about incidents of violence and suffering from someone they know) was associated with anxiety and hopelessness. These results align with the studies that established the adverse psychological impact of violence (Barbarin \& Richter, 2001; Kaminer et al., 2013), and highlighted that exposure to political violence can have adverse psychological effects intergenerationally (Kahane-Nissenbaum, 2011).

We also investigated poly-victimisation, and showed that violence across multiple contexts was associated with anxiety, depression, perceived stress, and suicide ideation (see Chapter 4). It has been demonstrated that poly-victimisation may be more detrimental to psychological functioning than comparable amounts of exposure that is concentrated in a single developmental context (Finkelhor, Ormrod, \& Turner, 2007). This puts South African adolescents particularly at risk for psychological problems as poly-victimisation is highly prevalent among this group. As a result, there are relatively few environments 
where an adolescent can feel safe, which can elicit feelings of insecurity and fear (Mrug et al., 2008), and interfere with adaptive coping (Finkelhor et al., 2007).

In addition to the adverse psychological consequences of violence exposure, Chapter 5 confirms an association between violence victimisation and health risk behaivours in South African adolescents. The results described in this chapter indicate that indirect school victimisation was associated with girls' health risk behaviours, and direct community victimisation was associated with both boys' and girls' health risk behaviours. Exposure to violence is associated with heightened psychological distress, and the engagement in health risk behaviours - e.g. alcohol or drug use, risky sexual activities - thus could be a coping mechanism or "an escape" to diminish the negative affect associated with victimisation (Cramer, 2000; Lazarus, 1993). Although it may result in a short-term 'soothing' effect (Tice, Bratslavsky, \& Baumeister, 2001), ultimately this coping method is maladaptive.

Importantly, the mechanism through which violence victimisation affects health risk behaviours may be different for boys and girls. Only for girls, emotion dysregulation (inability to regulate sadness and anger) moderated the associations of indirect home victimisation and direct community victimisation with risk behaviours (see Chapter 5). This suggests that girls who were not able to regulate their negative emotions, engaged in more health risk behaviours when they had high exposure to indirect home victimisation/direct community victimisation compared to those who had low exposure. It is possible that girls are more prone to psychological problems than boys during adolescence (Mrug et al., 2008; Slone \& Mayer, 2015), which makes it challenging for them to regulate negative emotions. As evident in this dissertation, girls reported more anxiety, depression, perceived stress, and suicidal ideation than boys (see Chapter 4), and they reported higher emotion dysregulation than boys (see Chapter 5). Indeed, girls have a tendency to ruminate by having repetitive and recurrent thoughts about a traumatic event and its consequences (Dunn, Gilman, Willett, Slopen, \& Molnar, 2012). Thus, emotion dysregulation poses an important risk factor that interferes with girls' capacities to adaptively cope with violence exposure.

\section{Protective Factors that Enhance Resilience in South African}

\section{Adolescents}

Although exposure to violence has detrimental impact on adolescents' psychological and behaviour functioning, not all adolescents experience these maladjustments (Ward, Martin, Theron, Distiller, 2007; Ostaszewski \& Zimmerman, 2006). These adolescents are able to successfully cope and overcome the negative challenges of victimisation by utilising their individual competencies and the presence of positive environmental influences and support to attain positive developmental outcomes (Harvey, 2007). The very process that leads to adaptive developmental outcomes in the face of adversity is termed "resilience". It constitutes an important area of research to inform interventions that help adolescents at risk for violence victimisation build and obtain personal competences and environmental support that have lasting positive influences on their development and functioning throughout the lifetime.

Chapter 6 and 7 employed a qualitative approach to gain an in-depth understanding of the protective factors that positively enhance resilience in South African adolescents living in high-violence communities, from the perspectives of a group of adolescents and a group of key stakeholders (teachers, school principals, parents/caregivers) in Cape Town, respectively. Both adolescents and stakeholders believed that boundaries and expectations from home and school that are intolerant of violence, social support from people in adolescents' peer network, family, school, and community, and a social norm that promotes prosocial behaviours, may facilitate positive psychological and behavioural adjustment in adolescents. These external protective factors make adolescents feel safe and guided in their developmental environments (Hogue, Liddle, Becker, \& Johnson-Leckrone, 2002), and show them that significant others in their lives are interested and concerned about them (Copeland-Linder, Lambert, \& Lalongo, 2010), which provide a secure foundation for them to establish high levels of self-worth, -efficacy, and -regulation. This adaptive functioning may buffer the stressful and adverse experiences of victimisation and help adolescents positively adjust and engage in developmentally healthy activities (Copeland-Linder et al., 2010; Duggins et al., 2016)

Moreover, this dissertation highlighted that, although they are living in adverse and violence-prone communities, many South African adolescents are aware that they have valuable personal competencies and skills (i.e. internal protective factors) and utilise them to overcome the negative challenges of violence. It was delightful during the interviews with adolescents to hear about their positive attitudes and beliefs, and adaptive and creative strategies, despite the challenging environments they were exposed to. Adolescents' commitment to learning, positive (prosocial) values, positive identity, social competencies, and emotional insight were protective to help maintain positive psychological functioning, keep them away from influences of substance use and violence, and keep them motivated to achieve in life, even if they are exposed to violence (see Chapter 6). These internal protective factors reflect individuals' competencies, skills, and self-perceptions, which play an important role to help adolescents cope with stress and promote adaptive psycho-social functioning (Scales, Benson, Leffert, \& Blyth, 2000).

Importantly, these individual competencies can be learnt and consolidated through education and skills training, at home and in school. As mentioned by the stakeholders, teaching adolescents about violence, positive prosocial values, and adaptive coping strategies such as emotion regulation, and providing them with social and interpersonal skills that refrain them 
from engaging in violence and violence-related activities, are protective for the negative impact of violence and adolescents experience positive adjustment and functioning (see Chapter 7). In Chapter 3, the perceived usefulness of the national curriculum "Life Orientation" in school was associated with less delinquent behaviours in adolescents, which sheds light on the opportunity that effective learning of skills to refrain from violence can positively influence adolescents' behaviours and help them grow up as responsible individuals. Indeed, as adolescents are informed and conscious about positive moral values and have opportunities to learn adaptive coping skills, they may become competent at establishing functional and effective interpersonal relationships, and regulate and control their negative emotions and maladaptive behaviours (Kaynak, Lepore, Kliewer, \& Jaggi, 2015; Vanderbilt-Adriance \& Shaw, 2008; Roberton, Daffern, \& Bucks, 2015). Furthermore, both adolescents and key stakeholders suggested that constructive use of time of adolescents by engaging in positive hobbies, sports, and extra-mural programmes can help relieve the negative emotions aroused from victimisation experiences and motivate adolescents to focus on developmentally healthy activities (see Chapters $6 \& 7$ ).

However, the environmental influences such as adequate social support may not always be available for South African adolescents. This is partly due to the high prevalence of violence exposure across home, school, and community (Kaminer et al., 2013), high rates of single parent households (Meintjes, Hall, Marera, \& Boulle, 2010), parental use of substance and violence (Statistics South Africa, 2016), and violence experienced by South African teachers in school (Woudstra, et al., 2018). These factors each individually and combined limit the available and reliable people in adolescents' major developmental contexts. As shown in Chapter 7, the stakeholders shared the challenges they experienced that compromised their support process for adolescents. These challenges emerged from home, school, and socio-cultural contexts, including dysfunctional home, lack of parenting/support skills, negligence (too busy/afraid to intervene), ineffective school-parent relationship, and the normalisation of violence in South African society. These challenges, or risk factors, allow for the understanding of the process that shape the developmental outcomes in adolescents, and should be considered alongside the protective factors in the development of interventions to enhance the resilience in adolescents at risk for violence exposure.

\section{Strengths and Limitations (Directions for Future Research)}

\section{Strengths}

This dissertation is a needs assessment - an important first step to initiate the development of tailored interventions, and aligns with the national priority of violence prevention in South Africa. It consists of six empirical studies that examined the correlates associated with violence victimisation and violence perpetration, the consequences of violence victimisation, and the factors that may positively enhance resilience to the negative impact of victimisation in South African adolescents.
Mixed-methods were applied in this dissertation, including both quantitative and qualitative approach to provide an in-depth and systematic overview of the problem of violence in South African adolescents. In particular, both adolescents' and their key stakeholders' perspectives were considered to understand youth resilience, providing an integrated view of protective factors.

This dissertation utilises a social ecological framework, considering influences from across adolescents' major developmental contexts (i.e., home, school, community) and sociopolitical influences. Both internal and external factors that are associated with victimisation and perpetration experiences were examined (see Chapters $2 \& 3$ ), and the factors that positively enhance adolescents' resilience were explored across all layers of one's ecological system (see Chapters 6 \& 7).

Multiple types of violence were examined within each study, which enabled a comparative view of different types of victimisation and perpetration. The variables were sophistically differentiated into indirect and direct victimisation (see Chapters 4 \& 5), poly-victimisation (see Chapter 4), different types of victimisation and perpetration (e.g., peer victimisation, delinquent behaviours, see Chapter 3), and victim-perpetrator (see Chapter 2). These measures were adapted to suit the local contexts, and to examine some of the most prevalent types of violence experienced by South African adolescents. This is particularly valuable as refined results can be translated into recommendations for tailored intervention development.

Lastly, the use of nationally representative data in Chapter 2, as well as provincially (Western Cape) representative samples in Chapters $5 \& 6$, helped to provide insight on generalisable data.

\section{Limitations}

Although this dissertation provides an important first step to the understanding of violence among South African adolescents, it is not without opportunities and directions for future research. Secondary data were used for the studies in Chapter 4 and 5 and thus a limited influence in the development of the scales of the questionnaire. There is an opportunity to enhance the psychometric properties of the instrument. For example, indirect home- and school victimisation scales each consisted of two items, measuring two constructs - "beaten" and "chased" in these two settings. This may not be a comprehensive representation of exposure to home and school violence in South African adolescents. Measures for additional types of victimisation such as peer or sibling assault, neglect, and verbal-, physical-, and sexual harassment at home and school settings (Finkelhor et al., 2007) may be strengthened in future research. 
Further, and importantly, since cross-sectional data were used in Chapters 2 to 5, causality between protective factors and violence victimisation and perpetration may not be inferred, and possible bi-directional associations should be taken into consideration. For example, health risk behaviours were hypothesised to be a negative developmental consequence in adolescents exposed to violence (Chapter 5). Although these associations were strongly supported by the literature, a bi-directional relationship is also possible, in the sense that the use of alcohol and drugs may put adolescents at higher risk for violence exposure (Mrug \& Windle, 2009). Longitudinal studies are needed to provide further insight into the mechanism of the associations between violence and its correlates.

Chapters 2 to 6 were based on self-reported data by adolescents. The data collection for this dissertation was conducted ethically and rigorously with the presence and monitoring of the research team throughout the administration of questionnaires. Furthermore, self-reports data are largely suited for a needs assessment to examine experiences from participants' perspectives and tailor the intervention to their experiences (Crutzen \& Göritz, 2010). However, the results may be subjected to self-report bias to some extent. Inflated associations between variables may be possible as adolescents can answer the questionnaire in a way that appears to be self-fulfilling and socially desirable. Conversely, it is possible that the associations we reported are in reality even stronger, because of underreporting. Future research should therefore use varied assessments to obtain a holistic view, including valuing key stakeholders' perspectives (see Chapter 7).

Qualitative approaches were used in Chapter $6 \& 7$ and were able to enhance the understanding of the factors that enhance resilience of adolescents living in high-violence communities, from the perspectives of adolescents and key stakeholders (parents and teachers), respectively. However, participants were recruited from only Cape Town, which can result in selection bias. The views expressed in the interviews may not be representative of all adolescents who are at risk for violence victimisation. In addition, the parent participants were predominantly black and mixed-heritage females from lower socioeconomic settings. Although the findings may not be representative of all South African parents, it provided valuable insights to their experiences and challenges as these parents and their children are the groups that are most affected by violence in South Africa. Future studies may include participants from other provinces to build on and complement the current findings.

Finally, the participants in this dissertation were in-school adolescents. Yet there is a significant proportion of out-of-school adolescents in South Africa and approximately 60\% of first graders will ultimately drop out rather than complete 12th Grade (Department of Basic Education, 2015). Out-of-school youth are more vulnerable to health risk behaviours, poor mental health, delinquency, and violence victimisation, as they do not have the protective factor of the school environment, such as supervision and positive mentoring by teachers and peers (Townsend, Flisher, \& King, 2007). Research may pay attention to this vulnerable population to further complement the understanding of violence in South African adolescents and empower young people.

\section{Practical Implications}

After engaging with the local South African communities, i.e., the adolescents who live in constant fear of violence and crime, the school teachers, and parents/caregivers, it is clear that the current education system is largely dysfunctional and faces enormous challenges. Due to the high prevalence of violence that adolescents encounter across multiple contexts, the learning process is often disturbed, and some adolescents are unable to engage in effective learning. As such, the schools are tasked with having to teach large numbers of learners who experience a range of emotional and behavioural difficulties as a result of their extensive victimisation experiences.

In addition, some teachers reported they had to deal with violent learners. They do not have the skills and time to attend to learners' needs because they are under pressure to deliver quality support for both academic tasks and personal wellbeing of adolescents. These negative influences may largely hamper the quality of education, resulting in that adolescents do not develop the necessary skills and competencies that drive them to become responsible individuals in society. The lack of education and skills may prevent young people from contributing effectively to the country's economy. It may further alienate them, putting them at risk to be stuck in poverty and getting involved with violence-related activities.

Given the lack of social cohesion, the vulnerability of adolescents to violence, hampered quality of teaching in schools, and poor support for teachers, the current South African education curriculum is in need of a transformation by redesigning and reengineering to drive the improvements in the cost, quality, service and speed in the school system (Letseka, 2014). Part of the current national education curriculum is Life Orientation. It consists of lessons where life skills and prosocial behaviours are taught to promote healthy adolescent functioning and adjustment, which is an important opportunity to help learners use their talents to achieve their full physical, intellectual, personal, emotional and social potential (Department of Education, 2011). One of the topics covered in Life Orientation is to build necessary skills and knowledge to refrain from engaging in violent behaviours. In Chapter 3 , learners' perception of the usefulness of Life Orientation with regards to violence prevention was associated with less delinquent behaviours. This sheds light on the notion that "change starts with education" and it is possible that quality education and skills building can help learners learn and appreciate prosocial behaviours, which may ultimately contribute to youth violence reduction in the country. Drawing from the results in the dissertation, it 
is crucial to reform and redesign the existing education system so that it responds actively to young South African's specific socio-cultural contexts, and to include support, training, and monitoring criteria for teaching to enhance education competitiveness in South Africa.

Many South African adolescents reside in high-violence communities. Apart from the important role that school plays in positive adolescent development, this dissertation revealed that community connectedness is a key protective factor that keeps adolescents safe from violence exposure and encourages positive psycho-social functioning. Several urban safety projects in South Africa have been implemented to ensure safety of residents. For example, public and private CCTV, maintaining and securing unused public parks, and participatory safety audits with the local communities to map out crime and safety hotspots and co-create solutions (Urban Safety Reference Group, 2019). However, as evident in this dissertation, many adolescents and their family still live in chronic fear of violence and crime. Going to and back from school is considered as unsafe. Giving the magnitude of violence in South Africa, it is crucial to create a safe developmental contexts and promote a cohesive community for South African adolescents, for example, enhance monitoring and surveillance of the communities, increase positive social activities to keep youth away from the streets, and develop trust among the neighbours and with the police. The local government and the communities should continue to work in partnership to deal with the safety concerns and strengthen social cohesion.

Both the scientific literature and the current dissertation have confirmed that family connectedness make adolescents feel secure and supported, and help them develop competencies to recover from the psychological harm of violence and promote healthy psychological functioning and prosocial behaviours. Family is indeed a safe haven for many young people, however, approximately 3.5 million children in South Africa are orphaned (partly due to the HIV/ AIDS epidemic) (Ellonen \& Salmi, 2011). Children who reside in a single-parent household is significantly associated with both violence victimisation and perpetration in youth (Hong et al., 2017). Parental substance use and intimate partner violence further contribute to disrupted family functioning (Groenewald \& Bhana, 2018). These adverse family situations are evident in this dissertation as some parents are struggling with single parenthood and adolescents do not feel safe and supported at their own homes. It is important to educate parents about the impact of their behaviours on children and enhance parenting skills. Moreover, strengthening family support should not solely be based on the traditional nuclear family structure but adapt to the South African context and involve extended family members or caregivers to maximise the possible support from home.

From a policy perspective, South Africa's National Development Plan envisages that by 2030 "people living in South Africa should feel safe and have no fear of crime" (National Planning Commission, 2012). Reducing and preventing violence is critical to national development and to achieving the United Nations' Sustainable Development Goals. As shown in this dissertation, violence is a normal part in the daily lives in adolescents and the victims of violence are at increased risk for negative psychological and behavioural maladjustment, and violence perpetration. While good professional policing may reduce certain types of crime in South Africa such as armed robberies, organised crime and inter-group conflict (e.g., taxi or gang warfare) (Gould, Mufamadi, Hsiao, \& Amisi, 2017), preventing interpersonal violence that adolescents experience at home, school and community needs a holistic approach. This means targeting the internal and external factors that put them at risk for victimisation to create long-term solutions that address the root causes of violence. It also requires greater investment in socia programmes that address violence and its negative impact on young people. However, while South Africa spends over R126 billion a year on criminal justice, only R9 billion is spent by the state on programmes that can prevent violence and empower the victims of violence (Gould et al., 2017). These programmes are often not targeted for adolescents, rely on a mix of donor and state funding, have overworked and unsupported social and health workers, have lack of data for monitoring and evaluating the effectiveness of these programmes, and lack of scalability and integration into existing government systems (Gould, 2018). To address the problem of violence among South Africa adolescents, there must be a radical shift in how state resources are spent and more investment is needed for targeted youth programmes. In addition, multi-sectoral communication and collaboration must be facilitated among government, academics, NGOs, and the private sector, to co-create solutions on the national agenda of violence reduction.

This dissertation consists of the first step in the Intervention Mapping Framework (Bartholomew et al., 2016). A needs assessment was conducted to understand the problem of violence, the determinants of violence, the consequences of violence, and the determinants that enhance resilience. The results can be used for three intervention objectives to help 1) prevent violence victimisation, 2) prevent violence perpetration, and 3) strengthen resilience in South African adolescents living in high-violence communities. Although some variations are evident in the determinants that are associated with these objectives, they have commonalities. In sum, both individual and environmental factors can influence violence experiences and developmental outcomes. A comprehensive and multi-setting approach for the development of targeted interventions may be promising.

Schools can be the prime site for interventions along with the involvement of family Adolescents typically spend a significant amount of time in school and the adolescents who are at high risk for victimisation and perpetration can be effectively identified, as well as those who are negatively affected by violence exposure. Moreover, the established infrastructure and resources in schools can support violence prevention initiatives and positive coping of adolescents by providing education and skills training. It is importan to enhance personal competencies of individuals, such as self-confidence, empathy, social 
skills, and emotion competencies to reduce the risk for victimisation and perpetration. These skills training can also be used to enhance adaptive coping and help adolescents achieve positive developmental outcomes in the face of violence exposure. Furthermore, it is important to incorporate interpersonal skills training to help forge durable and effective social relationships of adolescents within their social network (home, school, peers, and community), and between key stakeholders (e.g., school-parent relationship). The supportive social network can further be strengthened by building skills in parents/ caregivers and teachers (e.g., effective parenting, counselling skills), so they have the necessary competencies to protect and support adolescents in a high-violence context.

Lastly, transparent and open science and ongoing communications among researchers across the world will further the work in violence among adolescents. It is important to have regular collection of age-, race-, area-, and gender-aggregated data in the prevalence of victimisation and perpetration through national surveillance surveys to continue to understand and target the at-risk adolescents. Moreover, two schools of thoughts are currently evident in the literature (trait-oriented vs. process-oriented approach) for "resilience". A unified measure for complex constructs is encouraged to foster meaningful comparison across studies.

\section{Conclusion}

Violence is one of the ongoing challenges that South African adolescents face. They are at risk for both victimisation and perpetration, which can take a significant toll on their development. This dissertation utilised a mixed-method approach to examine the correlates associated with the experiences and behaviours of violence in South African adolescents from across their ecological system. The results may support the development of programmes that align with the national priority to reduce the risk for victimisation and perpetration in adolescents.

Moreover, the qualitative studies in this dissertation gained an in-depth understanding of the internal and external protective factors, or, developmental assets, that support positive development and functioning in adolescents living in high-violence environment. Importantly, the strength and resilience of South African adolescents should be acknowledged and celebrated. The findings suggest that some adolescents indeed overcome the negative impact of violence exposure by drawing upon their personal competencies and support from trusted adults in their developmental contexts. As such, there are opportunities to strengthen these "developmental assets" that a young person possess and has in their developmental contexts, by developing strengths-based interventions to promote positive developmental outcomes in adolescents living in adverse settings.
In sum, even though this dissertation highlighted that adolescents are negatively affected by the high prevalence of violence in South Africa, they are on a road to recovery - in the sense that protective factors (opportunities) are associated with preventing negative experiences of victimisation and perpetration, and some young people indeed have the developmental assets to overcome the negative impact of violence. To take the empirical work further and to drive the initiative for South Africa to be a safe place for all, a joint effort by means of inter-sectoral and multi-disciplinary collaborations of the key stakeholders are needed. These may include school, family, government, NGOs, private sectors, and scholars to review current practices, policies and implement the change to create an empowered and sustainable future for South African adolescents. 


\section{References}


Aiken, L. S., \& West, S. G. (1991). Multiple regression: Testing and interpreting interactions. Thousand Oaks, CA, US: Sage Publications, Inc.

Aitcheson, R. J., Abu-Bader, S. H., Howell, M. K., Khalil, D., \& Elbedour, S. (2017). Resilience in Palestinian adolescents living in Gaza. Psychological Trauma: Theory, Research, Practice, \& Policy, 9(1), 36.

Albus, K. E., Weist, M. D., \& Perez-Smith, A. M. (2004). Associations between youth risk behaviour and exposure to violence: Implications for the provision of mental health services in urban schools. Behaviour Modification, 28(4), 548-564.

Al-Yagon, M. (2015). Externalising and internalising behaviours among adolescents with learning disabilities: Contribution of adolescents' attachment to mothers and negative affect. Journal of Child \& Family Studies, 24(5), 1343-1357.

Bach, J. M., \& Louw, D. (2010). Depression and exposure to violence among Venda and Northern Sotho adolescents in South Africa: original article. African Journal of Psychiatry, 13(1), 25-35.

Bandura, A. (1977). Self-efficacy: toward a unifying theory of behavioural change. Psychological Review, 84(2), 191

Barbarin, O. A., Richter, L., \& de Wet, T. (2001). Exposure to violence, coping resources, and psychological adjustment of South African children. American Journal of Orthopsychiatry, 71, 16-25.

Bartholomew, L. K., Markham, C. M., Ruiter, R. A. C., Fernàndez, M. E., Kok, G., \& Parcel, G. S. (2016). Planning health promotion programmes: An Intervention Mapping approach (4th ed.). Hoboken, NJ: Wiley.

Beck, A. T., Weissman, A., Lester, D., \& Trexler, L. (1974). The measurement of pessimism: the hopelessness scale. Journal of Consulting and Clinical Psychology, 42(6), 861.

Begle, A. M., Hanson, R. F., Danielson, C. K., McCart, M. R., Ruggiero, K. J., Amstadter, A. B., ,.. \& Kilpatrick, D. G. (2011). Longitudinal pathways of victimisation, substance use, and delinquency: Findings from the National Survey of Adolescents. Addictive Behaviours, 36(7), 682-689.

Bell, C. C., \& McBride, D. F. (2010). Affect regulation and prevention of risky behaviours. Journal of the American Medical Association, 304(5), 565-566.

Bender, P. K., Reinholdt-Dunne, M. L., Esbjorn, B. H., \& Pons, F. (2012). Emotion dysregulation and anxiety in children and adolescents: Gender differences. Personality and Individual Differences, 53(3), 284-288.

Benhorin, S., \& McMahon, S. D. (2008). Exposure to violence and aggression: Protective roles of social support among urban African American youth. Journal of Community Psychology, 36(6), 723-743.

Bernat, D. H., Oakes, J. M., Pettingell, S. L., \& Resnick, M. (2012). Risk and direct protective factors for youth violence: Results from the National Longitudinal Study of Adolescent Health. American Journal of Preventive Medicine, 43(2S1), S57-S66.

Blakely-McClure, S. J., \& Ostrov, J. M. (2016). Relational aggression, victimisation and self-concept: Testing pathways from middle childhood to adolescence. Journal of Youth and Adolescence, 45(2), 376-390.

Bobrowski, K. J., Czabala, J. C., \& Brykczynska, C. (2007). Risk behaviours as a dimension of mental health assessment in adolescents. Archives of Psychiatry and Psychotherapy, 9.

Bombay, A., Matheson, K., \& Anisman, H. (2009). Intergenerational trauma: Convergence of multiple processes among First Nations peoples in Canada. Journal of Aboriginal Health, 5(3), 6-47.

Bonanno, G. A., Galea, S., Bucciarelli, A., \& Vlahov, D. (2007). What predicts psychological resilience after disaster? The role of demographics, resources, and life stress. Journal of Consulting \& Clinical Psychology, 75(5), 671.

Bonanno, R. A. (2007). Bullied to the brink: An investigation of students at risk for depression and suicidal ideation. Dissertation Abstracts International Section A, 68, 1323.

Botticello, A. L. (2009). A multilevel analysis of gender differences in psychological distress over time. Journal of Research on Adolescence, 19(2), 217-247.

Botvin, G. J., Griffin, K. W., \& Nichols, T. D. (2006). Preventing youth violence and delinquency through a universal school-based prevention approach. Prevention Science, 7(4), 403-408.

Boxer, P., Rowell H. L., Dubow, E. F., Landau, S. F., Gvirsman, S. D., Shikaki, K., \& Ginges, J. (2013). Exposure to violence across the social ecosystem and the development of aggression: A test of ecological theory in the IsraeliPalestinian conflict. Child Development, 84(1), 163-177.
Braun, V., \& Clarke, V. (2006). Using thematic analysis in psychology. Qualitative Research in Psychology, 3(2), $77-101$.

Brittian, A. S., \& Humphries, M. L. (2015). Prosocial behaviour during adolescence. In International encyclopedi of the social \& behavioural sciences (Vol. 19, pp. 221-227). Elsevier Oxford.

Bronfenbrenner, U. (1979). The Ecology of Human Development: Experiments by Nature and Design. Cambridge, Massachusetts: Harvard University Press.

Brook, D. W., Rubenstone, E., Zhang, C., Morojele, N. K., \& Brook, J. S. (2011). Environmental stressors, low well-being, smoking, and alcohol use among South African adolescents. Social Science and Medicine, 72(9), 1447-1453.

Brook, J. S., Morojele, N. K., Pahl, K., \& Brook, D. W. (2006). Predictors of drug use among South African adolescents. Journal of Adolescent Health, 38(1), 26-34.

Brown, L. K., Houck, C., Lescano, C., Donenberg, G., Tolou-Shams, M., \& Mello, J. (2012). Affect regulation and HIV risk among youth in therapeutic schools. AIDS and Behaviour, 16(8), 2272-2278.

Brubaker, T. H., Brubaker, E. \& Link, M. (2001). School violence: partnerships with families for school reform. Michigan Family Review, 6(1), 1-11.

Bruwer, B., Govender, R., Bishop, M., Williams, D. R., Stein, D. J., \& Seedat, S. (2014). Association between childhood adversities and long-term suicidality among South Africans from the results of the South African Stress and Health study: a cross-sectional study. BMJ Open, 4(6), e004644.

Burton, P. \& Leoshcut, L. (2013). School Violence in South Africa: Results of the 2012 National School Violence Study. Cape Town: Centre for Justice and Crime Prevention.

Burton, P., \& Leoschut, L. (2013). School violence in South Africa: Results of the 2012 national school violence study. Retrieved from Cape Town, South Afric:

Carlo, G., Knight, G. P., McGinley, M., \& Hayes, R. (2011). The roles of parental inductions, moral emotions, and moral cognitions in prosocial tendencies among Mexican American and European American early adolescents. The Journal of Early Adolescence, 31(6), 757-781.

Carver, C. S. (1997). You want to measure coping but your protocol'too long: Consider the brief cope. International Journal of Behavioural Medicine, 4(1), 92.

Casey, B. J., Jones, R. M., Levita, L., Libby, V., Pattwell, S. S., Ruberry, E. J., et al. (2010). The storm and stress of adolescence: Insights from human imaging and mouse genetics. Developmental Psychobiology, 52, 225-235.

Chen, W. (2010). Exposure to community violence and adolescents' internalising behaviours among African American and Asian American adolescents. Journal of Youth and Adolescence, 39(4), 403-413.

Chmitorz, A., Kunzler, A., Helmreich, I., Tüscher, O., Kalisch, R., Kubiak, T., ... \& Lieb, K. (2018). Intervention studies to foster resilience-A systematic review and proposal for a resilience framework in future intervention studies. Clinical Psychology Review, 59, 78-100.

Choe, D. E., Zimmerman, M. A., \& Devnarain, B. (2012). Youth violence in South Africa: Exposure, attitudes, and resilience in Zulu adolescents. Violence \& Victims, 27(2), 166-181.

Cohen, J., Cohen, P., West, S. G., \& Aiken, L. S. (2003). Applied multiple correlation/regression analysis for the social sciences. Hillsdale, NJ: Erlbaum.

Cohen, S., Kamarck, T., Mermelstein, R. (1983). A global measure of perceived stress. Journal of Health and Social Behaviour, 24, 385-396.

Coleman, C., Wileyto, E. P., Lenhart, C. M., \& Patterson, F. (2014). Multiple health risk behaviours in adolescents: an examination of Youth Risk Behaviour Survey data. American Journal of Health Education, 45(5), 271-277.

Coles, R. L. (2015). Single-father families: A review of the literature. Journal of Family Theory \& Review, 7(2), $144-166$

Compas, B. E., Jaser, S. S., Dunbar, J. P., Watson, K. H., Bettis, A. H., Gruhn, M. A., \& Williams, E. K. (2014) Coping and Emotion Regulation from Childhood to Early Adulthood: Points of Convergence and Divergence. Australian Journal of Psychology, 66(2), 71-81. 
Control, C. f. D. (2014). Conducting Trend Analysis of YRBS data. Retrieved from http://www.cdc.gov/healthyyouth/ $\mathrm{yrbs} / \mathrm{pdf} / \mathrm{yrbs}$ conducting trend analyses.pdf

Cooley, J. L. (2019). The interactive effects of coping strategies and emotion dysregulation on experiences of peer victimisation during middle childhood. Dissertation Abstracts International: Section B: The Sciences and Engineering. ProQuest Information \& Learning. Retrieved from http://login.ezproxy.ub.unimaas.nl/ $\operatorname{login}$ ?url=http://search.ebscohost.com/login.aspx?direct=true\&db=psyh\&AN=2018-58621-094\&site=ehostlive\&scope $=$ site

Cooper, A., \& Ward, C. L. (2012). Intervening with Youth in Gangs. In A. v. d. M. C. L. Ward., A. Dawes (Ed.), Youth violence: sources and solutions (pp. 53-91). Cape Town, South Africa: UCT Press.

Coovadia, H., Jewkes, R., Barron, P., Sanders, D., \& McIntyre, D. (2009). The health and health system of South Africa: Historical roots of current public health challenges. The Lancet, 374(9692), 817-834.

Copeland-Linder, N., Lambert, S. F., \& Ialongo, N. S. (2010). Community violence, protective factors, and adolescent mental health: A profile analysis. Journal of Clinical Child \& Adolescent Psychology, 39(2), 176-186.

Covington, S. S., Burke, C., Keaton, S., \& Norcott, C. (2008). Evaluation of a trauma-informed and genderresponsive intervention for women in drug treatment. Journal of Psychoactive Drugs, 40(5), 387-398.

Cramer, P. (2000). Defense mechanisms in psychology today: Further processes for adaptation. American Psychologist, 55, 637-646.

Croisant, S. P., Haque Laz, T., Rahman, M., \& Berenson, A. B. (2013). Gender differences in risk behaviours among high school youth. Global Advances in Health and Medicine, 2(5), 16-22.

roisant, S. P., Haque Laz, T., Rahman, M., \& Berenson, A. B. (2013). Gender differences in risk behaviours among high school youth. Global Advances in Health and Medicine, 2(5), 16-22.

Crone, E. A., \& Dahl, R. E. (2012). Understanding adolescence as a period of social-affective engagement and goal flexibility. Nature Reviews Neuroscience, 13(9), 636-650.

Crutzen, R., \& Göritz, A. S. (2010). Social desirability and self-reported health risk behaviours in web-based research: three longitudinal studies. BMC Public Health, 10(1), 720

Dada, S., Burnhams, N. H., Erasmus, J., Parry, C., Bhana, A., Timol, F., \& Fourie, D. (2016). Alcohol and drug abuse trends: July-December 2015: Phase 39

Das, J. K., Salam, R. A., Arshad, A., Finkelstein, Y., \& Bhutta, Z. A. (2016). Interventions for Adolescent Substance Abuse: An Overview of Systematic Reviews. Journal of Adolescent Health, 59(4), 61-75.

Das-Munshi, J., Lund, C., Mathews, C., Clark, C., Rothon, C., \& Stansfeld, S. (2016). Mental health inequalities in adolescents growing up in post-apartheid south africa: Cross-sectional survey, SHaW study. PloS one, 11(5),

Davis, M. H. (2006). Empathy. In Handbook of the sociology of emotions (pp. 443-466). Springer, Boston, MA.

Dekel, B., Abrahams, N., \& Andipatin, M. (2018). Exploring adverse parent-child relationships from the perspective of convicted child murderers: A South African qualitative study. PloS one, 13(5), e0196772.

Department of Education (2011). National Curriculum Statement: curriculum and assessment policy Statement. Retrieved from https://www.education.gov.za/Portals/0/CD/National\%20Curriculum\%20Statements\%20and\%20 pdf?ver=2015-01-27-154251-017

Department of Education (2015). Education statistics in South Africa 2013. Pretoria: Department of Basic Education, Republic of South Africa. Retrieved from http://www.education.gov.za/Portals/0/Documents/Publications/ Education\%20Statistic\%202013.pdf?ver=2015-03-30-144732-767.

Du Plessis, A., \& Louw, A. (2005). Crime and Crime Prevention in South Africa: 10 Years After. Canadian Journal of Criminology \& Criminal Justice, 47(2), 427-446.

Dubow, E. F., Boxer, P., Huesmann, L. R., Shikaki, K., Landau, S., Gvirsman, S. D., \& Ginges, J. (2010). Exposure to Conflict and Violence across Contexts: Relations to Adjustment among Palestinian Children. Journal of Clinical Child and Adolescent Psychology, 39(1), 103-116.
Duggins, S. D., Kuperminc, G. P., Henrich, C. C., Smalls-Glover, C., \& Perilla, J. L. (2016). Aggression among adolescent victims of school bullying: Protective roles of family and school connectedness. Psychology of Violence, 6(2), 205.

Duke, A. A., Smith, K. Z., Oberleitner, L. S., Westphal, A., \& McKee, S. A. (2018). Alcohol, drugs, and violence: meta-meta-analysis. Psychology of Violence, 8(2), 238-249.

Dunn, E. C., Gilman, S. E., Willett, J. B., Slopen, N. B., \& Molnar, B. E. (2012). The impact of exposure to interpersonal violence on gender differences in adolescent-onset major depression: results from the National Comorbidity Survey Replication (NCS-R). Depression and Anxiety, 29(5), 392-399.

Duque, L. F., Montoya, N. E., \& Restrepo, A. (2011). Violence witnessing, perpetrating and victimisation in medellin, Colombia: a random population survey. BMC Public Health, 11(1), 628

Dvir, Y., Ford, J. D., Hill, M., \& Frazier, J. A. (2014). Childhood maltreatment, emotional dysregulation, and psychiatric comorbidities. Harvard Review of Psychiatry, 22, 149-161.

Education, D. o. B. (2011). Report on Dropout and Learner Retention Strategy to Portfolio Committee on Education. Retrieved from https://www.education.gov.za/Portals/0/Documents/Reports/REPORT\%20ON\%20 DROPOUT\%20AND\%20ETENTION\%20TO\%20PORTFOLIO\%20COMMITTEE\%20JUNE\%202011. pdf?ver=2015-03-20-120521-617

El Achhab, Y., El Ammari, A., El Kazdouh, H., Najdi, A., Berraho, M., Tachfouti, N., ... \& Nejjari, C. (2016). Health risk behaviours amongst school adolescents: protocol for a mixed methods study. BMC Public Health, 16(1), 1209

Ellis, B. H., Abdi, S. M., Miller, A. B., White, M. T., \& Lincoln, A. K. (2015). Protective factors for violence perpetration in Somali young adults: The role of community belonging and neighborhood cohesion. Psycholog. of Violence, 5(4), 384.

Ellonen, N., \& Salmi, V. (2011). Poly-victimisation as a life condition: Correlates of poly-victimisation among Finnish children. Journal of Scandinavian Studies in Criminology \& Crime Prevention, 12(1), 20-44.

Feeney, J. A., Peterson, C., Gallois, C., \& Terry, D. J. (2000). Attachment style as a piedictor of sexual attitudes an behaviour in late Adolescence. Psychology \& Health, 14(6), 1105-1122.

Fergus, S., \& Zimmerman, M. A. (2005). Adolescent resilience: A framework for understanding healthy developmen in the face of risk. Annual Review of Public Health, 26, $399-419$.

Field, A. (2009). Discovering statistics using SPSS (3rd ed.). Thousand Oaks, CA: SAGE.

Finkelhor, D. (2009). Children's Exposure to Violence: A Comprehensive National Survey. Retrieved from Washington, DC: US Department of Justice, Office of Justice Programmes, Office of Juvenile Justice and Delinquency Prevention.:

Finkelhor, D., Hamby, S. L., Ormrod, R., \& Turner, H. (2005). The Juvenile Victimisation Questionnaire: reliability, validity, and national norms. Child Abuse \& Neglect, 29(4), 383-412.

Finkelhor, D., Ormrod, R. K., \& Turner, H. A. (2007). Poly-victimisation: A neglected component in child victimisation. Child Abuse and Neglect, 31, 7-26.

Finkelhor, D., Ormrod, R. K \& Turner, H. A. (2007). Re-victimisation patterns in a national longitudinal sample of children and youth. Child Abuse \& Neglect, 31(5), 479-502.

Finkelhor, D., Ormrod, R., Turner, H., \& Holt, M. (2009). Pathways to poly-victimisation. Child Maltreatment 14(4), 316-329.

Finkelhor, D., Turner, H., Hamby, S. L., \& Ormrod, R. (2011). Poly-victimisation: Children’s exposure to multiple types of violence, crime, and abuse. Free Inquiry in Creative Sociology, 39(2), 45-63.

Finkelhor, D., Turner, H., Ormrod, R., Hamby, S., \& Kracke, K. (2009). Children's Exposure to Violence: A Comprehensive National Survey. Washington, DC: U.S. Department of Justice, Office of Justice Programmes, Office of Juvenile Justice and Delinquency Prevention.

Flouri, E., \& Mavroveli, S. (2013). Adverse life events and emotional and behavioural problems in adolescence: The role of coping and emotion regulation. Stress and Health, 29(5), 360-368. 
Folkman, S., Lazarus, R. S., Dunkel-Schetter, C., DeLongis, A., \& Gruen, R. J. (1986). Dynamics of a stressful encounter: Cognitive appraisal, coping, and encounter outcomes. Journal of Personality and Social Psychology, 50, 992-1003.

Fowler, P. J., Tompsett, C. J., Braciszewski, J. M., Jacques-Tiura, A. J., \& Baltes, B. B. (2009). Community violence: A meta-analysis on the effect of exposure and mental health outcomes of children and adolescents. Development and Psychopathology, 21(01), 227-259.

Fox, C. L., \& Boulton, M. J. (2005). The social skills problems of victims of bullying: Self, peer and teacher perceptions. British Journal of Educational Psychology, 75(2), 313-328.

Garcia-Moreno, C., Pallitto, C., Devries, K., Stockl, H., Watts, C., \& Abrahams, N. (2013). Global and regional estimates of violence against women: prevalence and health effects of intimate partner violence and non-partner sexual violence. Geneve: World Health Organization.

Garner, P. W., \& Hinton, T. S. (2010). Emotional display rules and emotion self-regulation: Associations with bullying and victimisation in community- based after school programmes. Journal of Community \& Applied Social Psychology, 20(6), 480-496.

Gartland, D., Bond, L., Olsson, C. A., Buzwell, S., \& Sawyer, S. M. (2011). Development of a multi-dimensional measure of resilience in adolescents: the Adolescent Resilience Questionnaire. BMC Medical Research Methodology, 11(1), 134.

Georgiou, S. N., \& Stavrinides, P. (2008). Bullies, victims and bully-victims: Psychosocial profiles and attribution styles. School Psychology International, 29(5), 574-589.

Gersh, E., Lee, C. M., \& McCarty, C. A. (2019). Changes in peer norms as a mediator of reduction in adolescent alcohol use. Substance Use \& Misuse, 54(10), 1611-1617.

Gevers, A., \& Flisher, A. (2012). School-based youth violence prevention interventions. In C. Ward, A. van der Merwe, \& A. Dawes (Eds.), Youth violence: sources and solutions in South Africa (pp. 175-121). Cape Town, South Africa: UCT Press.

Gini, G., Albiero, P., Benelli, B., \& Altoe, G. (2007). Does empathy predict adolescents' bullying and defending behaviour?. Aggressive Behaviour: Official Journal of the International Society for Research on Aggression, 33(5), $467-476$.

Goldstein, A. L., Vilhena-Churchill, N., Stewart, S. H., \& Wekerle, C. (2012). Coping motives as moderators of the relationship between emotional distress and alcohol problems in a sample of adolescents involved with child welfare. Advances in Mental Health, 11(1), 67-75.

Gould, C. (2018). Glimmers of Light: Opportunities for Preventing Violence in South Africa. Retrieved from https:// issafrica.s3.amazonaws.com/site/uploads/2018-05-wilson-research-paper-21.pdf.

Gould, C., Mufamadi, D., Hsiao, C., \& Amisi, M. (2017). Reducing violence in South Africa: from policing to prevention. Policy brief.

Griffin, K., Botvin, G., \& Nichols, T. (2006). Effects of a School-Based Drug Abuse Prevention Programme for Adolescents on HIV Risk Behaviour in Young Adulthood. Prevention Science, 7(1), 103-112.

Groenewald, C., \& Bhana, A. (2018). Substance abuse and the family: An examination of the South African policy context. Drugs: Education, Prevention and Policy, 25(2), 148-155.

Gustafsson, P. E., Nilsson, D., \& Svedin, C. G. (2009). Polytraumatization and psychological symptoms in children and adolescents. European Child and Adolescent Psychiatry, 18(5), 274-283.

Guttmann-Steinmetz, S., Shoshani, A., Farhan, K., Aliman, M., \& Hirschberger, G. (2012). Living in the crossfire: Effects of exposure to political violence on Palestinian and Israeli mothers and children. International Journal of Behavioural Development, 36(1), 71-78.

Hague, A., Liddle, H. A., Becker, D., \& Johnson-leckrone, J. (2002). Family-based prevention counseling for highrisk young adolescents: Immediate outcomes. Journal of Community Psychology, 30(1), 1-22.

Hair, J. F., Black, W. C., Babin, B. J., Anderson, R. E., \& Tatham, R. L. (2006). Multivariate Data Analysis. Upper Saddle River, NJ: Pearson Prentice Hall.

Halperin, E., \& Pliskin, R. (2015). Emotions and emotion regulation in intractable conflict: Studying emotional processes within a unique context. Political Psychology, 36, 119-150.
Hamlin, D , \& Li, A (2019). The Relationship between Parent Volunteering in School and School Safety in Disadvantaged Urban Neighborhoods. Journal of School Violence, 1-15.

Hammig, B., \& Jozkowski, K. (2013). Academic achievement, violent victimisation, and bullying among U.S. High school students. Journal of Interpersonal Violence, 28(7), 1424-1436.

Harper, C. C., \& McLanahan, S. S. (2004). Father absence and youth incarceration. Journal of Research on Adolescence, 14(3), 369-397.

Harrison, P. A., Fulkerson, J. A., \& Beebe, T. J. (1997). Multiple substance use among adolescent physical and sexual abuse victims. Child Abuse \& Neglect, 21(6), 529-539.

Harter, S. (2006). The self. In N. Eisenberg, W. Damon, \& R. Lerner (Eds.), Handbook of child psychology. Socia emotional and personality development (Vol. 3, pp. 505-570). New Jersey: Wiley.

Harvey, M. R. (2007). Towards an ecological understanding of resilience in trauma survivors: Implications for theory, research, and practice. Journal of Aggression, Maltreatment \& Trauma, 14(1-2), 9-32.

Haynie, D. L., Farhat, T., Brooks-Russell, A., Wang, J., Barbieri, B., \& Iannotti, R. J. (2013). Dating violence perpetration and victimisation among US Adolescents: Prevalence, patterns, and associations with health complaints and substance use. Journal of Adolescent Health, 53(2), 194-201.

Heinze, J. E., Stoddard, S. A., Aiyer, S. M., Eisman, A. B., \& Zimmerman, M. A. (2017). Exposure to Violence during Adolescence as a Predictor of Perceived Stress Trajectories in Emerging Adulthood. Journal of Applied Developmental Psychology, 49, 31-38.

Henry, D. B., Tolan, P. H., Gorman-Smith, D., \& Schoeny, M. E. (2012). Risk and direct protective factors for youth violence: results from the centers for disease control and prevention's multisite violence prevention project. American Journal of Preventive Medicine, 43(2S1), S67-S75,

Herrenkohl, T. I., Aisenberg, E., Williams, J. H., \& Jenson, J. M. (Eds.). (2011). Violence in context: Curren Evidence on

Herrenkohl, T. I., Huang, B., Tajima, E. A., \& Whitney, S. D. (2003). Examining the link between child abuse an youth violence: An analysis of mediating mechanisms. Journal of Interpersonal Violence, 18(10), 1189-1208

Hessler, D. M. \& Katz, L. F. (2010). Associations between emotional competence and adolescent risky behaviour Journal of Adolescence, 33, 241-246.

Hessler, D. M. (2008). Family stressors, emotional competence, and adolescent risky behaviour. University of Washington.

Hilt, L. M., Cha, C. B., \& Nolen-Hoeksema, S. (2008). Nonsuicidal self-injury in young adolescent girls: moderator of the distress-function relationship. Journal of Consulting and Clinical Psychology, 76(1), 63-71.

Hindin, M. J., \& Gultiano, S. (2006). Associations between witnessing parental domestic violence and experiencing depressive symptoms in Filipino adolescents. American Journal of Public Health, 96(4), 660-663.

Hinsberger, M., Sommer, J., Kaminer, D., Holtzhausen, L., Weierstall, R., Seedat, S., ... \& Elbert, T. (2016) Perpetuating the cycle of violence in South African low-income communities: attraction to violence in young men exposed to continuous threat. European Journal of Psychotraumatology, 7(1), 29099.

Hogue, A., Liddle, H. A., Becker, D., \& Johnson-Leckrone, J. (2002). Family-based prevention counseling for highrisk young adolescents: Immediate outcomes. Journal of Community Psychology, 30(1), 1-22.

Holmes, M. R., Yoon, S., Voith, L. A., Kobulsky, J. M., \& Steigerwald, S. (2015). Resilience in physically abused children: protective factors for aggression. Behavioural Sciences, 5(2), 176-189.

Hong, J. S., Kim, D. H., \& Hunter, S. C. (2017). Applying the social-ecological framework to explore bully-victim subgroups in South Korean schools. Psychology of Violence. doi:10.1037/vio0000132

Horwitz, A. G., Hill, R. M., \& King, C. A. (2011). Specific Coping Behaviours in Relation to Adolescent Depression and Suicidal Ideation. Journal of Adolescence, 34(5), 1077-1085.

Houck, C. D., Barker, D. H., Hadley, W., Brown, L. K., Lansing, A., Almy, B., \& Hancock, E. (2016). The 1-year impact of an emotion regulation intervention on early adolescent health risk behaviours. Health Psycholog. $35(9), 1036-1045$ 
Howard, K., Martin, A., Berlin, L. J., \& Brooks-Gunn, J. (2011). Early mother-child separation, parenting, and child well-being in Early Head Start families. Attachment \& Human Development, 13(1), 5-26.

Jacobs, A. (2011). Life Orientation as experienced by learners: a qualitative study in North-West Province. South African Journal of Education, 31(2), 212-223.

Jain, S., \& Cohen, A. K. (2013). Behavioural adaptation among youth exposed to community violence: A longitudinal multidisciplinary study of family, peer and neighborhood-level protective factors. Prevention Science, 14(6), 606617.

Jain, S., Buka, S. L., Subramanian, S. V., \& Molnar, B. E. (2012). Protective factors for youth exposed to violence: Role of developmental assets in building emotional resilience. Youth Violence and Juvenile Justice, 10(1), 107-129.

Jeon, H. J., M. McCartney, C., Richard, V., \& Johnson, S. J. (2020). Associations between parent-teacher and teacherchild relationships and children's socioemotional functioning. Early Child Development and Care, 1-15.

Jeong, S., Davis, J., \& Han, Y. (2015). Who becomes more violent among Korean adolescents? Consequences of victimisation in school. Criminal Behaviour and Mental Health, 25(2), 141-155.

Kadra, G., Dean, K., Hotopf, M., \& Hatch, S. L. (2014). Investigating Exposure to Violence and Mental Health in a Diverse Urban Community Sample: Data from the South East London Community Health (SELCoH) Survey. PLoS ONE 9(4), e93660.

Kahane-Nissenbaum, M. C. (2011). Exploring intergenerational transmission of trauma in third generation Holocaust survivors (Doctoral thesis). University of Pennsylvania, USA.

Kaminer, D., du Plessis, B., Hardy, A., \& Benjamin, A. (2013). Exposure to violence across multiple sites among young South African adolescents. Peace and Conflict: Journal of Peace Psychology, 19(2), 112.

Kaminer, D., Hardy, A., Heath, K., Mosdell, J., \& Bawa, U. (2013). Gender patterns in the contribution of different types of violence to posttraumatic stress symptoms among South African urban youth. Child Abuse \& Neglect, $37(5), 320-330$.

Kann, L., Kinchen, S., Shanklin, S. L., Flint, K. H., Hawkins, J., Harris, W. A., ... \& Whittle, L. (2014). Youth Risk Behaviour Surveillance - United States, 2013.

Kann, L., McManus, T., Harris, W. A., Shanklin, S. L., Flint, K. H., Hawkins, J., \& ... Zaza, S. (2016). Youth Risk Behaviour Surveillance - United States, 2015. Morbidity and Mortality Weekly Report. Surveillance Summaries (Washington, D.C.: 2002), 65(6), 1-174.

Kaukinen, C., Buchanan, L., \& Gover, A. (2015). Child abuse and the experience of violence in college dating relationships: Examining the moderating effect of gender and race. Journal of Family Violence, 30(8), 1079-1092.

Kaynak, Ö., Lepore, S. J., Kliewer, W., \& Jaggi, L. (2015). Peer victimisation and subsequent disruptive behaviour in school: The protective functions of anger regulation coping. Personality and Individual Differences, 73, 1-6.

Keijsers, L., \& Poulin, F. (2013). Developmental changes in parent-child communication throughout adolescence. Developmental Psychology, 49(12), 2301.

Kelly, B. M., Schwartz, D., Gorman, A. H., \& Nakamoto, J. (2008). Violent victimisation in the community and children's subsequent peer rejection: The mediating role of emotion dysregulation. Journal of Abnormal Psychology, $36,175-185$.

Kewalramani, S., \& Singh, M. S. (2017). Relationship between Aggression and Interpersonal Communication. The International Journal of Indian Psychology, 4(3), 91-112.

Kim, J., \& Cicchetti, D. (2010). Longitudinal pathways linking child maltreatment, emotion regulation, peer relations, and psychopathology. Journal of Child Psychology and Psychiatry, 51, 706-716.

Kliewer, W. (2016). Victimisation and Biological Stress Responses in Urban Adolescents: Emotion Regulation as a Moderator. Journal of Youth and Adolescence, 45(9), 1812-1823.

Kliewer, W., Cunningham, J. N., Diehl, R., Parrish, K. A., Walker, J. M., Atiyeh, C., \& ... Mejia, R. (2004). Violence Exposure and Adjustment in Inner-City Youth: Child and Caregiver Emotion Regulation Skill, Caregiver-Child Relationship Quality, and Neighborhood Cohesion as Protective Factor. Journal of Clinical Child and Adolescent Psychology, 33(3), 477-487.

Kliewer, W., Pillay, B. J., Swain, K., Rawatlal, N., Borre, A., Naidu, T., ... \& Drazdowski, T. K. (2017). Cumulative risk, emotion dysregulation, and adjustment in South African youth. Journal of Child \& Family Studies, 26(7), $1768-1779$
Krug, E., Dahlberg, L., Mercy, J., Zwi, A., \& Lozano, R. (2002). World report on violence and health. Retrieved https://apps. who.int/iris/bitstream/handle/10665/42495/9241545615 eng.pdf;jsessionid=63EF4 099949C85B3DE972C5B66FA375A? sequence $=1$

Latsch, D. C., Nett, J. C., \& Humbelin, O. (2017). Poly-victimisation and its relationship with emotional and socia adjustment in adolescence: Evidence from a national survey in Switzerland. Psychology of Violence, 7(1), 1.

Lazarus, R. S. (1993). Coping theory and research: Past, present, and future. Psychosomatic Medicine, 55, 234-247.

Lee, R. (2012). Community Violence Exposure and Adolescent Substance Use: Does Monitoring and Positive Parenting Moderate Risk in Urban Communities? Journal of Community Psychology, 40(4), 406-421.

Leiner, M., Dwivedi, A. K., Villanos, M. T., Singh, N., Blunk, D., \& Peinado, J. (2014). Psychosocial profile of bullies, victims, and bully-victims: a cross-sectional study. Frontiers in Pediatrics, 2, 1

Leoschut, L., \& Kafaar, Z. (2017). The frequency and predictors of poly-victimisation of South African children and the role of schools in its prevention. Psychology, Health, \& Medicine, 22(1), 81-93.

Lepore, S. J., \& Kliewer, W. (2019). Social intelligence attenuates association between peer victimisation and depressive symptoms among adolescents. Psychology of Violence, 9(6), 644.

Leshem, B., Haj-Yahia, M. M., \& Guterman, N. B. (2016). The role of family and teacher support in post-traumatic stress symptoms among Palestinian adolescents exposed to community violence. Journal of Child and Family Studies, 25(2), 488-502.

Letseka, M.

Lincoln, Y. S, \& Guba, E. A. (1985). Naturalistic inquiry. Beverly Hills, CA: Sage.

Liu, W., Mumford, E. A., \& Taylor, B. G. (2018). The relationship between parents' intimate partner victimisation 'Wd youths'

Lochman, J. E., Boxmeyer, C., Powell, N., Qu, L., Wells, K., \& Windle, M. (2009). Dissemination of the Coping Power programme: importance of intensity of counselor training. Journal of Consulting and Clinical Psycholog. 77(3), 397.

Logan-Greene, P. L., Nurius, P. S., Herting, J. R., Walsh, E., \& Thompson, E. A. (2010). Violent victimisation and perpetration: joint and distinctive implications for adolescent development. Victims \& Offenders, 5(4), 329-353.

Lösel, F., \& Farrington, D. P. (2012). Direct protective and buffering protective factors in the development of youth violence. American Journal of Preventive Medicine, 43(2), S8-S23.

Lund, C., Boyce, G., Flisher, A. J., Kafaar, Z., \& Dawes, A. (2009). Scaling up child and adolescent mental health services in South Africa: Human resource requirements and costs. Journal of Child Psychology and Psychiatry. 50(9), 1121-1130

Maas, C., Herrenkohl, T. I., \& Sousa, C. (2008). Review of research on child maltreatment and violence in youth. Trauma, Violence, \& Abuse, 9(1), 56-67.

MacArthur, G. J., Smith, M. C., Melotti, R., Heron, J., Macleod, J., Hickman, M., ... \& Lewis, G. (2012). Patterns of alcohol use and multiple risk behaviour by gender during early and late adolescence: the ALSPAC cohort. Journa of Public Health, 34(suppl_1), i20-i30.

Marachi, R., Astor, R. A., \& Benbenishty, R. (2007). Effects of student participation and teacher support on victimisation in Israeli schools: An examination of gender, culture, and school type. Journal of Youth and Adolescence, 36(2), 225-240

Massarwi, A. A. (2017). The correlation between exposure to neighborhood violence and perpetration of moderate physical violence among Arab-Palestinian youth: Can it be moderated by parent-child support and gender? The American Journal Of Orthopsychiatry, 87(4), 452-462.

Masten, A. S. \& Coatsworth, J. D. (1998). The development of competence in favorable and unfavorable environments. American Psychologist, 53(2), 205-220

Masten, A. S. (2011). Resilience in children threatened by extreme adversity: Frameworks for research, practice, and translational synergy. Development \& Psychopathology, 23(2), 493-506. 
Matzopoulos, R. G., Thompson, M. L., \& Myers, J. E. (2014). Firearm and nonfirearm homicide in 5 South African cities: A retrospective population-based study. American Journal of Public Health, 104(3), 455-460.

McDonald, C. C., Deatrick, J. A., Kassam-Adams, N., \& Richmond, T. S. (2011). Community violence exposure and positive youth development in urban youth. Journal of Community Health, 36(6), 925.

McKinnon, B., Gariépy, G., Sentenac, M., \& Elgar, F. J. (2016). Adolescent suicidal behaviours in 32 low- and middle-income countries. Bulletin of the World Health Organization, 94(5), 340-350F

McKnight, L. R., \& Loper, A. B. (2002). The effect of risk and resilience factors on the prediction of delinquency in adolescent girls. School Psychology International, 23(2), 186-198.

McLaughlin, K. A., Hatzenbuehler, M. L., \& Hilt, L. M. (2009). Emotion dysregulation as a mechanism linking peer victimisation to internalising symptoms in adolescents. Journal of Consulting and Clinical Psychology, 77(5), 894-904.

McWey, L. M., Claridge, A. M., Wojciak, A. S., \& Lettenberger, K. C. G. (2015). Parent-adolescent relationship quality as an intervening variable on adolescent outcomes among families at risk: Dyadic analyses. Family Relations: An Interdisciplinary Journal of Applied Family Studies, 64(2), 249-262.

Meintjes, H., Hall, K., Marera, D. H., \& Boulle, A. (2010). Orphans of the AIDS epidemic? The extent, nature and circumstances of child-headed households in South Africa. AIDS Care, 22(1), 40-49.

Mennis, J., \& Mason, M. J. (2012). Social and geographic contexts of adolescent substance use: The moderating effects of age and gender. Social Networks, 34(1), 150-157.

Mitjans, M., Seidel, J., Begemann, M., Bockhop, F., Moya-Higueras, J., Bansal, V., ... \& Duvar, O. (2019). Violent aggression predicted by multiple pre-adult environmental hits. Molecular psychiatry, 24(10), 1549-1564.

Moffitt, T. E., Arseneault, L., Baucom, D., Caspi, A., Chen, E., Danese, A., ... Kuper-Yamanaka, M. (2013). Childhood exposure to violence and lifelong health: Clinical intervention science and stress biology research join forces. Development and Psychopathology, 25(4), 1619-1634.

Morales, J. R., \& Guerra, N. G. (2006). Effects of multiple context and cumulative stress on urban children's adjustment in elementary school. Child Development, 77(4), 907-923.

Morantz, G., Cole, D., Vreeman, R., Ayaya, S., Ayuku, D., \& Braitstein, P. (2013). Child abuse and neglect among orphaned children and youth living in extended families in sub-Saharan Africa: What have we learned from qualitative inquiry? Vulnerable Children \& Youth Studies, 8(4), 338-352.

Moreira, T. C., Belmonte, E. L., Vieira, F. R., Noto, A. R., Ferigolo, M., \& Barros, H. M. (2008). Community violence and alcohol abuse among adolescents: a sex comparison. Jornal De Pediatria, 84(3), 244-250.

Morgan, A., \& Ziglio, E. (2007). Revitalising the evidence base for public health: an assets model. Promotion \& Education, 14(2), 17-22.

Morojele, N. K., \& Brook, J. S. (2006). Substance use and multiple victimisation among adolescents in South Africa. Addictive Behaviours, 31(7), 1163-1176

Mrug, S., \& Windle, M. (2009). Bidirectional influences of violence exposure and adjustment in early adolescence: Externalising behaviours and school connectedness. Journal of Abnormal Child Psychology, 37(5), 611-623.

Mrug, S., \& Windle, M. (2009). Initiation of alcohol use in early adolescence: links with exposure to community violence across time. Addictive Behaviours, 34(9), 779-781.

Mrug, S., \& Windle, M. (2010). Prospective effects of violence exposure across multiple contexts on early adolescents internalising and externalising problems. Journal of Child Psychology and Psychiatry, 51(8), 953-961.

Mrug, S., Loosier, P. S., \& Windle, M. (2008). Violence exposure across multiple contexts: Individual and joint effects on adjustment. American Journal of Orthopsychiatry, 78(1), 70-84.

Mrug, S., Madan, A., \& Windle, M. (2016). Emotional desensitization to violence contributes to adolescents' violent behaviour. Journal of Abnormal Child Psychology, 44(1), 75-86.

ational Planning Commission (2012). National Development Plan 2030: Our future - make it work, The Presidency, Pretoria.

Norman, R., Matzopoulos, R., Groenewald, P., \& Bradshaw, D. (2007). The high burden of injuries in South Africa. Bulletin of the World Health Organization, 85(9), 695-702.
Organization, W. H. (2019). Maternal, newborn, child and adolescent health: adolescent health epidemiology. Retrieved from https://www.who.int/maternal child adolescent/epidemiology/adolescence/en/

Ostaszewski, K., \& Zimmerman, M. A. (2006). The effects of cumulative risks and promotive factors on urba adolescent alcohol and other drug use: A longitudinal study of resiliency. American Journal of Communit Psychology, 38(3-4), 251-262.

Østergaard, J., Järvinen, M., \& Andreasen, A. G. (2018). A matter of rules? A longitudinal study of parents' influence on young people's drinking trajectories. European Addiction Research, 24(4), 206-215.

Parker, E. M., \& Bradshaw, C. P. (2015). Teen dating violence victimisation and patterns of substance use among high school students. The Journal of Adolescent Health: Official Publication of The Society for Adolescent Medicine, 57(4), 441-447.

Paus, T., Keshavan, M., \& Giedd, J. N. (2008). Why do many psychiatric disorders emerge during adolescence? Nature Reviews. Neuroscience, 9(12), 947-957.

Pellegrini, A. D., \& Long, J. D. (2002). A longitudinal study of bullying, dominance, and victimisation during the transition from primary school through secondary school. British Journal of Developmental Psychology, 20(2), 259-280.

Pereda, N., Guilera, G., \& Abad, J. (2014). Victimisation and poly-victimisation of Spanish children and youth Results from a community sample. Child Abuse and Neglect, 38(4), 640-649.

Pérez-González, A., Guilera, G., Pereda, N., \& Jarne, A. (2017). Protective factors promoting resilience in the relation between child sexual victimisation and internalising and externalising symptoms. Child Abuse \& Neglec

Pérez-González, A., Pereda, N., Guilera, G., Abad, J., \& Finkelhor, D. (2018). Caregiver victimisation in community adolescents: Protective factors related to resilience. Psychology of Violence, 8(2), 163 ,

Perren, S., Dooley, J, Shaw, T, \& Cross, D. (2010). Bullying in school and cyberspace: Associations with depressive symptoms in Swiss and Australian adolescents. Child and Adolescent Psychiatry and Mental Health, 4(1), 28.

Peters, G. J., \& Crutzen, R. (2018). Establishing determinant relevance using CIBER: an introduction and tutorial. Petersen, I., Swartz, L., Bhana, A., \& Flisher, A. J. (2010). Mental health promotion initiatives for children and youth in contexts of poverty: The case of South Africa. Health Promotion International, 25(3), 331-34

Pillai, A., Andrews, T., \& Patel, V. (2009). Violence, psychological distress and the risk of suicidal behaviour in young people in India. International Journal of Epidemiology, 38(2), 459-469.

Prinsloo, E. (2007). Implementation of life orientation programmes in the new curriculum in South African schools perceptions of principals and life orientation teachers. South African Journal of Education, 27(1), 155-170.

Profe, W., \& Wild, L. G. (2017). Mother, father, and grandparent involvement. Journal of Family Issues, 38(6), 776-797.

Pumariega, A. J., Burakgazi, H., Unlu, A., Prajapati, P., \& Dalkilic, A. (2014). Substance abuse: Risk factors for Turkish youth. Bulletin of Clinical Psychopharmacology, 24(1), 5-14.

Radloff, L. S. (1977). The CES-D scale: A self-report depression scale for research in the general population. Applied Psychological Measurement, 1(3), 385-401.

Raymond, J. M., \& Zolnikov, T. R. (2018). Aids-affected orphans in sub-saharan africa: A scoping review on outcome differences in rural and urban environments. AIDS and Behaviour, 22(10), 3429-3441.

Reddy, S. P., James, S., Sewpaul, R., Koopman, F., Funani, N. I., Sifunda, S., . . Omardien, R. G. (2010). Umthente Uhlaba Usamila - The South African Youth Risk Behaviour Survey 2008. Retrieved from Cape Town: South African Medical Research Council.

Reddy, S. P., James, S., Sewpaul, R., Sifunda, S., Ellahebokus, A., Kambaran, N. S., \& Omardien, R. G. (2013). Umthente uhlaba usamila: The 3rd South African national Youth Risk Behaviour Survey 2011. Retrieved from Cape Town: South African Medical Research Council.

Reddy, S. P., Panday, S., Swart, D., Jinabhai, C. C., Amosun, S. L., James, S., .. . Van den Borne, H. W. (2003). Umthenthe Unlaba Usamila - The South African Youth Risk Behaviour Survey 2002. Retrieved from Cape Town: South African Medical Research Council. 
Reingle, J. M., \& Maldonado-Molina, M. M. (2012). Victimisation and violent offending: An assessment of the victim-offender overlap among Native American adolescents and young adults. International Criminal Justice Review, 22(2), 123-138.

Reyes, H. L. M., Foshee, V. A., Chen, M. S., \& Ennett, S. T. (2017). Patterns of dating violence victimisation and perpetration among Latino youth. Journal of Youth and Adolescence, 46(8), 1727-1742.

Reyna, V. F., \& Farley, F. (2006). Risk and rationality in adolescent decision making: Implications for theory, practice, and public policy. Psychological science in the public interest, 7(1), 1-44.

Reynolds, C. R., \& Richmond, B. O. (1985). Revised children's manifest anxiety scale. Los Angeles: Western Psychological Services.

Richter, L. M., Mathews, S., Kagura, J., \& Nonterah, E. (2018). A longitudinal perspective on violence in the lives of South African children from the Birth to Twenty Plus cohort study in Johannesburg-Soweto. South African Medical Journal, 108(3), 181-186.

Richters, J. E., \& Saltzman, W. (1990). Survey of exposure to community violence: Self-report version. JE Richters.

Roberton, T., Daffern, M., \& Bucks, R. S. (2015). Beyond anger control: Difficulty attending to emotions also predicts aggression in offenders. Psychology of Violence, 5(1), 74.

Rogers, M., Rumley, T., \& Lovatt, G. (2019). The Change Up Project: Using Social Norming Theory with Young People to Address Domestic Abuse and Promote Healthy Relationships. Journal of Family Violence, 34(6), 507519 .

Roy, A., Noormohamed, R., Henderson, R. I., \& Thurston, W. E. (2015). Promising healing practices for interventions addressing intergenerational trauma among Aboriginal youth: A scoping review. First Peoples Child and Family Review, 10(2), 62-81.

Russell, P. L., Nurius, P. S., Herting, J. R., Walsh, E., \& Thompson, E. A. (2010). Violent victimisation and perpetration: Joint and distinctive implications for adolescent development. Victims \& Offenders, 5(4), 329-353.

Saewyc, E. M., \& Chen, W. (2013). To what extent can adolescent suicide attempts be attributed to violence exposure? A population-based study from Western Canada. Canadian Journal of Community Mental Health, 32(1), 79-94.

Scales, P. C., Benson, P. L., Leffert, N., \& Blyth, D. A. (2000). Contribution of developmental assets to the prediction of thriving among adolescents. Applied Developmental Science, 4(1), 27-46.

Scales, P. C., Benson, P. L., Roehlkepartain, E. C., Sesma Jr, A., \& van Dulmen, M. (2006). The role of developmental assets in predicting academic achievement: A longitudinal study. Journal of Adolescence, 29(5), 691-708.

Schiff, M., Pat-Horenczyk, R., Benbenishty, R., Brom, D., Baum, N., \& Astor, R. A. (2012). High school students' posttraumatic symptoms, substance abuse and involvement in violence in the aftermath of war. Social Science and Medicine, 75(7), 1321-1328

Schiff, M., Pat-Horenczyk, R., Ziv, Y., \& Brom, D. (2017). Multiple traumas, maternal depression, mother-child relationship, social support, and young children's behavioural problems. Journal of Interpersonal Violence, 0886260517725738 .

Schmidt, I. D. (2014). Addressing PTSD in low-income victims of intimate partner violence: moving toward a comprehensive intervention. Social Work, 59(3), 253-260.

Schwartz, D., Proctor, L. J., \& Chien, D. H. (2001). The aggressive victim of bullying. Peer Harassment in School: The plight of the Vulnerable and Victimized, 147-174

Scoglio, A. A., Kraus, S. W., Saczynski, J., Jooma, S., \& Molnar, B. E. (2019). Systematic review of risk and protective factors for revictimisation after child sexual abuse. Trauma, Violence, \& Abuse, 1524838018823274

Scott-Sheldon, L. J., Carey, K. B., Elliott, J. C., Garey, L., \& Carey, M. P. (2014). Efficacy of alcohol interventions for first-year college students: A meta-analytic review of randomized controlled trials. Journal of Consulting and Clinical Psychology, 82(2), 177-188.

Search institute (2017). The developmental assets framework. Retrieved from https://www.search-institute.org/ourresearch/development-assets/developmental-assets-framework/

Search institute (2017). The developmental assets framework. Retrieved from https://www.search-institute.org/ourresearch/development-assets/developmental-assets-framework
Seedat, M. Van Niekerk, A., Jewkes, R, Suffla, S. \& Ratele, K. (2009). Violence and injuries in South Africa: prioritising an agenda for prevention. The Lancet, 374(9694), 1011-1022.

Seedat, M., Van Niekerk, A., Suffla, S., \& Ratele, K. (2014). Psychological research and South Africa's violence prevention responses. South African Journal of Psychology, 44(2), 136-144.

Sharkey, P. T., Tirado-Strayer, N., Papachristos, A. V., \& Raver, C. C. (2012). The effect of local violence on children's attention and impulse control. American Journal of Public Health, 102(12), 2287-2293.

Sharma, M. K., \& Marimuthu, P. (2014). Prevalence and psychosocial factors of aggression among youth. Indian Journal of Psychological Medicine, 36(1), 48-53.

Shek, D. L., \& Liang, J. (2015). Risk factors and protective factors in substance abuse in Chinese adolescents in Hong Kong. In T. Y. Lee, D. L. Shek, R. F. Sun, T. Y. Lee, D. L. Shek, R. F. Sun (Eds.), Student well-being in Chinese adolescents in Hong Kong: Theory, intervention and research (pp. 237-253). New York, NY, US: Springer Science + Business Media

Shields, N., Nadasen, K., \& Pierce, L. (2009). A comparison of the effects of witnessing community violence an direct victimisation among children in Cape Town, South Africa. Journal Of Interpersonal Violence, 24(7), 1192 1208

Shields, N., Nadasen, K. \& Pierce, L. (2013). Community violence and psychological distress in South African and US Children. International Perspectives in Psychology: Research, Practice, Consultation, 2(4), 286.

Shochet, I. M., Dadds, M. R., Holland, D., Whitefield, K., Harnett, P. H., \& Osgarby, S. M. (2001). The efficacy of a universal school-based programme to prevent adolescent depression. Journal of Clinical Child Psychology 30(3), 303-315

Slone, M., \& Mayer, Y. (2015). Gender differences in mental health consequences of exposure to political violence among Israeli adolescents. Children and Youth Services Review, 58, 170-178.

Smokowski, P. Mann, E. Reynolds, A, \& Fraser, M. (2004). Childhood risk and resiliency factors and heo adolescent adjustment in inner city minority youth. Children and Youth Services Review, 26, 63-91.

Soler, L., Paretilla, C., Kirchner, T., \& Forns, M. (2012). Effects of poly-victimisation on self-esteem and posttraumatic stress symptoms in Spanish adolescents. European Child and Adolescent Psychiatry, 21(11), 645-653. Song, S. J., Tol, W., \& Jong, J. (2014). Indero: Intergenerational trauma and resilience between Burundian former child soldiers and their children. Family Process, 53(2), 239-251.

South African Police Service (2016). Annual report 2015/2016. Retrieved from https://www.saps.gov.za/about/ stratframework/annual_report/2015_2016/saps_annual_report_2015_2016.pdf

South African Police Service (2016). Crime situation in South Africa. Retrieved from https://www.saps.gov.za resource_centre/publications/statistics/crimestats/2015/crime_stats.php

Souverein, F. A., Ward, C. L., Visser, I., \& Burton, P. (2016). Serious, violent young offenders in South Africa: Are they life-course persistent offenders?. Journal of Interpersonal Violence, 3l(10), 1859-1882.

Spielman, A. J., Caruso, L. S., \& Glovinsky, P. B. (1987). A behavioural perspective on insomnia treatment. Psychiatric Clinics of North America, 10(4), 541-553.

Spijkerman, R., Van den Eijnden, R. M., Overbeek, G., \& Engels, R. E. (2007). The impact of peer and parenta norms and behaviour on adolescent drinking: The role of drinker prototypes. Psychology \& Health, 22(1), 7-29. Statistics South Africa (2016). South Africa Demographic and Health Survey 2016. Key Indicator report. Retrieved from http://www.statssa.gov.za/publications/Report\%2003-00-09/Report\%2003-00-092016.pdf

Statistics South Africa (2019). Crime statistics 2018/19. Retrieved from http://www.statssa.gov.za/?page id $=737 \&$ id $=5=5$

Stein, B. D., Jaycox, L. H., Kataoka, S. H., Wong, M., Tu, W., Elliott, M. N., \& Fink, A. (2003). A mental health intervention for schoolchildren exposed to violence: a randomized controlled trial. Journal of the American Medical Association, 290(5), 603-611.

Steinberg, L. (2007). Risk taking in adolescence: New perspectives from brain and behavioural science. Current Directions in Psychological Science, 16(2), 55-59. 
Sui, X., Massar, K., Kessels, L. T. E., Reddy, P. S., Ruiter, R. A. C., Sanders-Phillips, K. (2018). Violence exposure in South African adolescents: Differential and cumulative effects on psychological functioning. Journal of Interpersonal Violence, 1-27.

Sui, X., Massar, K., Kessels, L. T., Reddy, P. S., Ruiter, R. A., \& Sanders-Phillips, K. (2020). Exposure to violence across multiple contexts and health risk behaviours in South African adolescents: the moderating role of emotion dysregulation. Psychology \& Health, 35(2), 144-162.

Sui, X., Massar, K., Reddy, P. S., \& Ruiter, R. A. (2020). Developmental assets in South African adolescents exposed to violence: A qualitative study on resilience. Manuscript submitted for publication.

Sui, X., Massar, K., Ruiter, R. A., \& Reddy, P. S. (2020). Violence typologies and sociodemographic correlates in South African adolescents: a three-wave cross-sectional study. BMC public health, 20(1), 221.

Sullivan, T. N., Helms, S. W., Kliewer, W., \& Goodman, K. L. (2010). Associations between sadness and anger regulation coping, emotional expression, and physical and relational aggression among urban adolescents. Social Development, 19(1), 30-51.

Swahn, M. H., Gressard, L., Palmier, J. B., Kasirye, R., Lynch, C., \& Yao, H. (2012). Serious Violence Victimisation and Perpetration among Youth Living in the Slums of Kampala, Uganda. The Western Journal of Emergency Medicine, 13(3), 253-259.

Thompson R. A., \& Meyer S. (2007). Socialization of emotion regulation in the family. In Gross J. J. (Ed.) Handbook of emotion regulation (pp. 249-268). New York: Guilford Press.

Tice, D. M., Bratslavsky, E., \& Baumeister, R. F. (2001). Emotional distress regulation takes precedence over impulse control: If you feel bad, do it! Journal of Personality and Social Psychology, 80(1), 53-67.

Townsend, L., Flisher, A. J., \& King, G. (2007). A systematic review of the relationship between high school dropout and substance use. Clinical child and family psychology review, 10(4), 295-317.

Tull, M. T., Bardeen, J. R., DiLillo, D., Messman-Moore, T., \& Gratz, K. L. (2015). A prospective investigation of emotion dysregulation as a moderator of the relation between posttraumatic stress symptoms and substance use severity. Journal of Anxiety Disorders, 29, 52-60.

Turner, H. A., Finkelhor, D., Hamby, S., \& Henly, M. (2017). Victimisation and adversity among children experiencing war-related parental absence or deployment in a nationally representative US sample. Child Abuse \& Neglect, 67, 271-279.

Turner, H. A., Shattuck, A., Finkelhor, D., \& Hamby, S. (2017). Effects of poly-victimisation on adolescent social support, self-concept, and psychological distress. Journal of Interpersonal Violence, 32(5), 755-780.

Urban Safety Reference Group (2019). 2018/19 The state of urban safety in South Africa report. Retrieved from https://www.saferspaces.org.za/resources/entry/the-state-of-urban-safety-in-south-africa

Vahidi, S., Fathi, A. E., Hosseininasab, S., Moghadam, M., \& Kiani, A. (2007). The effect of social skills training on decreasing the aggression of pre-school children. Iranian Journal of Psychiatry, 2(3), 108-114.

van der Merwe, A. \& Dawes, A. (2007). Youth violence: A review of risk factors, causal pathways and effective intervention. Journal of Child and Adolescent Mental Health, 19(2), 95-113.

van der Merwe, A., Dawes, A., \& Ward, C. L. (2012). The Development of Youth Violence: An Ecological Understanding. In C. L. Ward, A. van der Merwe, \& A. Dawes (Eds.), Youth violence: sources and solutions (pp. 53-91). Cape Town, South Africa: UCT Press.

Vanderbilt-Adriance, E., \& Shaw, D. S. (2008). Conceptualizing and re-evaluating resilience across levels of risk, time, and domains of competence. Clinical Child \& Family Psychology Review, 11(1-2), 30

Vayachuta, P., Ratana-Ubol, A., \& Soopanyo, W. (2016). The study of 'out-of-school' children and youth situations for developing a lifelong education model for 'out-of-school' children and youth. SHS Web of Conferences, 26.

Vermeiren, R., Schwab-Stone, M., Deboutte, D., Leckman, P., \& Ruchkin, V. (2003). Violence Exposure and Substance Use in Adolescents: Findings from Three Countries. (n.d). Journal of the American Academy of Child $\&$ Adolescent Psychiatry, 42(10), 1261.

Villodas, M. T., Bagner, D. M., \& Thompson, R. (2018). A step beyond maternal depression and child behaviour problems: The role of mother-child aggression. Journal Of Clinical Child And Adolescent Psychology, 47(4),
634-641.
Voisin, D. R., \& Hong, J. S. (2012). A meditational model linking witnessing intimate partner violence and bullying behaviours and victimisation among youth. Educational Psychology Review, 24(4), 479-498.

Voisin, D. R., Jenkins, E. J., \& Takahashi, L. (2011). Toward a conceptual model linking community violence exposure to HIV-related risk behaviours among adolescents: Directions for research. Journal of Adolescen Health, 49(3), 230-236

Walsh, W. A., Dawson, J., \& Mattingly, M. J. (2010). How are we measuring resilience following childhood maltreatment? Is the research adequate and consistent? What is the impact on research, practice, and policy? Trauma, Violence \& Abuse, 11(1), 27-41.

Wang, X., Lei, L., Yang, J., Gao, L., \& Zhao, F. (2017). Moral disengagement as mediator and moderator of the relation between empathy and aggression among Chinese male juvenile delinquents. Child Psychiatry \& Human Development, 48(2), 316-326.

Ward, C. L., Martin, E., Theron, C., \& Distiller, G. B. (2007). Factors affecting resilience in children exposed to violence. South African Journal of Psychology, 37(1), 165-187.

Whitbeck, L. B., Hoyt, D. R., McMorris, B. J., Chen, X., \& Stubben, J. D. (2001). Perceived discrimination an early substance abuse among American Indian children. Journal of Health and Social Behaviour, 42(4), 405-424.

Whiteside, L. K., Ranney, M. L., Chermack, S. T., Zimmerman, M. A., Cunningham, R. M., \& Walton, M. A. (2013) The overlap of youth violence among aggressive adolescents with past-year alcohol use-A latent class analysis: aggression and victimisation in peer and dating violence in an inner city emergency department sample. Journal of Studies on Alcohol and Drugs, 74(1), 125-135.

Whitson, M. L., Robinson, S., Valkenburg, K. V., \& Jackson, M. (2020). The benefits of an afterschool music programme for low $\square$ income, urban youth: The music haven evaluation project. Journal of Community Psychology, 48(2), 426-436.

Wills, T. A., Simons, J. S., Sussman, S., \& Knight, R. (2016). Emotional self-control and dysregulation: A dualprocess analysis of pathways to externalising/internalising symptomatology and positive well-being in younger adolescents. Drug and Alcohol Dependence, 163, 37-45.

Wills, T. A., Walker, C., Mendoza, D., \& Ainette, M. G. (2006). Behavioural and emotional self-control: relations to substance use in samples of middle and high school students. Psychology of Addictive Behaviours: Journal of the Society of Psychologists in Addictive Behaviours, 20(3), 265-278.

Wilson, H. W., Woods, B. A., Emerson, E., \& Donenberg, G. R. (2012). Patterns of violence exposure and sexual risk in low-income, urban African American girls. Psychology of Violence, 2(2), 194.

World Health Organisation (2015). Preventing youth violence: an overview of the evidence. Retrieved from https://apps.who.int/iris/bitstream/handle/10665/181008/9789241509251_eng. pdf;jsessionid=B0A85DEC7CFCEB6806004E688E3E509F? sequence=1

World Health Organization (2014). Health for the World's adolescents. A second chance in the second decade. Retrieved from http://apps.who.int/adolescent/second-decade/files/1612 MNCAH_HWA_Executive_Summary. pdf.

Woudstra, M. H., van Rensburg, E. J., Visser, M., \& Jordaan, J. (2018). Learner-to-teacher bullying as a potentia factor influencing teachers' mental health. South African Journal of Education, 38(1), 1-10.

Ye, S., Yu, L., \& Li, K. K. (2012). A cross-lagged model of self-esteem and life satisfaction: Gender differences among Chinese university students. Personality and Individual Differences, 52(4), 546-551.

Zeman, J., Shipman, K., \& Penza-Clyve, S. (2001). Development and initial validation of the Children's Sadness Management Scale. Journal of Nonverbal Behaviour, 25(3), 187-205.

Zeman, J., Shipman, K., \& Suveg, C. (2002). Anger and sadness regulation: predictions to internalising an externalising symptoms in children. Journal of Clinical Child and Adolescent Psychology, 31(3), 393-398.

Zimmerman, G. M., \& Posick, C. (2016). Risk factors for and behavioural consequences of direct versus indirect exposure to violence. American Journal of Public Health, 106(1), 178-188.

Zych, I., \& Llorent, V. J. (2019). Affective empathy and moral disengagement related to late adolescent bullying perpetration. Ethics \& Behaviour, 29(7), 547-556. 
Summary

Samenvatting 


\section{Violence in South African adolescents: a road to recovery}

\section{Summary}

South Africa has one of the highest prevalence of violence and crime in the world. Adolescents disproportionally suffer the consequences of violence and some are involved in violence perpetration, contributing to the vicious cycle of violence. In light of the sheer size of the adolescent population in the country and the huge potential they have to drive the country's future economy and development, it is crucial to ensure adolescents are empowered and supported and can grow up safely in their developmental contexts, to acquire the essential skills and competencies to be thriving individuals. This thesis investigated violence in South Africa adolescents, by understanding 1) the correlates associated with vicitmisation and perpetration; 2) the developmental consequences of violence victimistion; and 3) the factors that positively enhance resilience in South African adolescents. Six empirical studies made up this thesis and the major recommendations are summarised below.

Chapter 2 was a quantitative study to examine the associations of sociodemographic correlates with four distinct violence typologies, namely, 1) non-involvement in both victimisation and perpetration, 2) victims only, 3) perpetrators only, and 4) victim-perpetrators, in three combined nationally representative samples of South African adolescents of the survey years of 2002, 2008, and 2011. In addition, the trends in the prevalence of the four violence typologies over the three survey years were examined. The results suggest that the majority of adolescents had experienced victimisation, perpetration, or both. Adolescents who did not have an absent mother at home were more likely to be non-involved in any victimisation and perpetration.

To further understand the factors associated with victimisation and perpetration, Chapter 3 zoomed in to focus on a sample of adolescents from communities in Cape Town, where violence and crime are highly prevalent. This study examined the associations of a range of protective factors with adolescents' experiences of violence victimisation (indirect victimisation, crime-related victimisation, peer/sibling victimisation), and violence perpetration (peer/sibling violence perpetration, delinquent behaviours). The results showed that all adolescents reported to have experienced at least one incident of victimisation. The vast majority (90\%) perpetrated at least one incident of violence. Both internal and external protective factors can help combat the experiences of victimisation and perpetration. Selfconfidence, social skills, anger management, and emotional insight were associated with less victimisation experiences. Anger management, instrumental coping of sadness and anger, empathy, and emotional insight was associated with less perpetration behaviours. Family-, peer, school, and community connectedness were associated with less victimisation experiences. In particular, school connectedness and perceived usefulness of Life Orientation in school were associated with less perpetration experiences.

In the next two chapters, the psychological and behavioral impact of violence exposure were examined. Chapter 4 examined the psychological impact of different types of violence victimisation across major developmental contexts, namely, indirect home-, indirect school-, indirect community-, direct community, and indirect political victimisation in a representative sample of South African adolescents in the Western Cape Province. Both differential (individual types) and cumulative effects of victimisation (poly-victimisation) were investigated. The results showed that indirect community victimisation was associated with perceived stress whereas direct community victimisation was associated with anxiety, depression, hopelessness, and suicide ideation. Indirect political victimisation was associated with anxiety and hopelessness. Poly-victimisation was high in the sample and was associated with anxiety, depression, perceived stress, and suicide ideation.

Chapter 5 examined the association between violence exposure across major developmental contexts and a composite measure of health risk behaviours in a representative sample of South African adolescents in the Western Cape Province, and the moderating role of emotion dysregulation in this association. The role of gender was examined to understand the associations for boys and girls separately. The results showed that boys reported more risk behaviours than girls. Direct community victimisation was associated with boys' risk behaviours. Indirect school victimisation and direct community victimisation were associated with girls' risk behaviours. Girls reported higher emotion dysregulation than boys. Only for girls, emotion dysregulation moderated the associations of indirect home victimisation and direct community victimisation with risk behaviours.

After examining the negative impact of violence on adolescents' development, the next two chapters utilised a qualitative approach to enhance the understanding of the factors that positively influence resilience in South African adolescents in a context of high violence exposure. Chapter 6 explored the developmental assets across different layers in the ecological system that promote positive psychological and behavioural functioning in a group of South African adolescents exposed to violence. Adolescents discussed both internal and external developmental assets that helped them adaptively cope with violence exposure. The internal assets identified in the study entail personal competencies and skills, including commitment to learning, positive values, positive identity, social competencies, and emotional insight. The external assets were boundaries and expectations, constructive use of time, and social support from adolescents' external environment, such as peers, family, school, and community. 
Chapter 7 is a continuation of Chapter 6 , and explored the protective factors that promote resilience in adolescents exposed to violence from the key stakeholders in adolescents' lives, i.e., school staff and parents/caregivers. The stakeholders discussed the strategies they used to promote positive psychological and behavioural functioning in their children/ school learners. They also shared the challenges and barriers that compromised the process to enhance positive development in these adolescents. The findings showed that boundaries and expectations, social support, education and skills building, and constructive use of time can help adolescents attain positive developmental outcomes. Challenges and barriers encountered by the stakeholders included dysfunctional home, lack of parenting/support skills, negligence of teachers, ineffective school-parent relationship, stigmatisation of violent learners, and the socio-cultural norm of violence.

This dissertation concludes with practical implications from its findings. The recommendations include:

1) A transformation of the South African education system is needed to support both the academic and personal development of adolescents, as violence is a reality they often live with.

2) Life Orientation in schools that provides education and skills training with regards to violence prevention offers an opportunity to reduce the risk of adolescents engaging in violent behaviours.

3) Promoting safety in the community and connectedness among the neighbours are needed to keep adolescents safe from violence victimisation and encourage positive psychosocial functioning.

4) Family connectedness by means of strengthened support, supervision, and monitoring of adolescents are key to protect adolescents from violence and ensure positive development. Extended family members may be involved to extend the family support for the adolescents living in single parent households.

5) More focus should be put on the investment in the development of targeted youth programmes, as it is currently scarce, fragmented, with lack of data for monitoring and evaluation. The results of the dissertation may be used to inform educational and policy interventions to reduce the risk of victimisation and perpetration experiences in adolescents and promote positive developmental outcomes.
6) Schools may be the prime site for youth programmes and provide skills training to enhance adolescents' self-confidence, empathy, social skills, emotion competencies, and help them establish positive interpersonal relationships with significant others across major developmental contexts to reduce the risk for victimisation and perpetration, and enhance positive developmental outcomes in the face of violence exposure.

7) Skills training for parents/caregivers and teachers (e.g., effective parenting, counselling skills) is needed so they acquire the necessary competencies to protect and support adolescents in a violence-prone environment. 


\section{Geweld bij Zuid-Afrikaanse adolescenten: een weg naar herstel}

\section{Samenvatting}

Zuid-Afrika heeft een van de hoogste prevalenties van geweld en misdaad ter wereld. Adolescenten lijden onevenredig zwaar onder de gevolgen van geweld en sommigen zijn betrokken bij geweldpleging, wat bijdraagt aan de vicieuze cirkel van geweld. In het licht van de enorme omvang van de adolescentenpopulatie in het land en het enorme potentieel dat ze hebben om de toekomstige economie en ontwikkeling van het land te stimuleren, is het cruciaal om ervoor te zorgen dat adolescenten mondiger worden en ondersteund worden, en veilig kunnen opgroeien in hun ontwikkelingscontext, en om de essentiële vaardigheden en competenties te verwerven, om uiteindelijk op te bloeien als individu. Dit proefschrift onderzocht geweld in Zuid-Afrikaanse adolescenten, door inzicht te krijgen in 1) de correlaties die samenhangen met victimisatie en plegen van geweld; 2) de gevolgen voor de ontwikkeling van de jongeren als gevolg van het slachtoffer te zijn van geweld; en 3) de factoren die de veerkracht van Zuid-Afrikaanse adolescenten positief vergroten. Zes empirische studies vormen samen dit proefschrift en de belangrijkste aanbevelingen worden hieronder samengevat.

Hoofdstuk 2 was een kwantitatieve studie gericht op het onderzoeken van de associaties van socio-demografische variabelen met vier verschillende geweldtypologieën, namelijk 1) niet-betrokkenheid bij zowel slachtofferschap als plegen, 2) alleen slachtoffers, 3) alleen daders, en 4) slachtoffer-daders. Hiervoor zijn drie gecombineerde landelijk representatieve steekproeven van Zuid-Afrikaanse adolescenten uit de onderzoeksjaren 2002, 2008 en 2011 gebruikt. Daarnaast werden de trends in de prevalentie van de vier geweldtypologieën over deze drie jaren onderzocht. De resultaten suggereren dat de meerderheid van de jongeren ervaring had met slachtofferschap, plegen van geweld, of beide. Adolescenten die thuis een moeder hadden, waren vaker niet betrokken bij geweld (slachtofferschap en plegen).

Om verder inzicht in de factoren te krijgen die samenhangen met victimisatie en daderschap, werd in hoofdstuk 3 ingezoomd op een steekproef van adolescenten uit de gemeenschappen in Cape Town, waar geweld en criminaliteit een hoge prevalentie hebben. Deze studie onderzocht de associaties van een reeks beschermende factoren met de ervaringen van adolescenten met slachtoffer zijn van geweld en plegen van geweld. De resultaten toonden aan dat alle adolescenten aangaven minstens één incident van slachtofferschap te hebben meegemaakt. De overgrote meerderheid (90\%) pleegde minstens één geweldsincident. Zowel interne en externe beschermende factoren kunnen helpen bij de bestrijding van de gevolgen van geweld, zowel als slachtoffer en als dader: Zelfvertrouwen, sociale vaardigheden, woedebeheersing en emotioneel inzicht werden geassocieerd met minder slachtoffer ervaringen. Woedebeheersing, instrumenteel omgaan met verdriet en woede, empathie en emotioneel inzicht werd geassocieerd met minder daderschap. Verder werd verbondenheid met familieleden, leeftijdsgenoten, op school en in de gemeenschap geassocieerd met minder slachtofferschap ervaringen. Met name schoolverbondenheid en het waargenomen nut van lessen levensoriëntatie op school vertoonden in deze studie een samenhang met minder geweldpleging.

In de volgende twee hoofdstukken van dit proefschrift (hoofdstuk 4 en 5 ) werden de psychologische en gedragsmatige gevolgen van blootstelling aan geweld onderzocht. Hoofdstuk 4 onderzocht de psychologische impact van verschillende soorten slachtofferschap van geweld onder een representatieve steekproef van ZuidAfrikaanse adolescenten uit de West-Kaap provincie. We richtten ons op belangrijke ontwikkelingscontexten, namelijk indirecte blootstelling thuis, op school, en in de gemeenschap; directe blootstelling in de gemeenschap, en indirecte (multi-generatie) blootstelling aan politiek geweld. Zowel differentiële als cumulatieve effecten (polyslachtofferschap) van blootstelling aan geweld en slachtofferschap werden onderzocht. De resultaten toonden aan dat indirect slachtofferschap in de gemeenschap verband hield met meer ervaren stress, terwijl direct slachtofferschap in de gemeenschap werd geassocieerd met meer angst, depressie, hopeloosheid en zelfmoordgedachten. Indirect politiek slachtofferschap werd geassocieerd met meer angst en hopeloosheid. Er was sprake van een hoge mate van poly-slachtofferschap in de steekproef, wat werd geassocieerd met meer angst, depressie, ervaren stress en zelfmoordgedachten.

Hoofdstuk 5 onderzocht het verband tussen blootstelling aan geweld in de belangrijkste ontwikkelingscontexten en gezondheidsrisico-gedrag in een representatieve steekproef van Zuid-Afrikaanse jongeren in de provincie West-Kaap. Tevens werd de invloed van emotieregulatie opdezerelatie onderzocht. Derolvangeslachtwerdonderzochtom deassociaties voor jongens en meisjes afzonderlijk te begrijpen. De resultaten toonden aan dat jongens meer risicogedrag rapporteerden dan meisjes. Direct slachtofferschap in de gemeenschap werd in verband gebracht met meer risicogedrag van jongens. Indirect slachtofferschap op school en direct slachtofferschap in de gemeenschap werden in verband gebracht met meer risicogedrag van meisjes. Meisjes rapporteerden een hogere emotie dysregulatie dan jongens, en voor meisjes had dit invloed op de associaties tussen risicogedrag en zowel indirect slachtofferschap thuis als direct slachtofferschap in de gemeenschap.

$\mathrm{Na}$ het onderzoeken van de negatieve impact van geweld op de ontwikkeling va adolescenten, werd in de volgende twee hoofdstukken een kwalitatieve benadering gebruikt 
om de factoren te begrijpen die de veerkracht van Zuid-Afrikaanse jongeren in een context van blootstelling aan geweld positief kunnen beïnvloeden. Hoofdstuk 6 onderzocht de ontwikkelingsmogelijkheden in verschillende lagen van het ecologische systeem van ZuidAfrikaans adolescenten, gericht op het identificeren van factoren die positief psychologisch en gedragsmatig functioneren kunnen bevorderen in een context van blootstelling aan geweld. Adolescenten bespraken zowel interne als externe factoren die hen hielpen om adaptief om te gaan met blootstelling aan geweld. De interne factoren omvatten persoonlijke competenties en vaardigheden, waaronder toewijding aan leren, positieve waarden, positieve identiteit, sociale competenties en emotioneel inzicht. De externe factoren waren grenzen en verwachtingen thuis, constructief gebruik van tijd en tot slot sociale steun van de externe omgeving van adolescenten, zoals leeftijdsgenoten, familie, school en gemeenschap.

Hoofdstuk 7 is een voortzetting van hoofdstuk 6, maar richtte zich op de belangrijkste personen in het leven van adolescenten, d.w.z. schoolpersoneel en ouders/verzorgers. De belanghebbenden bespraken de strategieën die zij gebruikten om positief psychologisch en gedragsmatig functioneren bij hun kinderen/leerlingen te bevorderen. Ze deelden ook de uitdagingen en barrières die het proces om de positieve ontwikkeling van deze adolescenten te bevorderen, in gevaar brachten. De bevindingen toonden aan dat volgens deze personen het stellen van grenzen en verwachtingen, sociale steun, goed onderwijs en het opbouwen van vaardigheden en constructief gebruik van tijd de adolescenten kunnen helpen om positieve ontwikkelingsresultaten te bereiken. Uitdagingen en belemmeringen waar de belanghebbenden mee werden geconfronteerd waren onder meer een disfunctioneel thuis, gebrek aan opvoedings-ondersteuningsvaardigheden, nalatigheid van leraren, ineffectieve school-ouderrelatie, stigmatisering van gewelddadige leerlingen en de sociaal-culturele norm van geweld.

Dit proefschrift sluit af met praktische implicaties van de bevindingen, en doet de volgende aanbevelingen:

1) Een transformatie van het Zuid-Afrikaanse onderwijssysteem is nodig om zowel de academische als de persoonlijke ontwikkeling van adolescenten te ondersteunen, aangezien geweld een realiteit is waarmee ze vaak leven.

2) Life Orientation lessen op school biede de mogelijkheid om in te gaan op geweld, en de risico's van slachtofferschap en geweldpleging van jongeren te verminderen.

3) Het bevorderen van de veiligheid in de gemeenschap en verbondenheid tussen de buren zijn nodig omjongeren te beschermen tegen geweld en om het positieve psychosociale functioneren aan te moedigen.
4) Gezinsverbondenheid - gericht op versterkte ondersteuning, supervisie en monitoring - is essentieel om adolescenten te beschermen tegen geweld en om een positieve ontwikkeling te verzekeren. De bredere familiekring kan betrokken worden om de gezinsondersteuning voor de adolescenten in éénoudergezinnen uit te breiden.

5) Er moet meer aandacht worden besteed aan het investeren in de ontwikkeling van gerichte jeugdprogramma's, aangezien deze momenteel schaars en versnipperd zijn en er onvoldoende gegevens zijn voor monitoring en evaluatie. De resultaten van dit proefschrift kunnen worden gebruikt om de ontwikkeling van educatieve- en beleidsinterventies te informeren.

6) Scholenkunnendebelangrijkstelocatiezijnvoorjeugdprogramma'senvaardigheidstrainingen bieden om het zelfvertrouwen, de empathie, de sociale vaardigheden en de emotieregulatiecompetenties van adolescenten te vergroten, en hen te helpen positieve interpersoonlijke relaties aan te gaan met belangrijke anderen in belangrijke ontwikkelingscontexten. Zo kunnen het risico op slachtofferschap en pleging van geweld verminderd worden, en positieve ontwikkelingsresultaten behaald worden

7) Vaardigheidstraining voor ouders/verzorgers en leerkrachten is nodig zodat zij de nodige competenties verwerven om adolescenten in een gewelddadige omgeving te beschermen en te ondersteunen. 


\section{Impact}


This dissertation was conducted with the goal to enhance the understanding of the sociodemographic and psychosocial correlates associated with violence victimisation and violence perpetration in South African adolescents. Moreover, adolescents' strengths and resilience were explored in details to uncover the protective factors that can positively influence their development, despite living in communities where violence is prevalent. The impact of this dissertation is four-fold: 1) to address the gaps in the current literature on youth violence and resilience; 2) to provide directions for the key stakeholders in adolescents' lives and enhance their competence to support adolescents; 3 ) to inform the development of targeted interventions for adolescents; 4) to promote social awareness that South African adolescents are a vulnerable group for violence that deserve urgent attention from the society and policy makers.

This dissertation provided an opportunity to enhance the understanding of violence from multiple angles, including violence victimisation across major developmental contexts, indirect and direct victimisation, poly-victimisation, victim-perpetrators, and different types of victimisation and perpetration (e.g., peer victimisation, delinquent behaviours), and several measures of violence were included in each study to allow for comparisons of the negative influence of violence in different forms on adolescent development. In addition, the findings on the personal competencies and external resources (e.g., adult support) that adolescents possess in this dissertation shed light on the developmental assets that many young people have, despite living in challenging environments. This is an incredibly positive thing as there is an opportunity to further validate and enhance the strengths and skills in South African adolescents so they experience a positive developmental process. In addition, the results of the dissertation may be particularly valuable for researchers, social workers, mental health care practitioners, community leaders, and policy makers to inform the work they do in supporting adolescents who are/at risk to be affected by violence.

The results of the dissertation may also benefit the key stakeholders in adolescents' lives, such as parents/caregivers, and school teachers, as they can draw upon the empirical findings and reflect on their current practices and support structures to help adolescents adaptively cope with violence. Based on the current findings in the dissertation, tailored reports were sent to the principals of the schools who participated in the studies, to support them on their strategies to prevent violence. These reports outlined 1) the types and prevalence of violence victimisation and violence perpetration among learners of the specific school; 2) actionable recommendations for the school to develop strategies to prevent learners from exposing to violence, refrain from engaging in aggressive and delinquent behaviours, and promote healthy development. Some examples of the recommendations on the reports for the schools are listed below:
- To build an anti-bullying climate by responding quickly and consistently to incidents of violence and establish clear rules and expectations that violence is not tolerated.

- To enhance school connectedness, school staff can take part in initiating short personal interactions ( 30 seconds or up to 5 minutes) throughout the school day with learners. These interactions are the "resilience touch points" that aims to connect and build trust with learners and show that the teachers care.

- To build knowledge and skills in learners, such as empathy, emotion regulation, the ability to appreciate (socio-cultural) differences of others, and speak up when treated unfairly. Having extra mural activities in schools can help learners realise their interests, skills, and potential.

- To strengthen school-parent relationship by encouraging open communication with parents and understand each other's expectations with regards to adolescent development. For example, talk with parents about school's strategies in responding to violence and involve parents as stakeholders to inform the development of violence prevention protocols in school. Parents and teachers should work together to paint a positive future for adolescents.

Moreover, the results of the dissertation may inform the development of targeted interventions to reduce violence victimisation and violence perpetration in adolescents, and to enhance their resilience to the negative developmental impact of violence victimisation. The next step would be formulating specific programme outcomes and objectives with school principals, teachers, parents, policymakers, NGO's, and other relevant stakeholders. Matrices of Change can be complied, informed by the individual and environmental determinants found in this dissertation that are associated with violence, in combination with sub-behaviours/performance objectives (e.g., walk with a group when going to school to prevent victimisation, refrain from physical bullying behaviour towards peers). Take "anger management" as an example - This was found as a determinant associated with peer violence perpetration in Chapter 3. One of the change objectives for a programme to reduce violent perpetration could be, "adolescents manage their anger in social situations with peers". Other programme objectives may be formulated based on the programme goals, empirical evidence on the determinants of behaviour change, and theories, before designing the programme theme, scope and sequence, as well as the methods and practical applications to leverage change.

Finally, as part of the motivation to start this $\mathrm{PhD}$ trajectory, the results of the dissertation are aimed to enhance the empirical understanding of violence in South African adolescents and to enhance the awareness not just in academia, but also in the general society where social workers, mental health care practitioners, community leaders, youth advocates, and policy 
makers join in to attend to the importance of building a safe and sustainable future for South African adolescents. It may be helpful to strengthen the partnerships, resources, and funding for youth interventions. For example, an NGO with qualified social workers can partner with South African schools, with the funding support from the Department of Education to support positive development of adolescents and enhance teachers' capacity to attend to adolescents and their own psychological health. This may elevate some stress and burdens from the teachers as they often feel unsupported due to high workload and the demand to handle violent incidents in school adequately. Community campaigns on violence reduction may also be useful to enhance social awareness and promote a culture of anti-violence and crime. According to United Nation's Sustainable Developmental Goal (SDG) 16: "Promote peaceful and inclusive societies for sustainable development, provide access to justice for all and build effective, accountable and inclusive institutions at all levels" is part of the global 2030 agenda to reduce violence and related death rates. Conferences and scientific publications in high impact journals have been used to disseminate the knowledge in this dissertation, to support this important international initiative on SDG, and to participate in the dialogue of creating a sustainable and safe future for adolescents. 
Acknowledgements 
My PhD trajectory is a special journey that I deeply cherish. Throughout this journey, I have met incredible colleagues and friends, who have given me a great deal of support.

I would like to thank Professor Priscilla Reddy. Our story began in 2015 October when I first met you and thereafter worked with you on many exciting and meaningful projects. You are at the forefront of public health and health promotion, and you do as much as you can to conduct scientific research that makes a difference in people's lives. This is what I admire about you and I will continue to do something that matters to others in my career. I thank you for playing an important role in creating new opportunities and experiences in my life that I will never forget.

Prof Rob Ruiter, I appreciate your ongoing support throughout my PhD trajectory. You have been an inspiration and aspired me to always reflect upon how knowledge can be translated for the betterment of society. You provide immense patience, compassion, and intellect to our meetings and always made me feel that I have learnt something new and interesting. Under your supervision, I have developed a strong passion for an evidence-based approach for intervention development and behaviour change. Your "Hey Alicia!" in the mornings when you walked pass my office brightened up the days; you often checked up on me to see how my family and I were doing - all these small things count. Your kindness has not only made a positive impact on me, but also on many other $\mathrm{PhD}$ candidates.

Dr Karlijn Massar, I cannot be more proud and grateful to have you as my supervisor. You have mentioned that you'd like to help me become an independent researcher. When I just started my $\mathrm{PhD}$, it required a lot of your time to guide me at every step of the research. You were patient with me, helped me build confidence in research and writing, motivated me to engage in critical and analytical thinking, and encouraged me to be more and more comfortable with research. These valuable experiences have translated into my person life, where I am not afraid of taking on challenges and always eager to learn and try. You are a good supervisor and also a great mentor, who cared for my well-being and showed me the importance of work-life balance. Moreover, I thank you for helping me explore funding opportunities in the final months so I could complete my thesis without constraints.

My special regards go to the amazing colleagues and friends at FPN. Mariella, thank you for your positivity and kindness. You have been a great support to help me with every small things to ensure I have a smooth and wonderful experience at the department.

Kai, I appreciate your refreshing ideas and suggestions during my presentation for one of the papers and I am grateful for your support when I was in need of funding. Your help meant a lot and thank you for all your positive contributions.
To Nathalie, my conference buddy, we have been through different stages of the $\mathrm{PhD}$ together, including overcoming the nervousness of presenting at a conference. I have witnessed your growth. Nothing will come our way if we believe in ourselves. Astin, I cherish our friendship and your infinite good energy that is offered to everyone around you. It is lovely to have shared the adventure with you in Croatia, which has become one of my best memories. To my previous officemate, Abbas, thanks for being a great motivation and for bringing so much fun and laughers to our office. Mart and Bram, we were the "gym rats" after work. I thank you both for always being so kind, insightful, and interesting to talk to Sylvia, I will not forget our beautiful autumn hike in St Pietersburg. Last but not least, a heartfelt thank you to all the members of the department of Work and Social Psychology, Fred, Trudy, Sandra, Merve, Alicia, Gill, Bona, Tari, Marie, Rosine, Fleurie, Sjir, Phillippe, Phil, Gemma, Sarah, Karen, Ute, Irma, Annika, Margje, Fraukje, Robert, Darta, Nino, and Khalid. It makes me smile to know that we have met. Last but not least, a special thank you to Loes, who has supported and supervised me in the beginning part of my $\mathrm{PhD}$ journey and contributed to my learning curve.

To my closest friends, Angelina and Sarah. You mean so much to me and have brought heaps of joy and resilience to my time in Maastricht. Thank you for being part of my progress and for always motivating me to be a better person.

Sylvester, I am happy that you came into my life. You have brought new insights to my life and have made me more self-aware. You have contributed significantly to my ongoing reflection about myself, what I do, and what my passion is. Thanks for experiencing life in the Netherlands with me and for being my backbone.

Finally, I would like to thank my parents. Being your only child means you worry a lot more about me since I left home in 2008. Without your continuous support and wisdom to help me navigate through life, I would not be the person I am today. With all my heart, I thank you both for taking care of me and for making my dreams come true.

"When you want something, all the universe conspires in helping you to achieve it."

- Paulo Coelh 


\section{Curriculum Vitae}


Xincheng (Alicia) Sui was born on 15 February 1990, in Shanghai, China. She moved to South Africa with her family when she was a teenager, earned a double-major bachelor degree in psychology and sociology from University of Cape Town in 2012, an honours degree (cum laude) in psychology from University of the Western Cape in 2014, and a Masters degree in research psychology from University of the Western Cape in 2015. She is also a certified research psychologist at Health Professions Council of South Africa. After research internship, Xincheng worked as a research scientist at Cape Town municipality as well as Human Sciences Research Council in South Africa on projects related to public health, medical education in South Africa, programme evaluation, and (digital) health intervention development. In 2017, she enrolled as a doctoral candidate at Maastricht University in the Netherlands, under the Faculty of Psychology and Neuroscience. Xincheng's research expertise focuses strongly on risk behaviour change, youth development, intervention mapping, and programme evaluation and monitoring.

\section{Publications in this Dissertation}

Sui, X., Massar, K., Ruiter, R. A., \& Reddy, P. S. (2020). Violence typologies and sociodemographic correlates in South African adolescents: a three-wave cross-sectional study. BMC Public Health, 20(1), 221.

Sui, X., Massar, K., Reddy, P. S., \& Ruiter, R. A. (2020). The associations of internal and external protective factors with violence victimization and violence perpetration in South African adolescents. Manuscript submitted for publication.

Sui, X., Massar, K., Kessels, L. T. E., Reddy, P. S., Ruiter, R. A. C., Sanders-Phillips, K. (2018). Violence exposure in South African adolescents: differential and cumulative effects on psychological functioning. Journal of Interpersonal Violence, 1-27.

Sui, X., Massar, K., Kessels, L. T., Reddy, P. S., Ruiter, R. A., \& Sanders-Phillips, K. (2020). Exposure to violence across multiple contexts and health risk behaviours in South African adolescents: the moderating role of emotion dysregulation. Psychology \& Health, 35(2), $144-162$

Sui, X., Massar, K., Reddy, P. S., \& Ruiter, R. A. (2020). Developmental assets in South African adolescents exposed to violence: a qualitative study on resilience. Manuscript submitted for publication.

Sui, X., Massar, K., Reddy, P. S., \& Ruiter, R. A. (2020). Factors that enhance resilience in South African adolescents living in high-violence communities: a qualitative study on stakeholders' perspectives and their challenges. Manuscript in preparation.

\section{Other Publications}

Massar, K., Bělostíková, P., \& Sui, X. (2020). It's the thought that counts: trait self-control is positively associated with well-being and coping via thought control ability. Current Psychology.

Sui, X., Reddy, P., Nyembezi, A., Naidoo, P., Chalkidou, K., Squires, N., \& Ebrahim, S. (2019). Cuban medical training for South African students: a mixed methods study. BMC Medical Education, 19(1), 216.

Cheung, K. L., Durusu, D., Sui, X., \& de Vries, H. (2019). How recommender systems could support and enhance computer-tailored digital health programs: a scoping review. Digital Health, 5, 2055207618824727

Isaacs, S. A., Roman, N. V., Savahl, S., \& Sui, X. (2018). Adapting and validating the family resilience assessment scale in an Afrikaans rural community in South Africa. Communit Mental Health Journal, 54(1), 73-83.

Isaacs, S. A., Roman, N. V., Savahl, S., \& Sui, X. (2018). Using the RE-AIM framework to identify and describe best practice models in family-based intervention development: a systematic review. Child \& Family Social Work, 23(1), 122-136.

Padmanabhanunni, A., \& Sui, X. (2017). Mental healthcare providers' attitudes towards the adoption of evidence-based practice in the treatment of post-traumatic stress disorder in South Africa. South African Journal of Psychology, 47(2), 198-208.

Roman, N. V., Isaacs, S. A., Davids, C., \& Sui, X. (2016). How well are families doing? A description of family well-being in South Africa. Family Medicine and Community Health, 4(3), 9-18

Sui, X., \& Padmanabhanunni, A. (2016). Vicarious trauma: the psychological impact of working with survivors of trauma for South African psychologists. Journal of Psychology in Africa, 26(2), 127-133. 